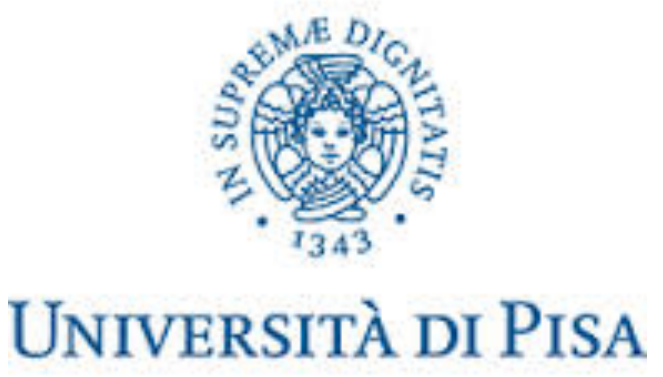

SPECIALIZATION IN OTORHINOLARYNGOLOGY

\title{
Laryngeal preneoplastic lesions and cancer: challenging diagnosis. Qualitative literature review and meta-analysis
}

Author:

Dr. Giuditta Mannelli,
Supervisor:

Prof. Oreste Gallo, 


\begin{abstract}
.
Background: Multi-step cancerogenesis guides laryngeal cancer onset and it includes a wide variety of pre-cancerous lesions that are macroscopically challenging to identify and distinguish from initial cancerous foci. Since the treatment of laryngeal cancer and its precursor lesions has a great impact on important laryngeal basic functions, early detection and preoperative assessment are important for a curative and function-preserving therapy. Nowadays, despite the high number of more advanced diagnostic techniques and methods, unfortunately, it is not uncommon for different clinicians to use different nomenclature or to identify different stage for the same laryngeal lesion.

From these observations, it is obvious that an instrument offering the possibility to detect precancerous lesions, early cancerous lesions, and satellite foci or second primaries would be the key to improving management and outcome in laryngeal patients.
\end{abstract}

Object. Different modalities of diagnostic techniques of laryngeal lesions exist. Rather than difference between benign and obvious malignant diseases, more difficult is to detect the presence of precancerous epithelial alterations. Not all tests achieve the same diagnostic accuracy, hence this meta-analysis of literature aimed to synthesize the validity of each single diagnostic technique in identifying and staging laryngeal disease.

Methods: A systematic review of literature was led searching for articles mentioning the following terms including their various combinations to maximize the yield: larynx, laryngeal precancerous lesions, laryngeal cancer, white light (WL) endoscopy, stroboscopy, contact endoscopy (CE), autofluorescence (AF), ultrasound (US), narrow band imaging (NBI), computed axial tomography (CAT), magnetic resonance imaging (MRI), positron emission tomography (PET), CAT/PET. Then, a quantitative analysis was carried on for paper published after 2005 onward, reporting a minumun series of 10 patients each study, declaring sensitivity and specificity of each diagnostic system.

Results: The search identified 7215 publications, of which 3616 published after 2005, with a final results of a total of 214 articles stratified and included by our selection criteria. 42 out of 214 articles were selected for quantitative synthesis. 25 out of 41 studies had a quality score of $\geq 6$ (good), 16 presented a score between 4 and 5 (fair). While objections can be raised about the pooling of different diagnostic procedures under the same group and the high level of heterogeneity in the meta-analyses, the inclusion of over 4400 laryngeal lesions makes the results fairly robust.

Conclusions: A comprehensive overview of the most recent advances in laryngeal imaging technology combined with all of the information needed to interpret findings and successfully manage patients with voice disorders can be found herein. With these data, clinicians can riskstratify patients and select proper examination modalities in order to provide appropriate care. Moreover, study limitations, together with possible clinical and research implications have been counted, as well.

Key words: laryngeal cancer; laryngeal lesions diagnosis; laryngeal precancerosis; diagnostic imaging; meta-analysis; systematic review 
Table of contest:

1. Introduction.

2. How to write a systematic review and a meta-analysis:

\subsection{Guidelines}

\subsection{Publication bias:}

2.2.1 Risk of bias individuals studies

2.2.2 Meta-bias(es)

2.3 The PRISMA Statement

3. Laryngeal lesions:

3.1 Epithelial precursor lesions:

3.1.1 Definition

3.1.2 Epidemiology

3.1.3 Etiology

3.1.4 Localization

3.1.5 Clinical features

3.1.6 Macroscopy

3.1.7 Histopathology

3.1.8 Differential diagnosis

3.1.9 Prognosis and predictive factors

3.2 Laryngeal cancer

3.2.1 Epidemiology

3.2.2 Incidence

3.2.3 Etiology

4. Laryngeal diagnostic systems and techniques:

\subsection{Endoscopy}

4.2 Stroboscopy

4.3 Contact endoscopy

4.4 Autofluorescence

4.5 Narrow band imaging (NBI)

4.6 Ultrasound

4.7 Computed axial tomography (CAT) and Magnetic resonance imaging (MRI)

4.8 Positron emission tomography (PET, CT/PET)

5. Objectives of the review

6. Methods:

6.1 Study characteristics and quality assessment

6.2 Statistics

7. Results

8. Discussion

9. Limitations

10. Conclusions 


\section{Clinical implications}

\section{Research implications}

13. Disclosure

\section{Conflict of interest statement}

\section{Acknowledgement}

\section{References}

\section{Introduction.}

Across medicine, there has been great effort to parse out the sensitivity and specificity of clinical metrics employed to diagnose disorders related to a given symptom. This principle rests upon the notion that not all tests achieve the same diagnostic accuracy and that all tests must be considered against a gold standard. With these data, clinicians can risk-stratify patients and select proper examination modalities in order to provide appropriate care.

In otolaryngology, hoarseness is the most common laryngeal symptom for which patients seek treatment and may be caused by a diversity of potential disorders. In this setting, visualization of the structure and function of the vocal folds has become an essential component of the clinical voice assessment protocol $(1,2)$, because the capacity of history and physical examination alone to yield adequate information to get the right diagnosis or even risk-stratify patients has not yet been reported. Attempts to examine the human larynx date back more than 150 years, in fact, in 1854 Manuel Garcia, a vocal music teacher, examined his own larynx using a dental mirror and a hand mirror, and published his observations in 1855 (3). Imaging technologies applicable in laryngology developed enormously since then.

Nowadays, the diagnostic procedure of laryngeal diseases in clinical practice is rather complex and is based on the evaluation of patient's complaints, history, and data of instrumental as well as histological examinations. During the last two decades a variety of imaging techniques for the examination of the larynx and obtaining objective measurements of voice quality have been developed (4,5). The term "laryngeal imaging" typically refers to the endoscopic imaging of vocal fold tissue clinical aspect and vibration via videoendoscopy and videostroboscopy; in addition, evaluation of larynx has improved significantly with the establishment of computed axial tomography (CAT) and magnetic resonance imaging (MRI), as these technologies provide insights into the endoscopically blind areas and reveal the depth of tumor infiltration (6). These technologies may be beneficial in staging laryngeal carcinoma and planning the most appropriate surgical procedure $(7,8)$. Recently, ultrasonography has become useful as well in cases of larger laryngeal lesions and may have some role in screening for unilateral vocal fold pathologies. At the same time, further fine-tuning of the technique may be necessary $(9,10)$.

Despite all of these efforts in achieving the perfect diagnostic tool, nowadays surgeons cannot trust one single imaging technique to plan laryngeal patients management and treatment. In this setting, recent reviews emphasized the need for further technological, methodological and clinical research on laryngeal imaging, including the development of clinical norms and objective image processing and measurement methods (11-13). 
This paper provides a review of the latest advances in laryngeal imaging over the last decade in order to give a comprehensive overview of the most recent advances in laryngeal imaging technology combined with all of the information needed to interpret findings and successfully manage patients with voice disorders.

\section{How to write a systematic review and a meta-analysis.}

Systematic reviews and meta-analyses have become increasingly important in health care. Clinicians read them to keep up to date with their field $(14,15)$, and they are often used as a starting point for developing clinical practice guidelines. As with all research, the value of a systematic review depends on what was done, what was found, and the clarity of reporting.

Undertaking a systematic review allows for the acquisition of skills in electronic searching, research methodology, critical appraisal, and statistical analysis. Even when reviews have inconclusive findings, they may be of tremendous value in providing a firm foundation from which to embark on a new research project. Understanding the extent of the evidence base in our specialty and how it is synthesized, greatly empowers the surgeon. This allows the uncertainties of patients and the concerns of health resource managers to be addressed.

High quality Cochrane systematic reviews are undertaken to provide answers to clinical research questions. However, they serve an equally important purpose as a primer for future work. The "empty" review, or one that does not come to a solid conclusion, is often interpreted with disappointment (16), but the review still retains tremendous value. An integral part of every Cochrane review is a section in which the authors comment on "Implications for research." This is an invaluable part of the reviewing process that allows for the constructive synthesis of those failings and criticisms of trials that were not evident until the original trials concluded. It would appear prudent for those undertaking any new project to incorporate these findings before embarking on further study. Unfortunately, many of the methodologic shortcomings are often repeated despite the identification and acknowledgment of these by the authors of previous studies in their conclusions. Paradoxically therefore, a well-conducted and comprehensive systematic review potentially serves as great a purpose as a starting point as it does an endpoint to a clinical question (17).

The literature review lies at the heart of any scientific thesis. Often much of the reviewing work will already have been done by a reviewer woking in collaboration with review groups such as Cochrane group. There are several easily accessed electronic resources in which to search for systematic reviews and meta-analyses: the NLH ENT and Audiology Specialist Library, http:// www.library.nhs.uk/ent/; The Cochrane Library, www.cochrane.org/; PubMed Clinical Queries, www.ncbi.nlm.nih.gov/entrez/query.

As the explosion of scientific knowledge continues, it is difficult to remain up to date by merely reading a few journal publications; moreover, the reporting quality of systematic reviews varies depending on publication bias.

In 1987, Sacks and colleagues (18) evaluated the adequacy of reporting of 83 meta-analyses on 23 characteristics in six domains. Reporting was generally poor; between one and 14 characteristics were adequately reported (mean=7.7; standard deviation=2.7). 
In 1987, Mulrow examined 50 review articles published in four leading medical journals in 1985 and 1986 and found that none met all eight explicit scientific criteria, such as a quality assessment of included studies (19).

In 1996, to address the suboptimal reporting of meta-analyses, an international group developed a guidance called the QUOROM Statement (QUality Of Reporting Of Meta-analyses), which focused on the reporting of meta-analyses of randomized controlled trials (20).

\subsection{Guidelines.}

A systematic review is an overview of primary studies that used explicit and reproducible methods. Systematic reviews apply scientific strategies that limit bias by the systematic assembly, critical appraisal and synthesis of all relevant studies on a specific topic.

It does attempt to collate all relevant evidence that fits pre-specified eligibility criteria to answer a specific research question. It uses explicit, systematic methods to minimize bias in the identification, selection, synthesis, and summary of studies. When done well, this provides reliable findings from which conclusions can be drawn and decisions made.179 180 The key characteristics of a systematic review are: (a) a clearly stated set of objectives with an explicit, reproducible methodology; (b) a systematic search that attempts to identify all studies that would meet the eligibility criteria; (c) an assessment of the validity of the findings of the included studies (such as assessment of risk of bias and confidence in cumulative estimates); and (d) systematic presentation, and synthesis, of the characteristics and findings of the included studies.

A meta-analysis is a mathematical synthesis of the results of two or more primary studies that addressed the same hypothesis in the same way.

Systematic reviews as well as meta-analyses of appropriate studies can be the best form of evidence available for health care practitioners.

A Systematic review protocol is a document that presents an explicit scientific "road map" of a planned, uninitiated systematic review. The protocol details the rational and planned methodological and analytical approach of the review.

SYSTEMATIC REVIEW STEPS:

Research Question

Research Protocol

Literature Search

Data Extraction

Quality Appraisal

Data Analysis and Results

Interpretation of Results

\section{Research Question}

The first step in performing a systematic review is to formulate a primary research question as part of the research protocol. Appropriate questions to be addressed include: (1) phenomena associated 
with disease or interventions, (2) disease or condition frequency, (3) diagnostic accuracy, (4) disease etiology and/or risk factors, (5) prognosis, and (6) intervention effects.

The aims of a systematic review can be varied and include: (1) clarifying the relative strengths and weaknesses of the literature on the question, (2) summarizing a large amount of literature, (3) resolving literature conflicts, (4) evaluating the need for a large clinical trial, (5) avoiding a redundant unnecessary trial, (6) increasing the statistical power of smaller studies, (7) improving the precision or identify a smaller treatment effect, and (8) improving the generalizability of treatment outcomes.

\section{Research Protocol}

A systematic review protocol is important for several reasons: (1) it allows systematic reviewers to plan carefully and thereby anticipate potential problems; (2) it allows reviewers to explicitly document what is planned before they start their review, enabling others to compare the protocol and the completed review (that is, to identify selective reporting), to replicate review methods if desired, and to judge the validity of planned methods; (3) it prevents arbitrary decision making with respect to inclusion criteria and extraction of data; and (4) it may reduce duplication of efforts and enhance collaboration, when available.

Once the research question is formulated, the research protocol is developed. The goal of developing a research protocol is to develop formulation of the questions and methods of the review before retrieving the literature. The methods for literature searching, screening, data extraction, and analysis should be contained in a written document to minimize bias before starting the literature search.

\section{Literature Search}

Sources to search for studies for systematic reviews:

Cochrane Central Register of Controlled Trials (via Cochrane Library)

Cochrane Database of Systematic Reviews (via Cochrane Library)

Database of Abstracts of Reviews of Effects (DARE) database - ie: other reviews (via Cochrane Library)

Medline and Embase (Library does not subscribe to Embase)

Other databases, eg CINAHL, Scopus, PsycINFO

Journals

Conference proceedings

Bibliographies and references listed in primary sources

Unpublished and ongoing studies, including known experts in the field (seek by personal communication)

Raw data from published trials (seek by personal communication)

Foreign language literature (do not limit searches to English)

"Grey literature" (theses, internal reports, non peer-reviewed journals, pharmaceutical industry files) 
PubMed Clinical Queries (www.ncbi.nlm.nih.gov/entrez/query ) includes search filters offering 4 categories of aetiology, prognosis, treatment, diagnosis, and choice of emphasizing sensitivity or specificity.

Cochrane Handbook appendix contains a list of approximately 30 clinical trials registries for finding unpublished primary studies (See Reference List for Library call number)

A minimum of two reviewers performs a first-stage screening of titles and abstracts based on the research question and its study design, population, intervention, and outcome to be studied. Based on the initial screening, selected full-text articles are obtained for the second-stage screening. Using the full text a second-stage screening is performed by at least two reviewers. The studies selected are then submitted for data extraction.

\section{Data Extraction}

A standardized form (paper or electronic) assists in the task of data extraction. For example:

Sample Data Extraction Form Items:

- Reference-including journal, title, author, volume in page numbers Objective - the study objective as stated by the authors

- Study design - type of trial

- Population-demographics of the participants in the study Intervention-description of the intervention

- Control-description of the control group or alternative intervention Outcome - results of the intervention and how measured including statistics used Comments - details regarding the study quality

\section{Quality Appraisal}

A checklist to assess for biases is important: several quality scales and checklists have been developed for this.

Each trial should be evaluated in terms of its:

- Methodological quality - the extent to which the design and conduct are likely to have prevented systematic errors (bias)

- Precision - a measure of the likelihood of random errors (usually depicted as the width of the confidence interval around the result)

- External validity - the extent to which the results are generalizable or applicable to a particular target population

\section{Data Analysis and Results}

After including and excluding studies based on the quality appraisal, data analysis and results of the studies should be undertaken. The initial step for this process involves a simple descriptive evaluation of each study, commonly presented in tabular format. Tables should include the population under study, the interventions, and outcomes. 


\section{Interpretation of Results}

Most of this information can be presented in the data analysis and results table in the manuscript. The strengths and weaknesses of the included studies must be discussed. Conclusions should be based on the best available scientific evidence. Recommendations regarding future studies can also be made.

\subsection{Publication bias.}

As well as positive outcome studies being preferentially published or followed by full text publication (21) authors are more likely to publish positive results in English- language journals (22). This unequal distribution of trials leads to a selection bias in evidence 1 level studies, like systematic reviews, meta-analysis or health technology assessments followed by a systematic failure of interpretation and in clinical decisions.

Even if a study is published in electronic journals not every journal is listed on the major databases like PUBMED.GOV or EMBASE which hinders its identification.

Most of the published studies are not accessible openly; moreover, many large clinical centers do not have all the necessary licenses for all the relevant publications which limit the number of studies they can identify.

Publication bias in a systematic review occurs mostly during the selection process and a transparent selection process is necessary to avoid such bias.

For studies included in a systematic review, a thorough assessment of the risk of bias requires both a "study-level" assessment (e.g., adequacy of allocation concealment) and, for some features, a newer approach called "outcome-level" assessment. An outcome-level assessment involves evaluating the reliability and validity of the data for each important outcome by determining the methods used to assess them in each individual study (23). The quality of evidence may differ across outcomes, even within a study, such as between a primary efficacy outcome, which is likely to be very carefully and systematically measured, and the assessment of serious harms (24), which may rely on spontaneous reports by investigators. This information should be reported to allow an explicit assessment of the extent to which an estimate of effect is correct (23).

Different types of reporting biases may hamper the conduct and interpretation of systematic reviews. Selective reporting of complete studies (e.g., publication bias) (25) as well as the more recently empirically demonstrated "outcome reporting bias" within individual studies (26) should be considered by authors when conducting a systematic review and reporting its results. Though the implications of these biases on the conduct and reporting of systematic reviews themselves are unclear, some previous research has identified that selective outcome reporting may occur also in the context of systematic reviews (27).

\subsubsection{Risk of bias individuals studies.}

An assessment of the risk of bias (or "quality") of studies included in a review is an important component of any well planned or conducted systematic review. Such an assessment contributes to 
the evaluation of the overall strength of evidence of the review. Established methods for assessing risk of bias in reviews have been documented $(28,29)$.

Descriptions of the planned approach to assessing risk of bias should include the constructs being assessed and a definition for each, reviewer judgment options (high, low, unclear), the number of assessors, experience of assessors (training, piloting, previous risk of bias assessment experience), as well as method(s) of assessment (independent or in duplicate) (30). Whether reviewers are going to be blinded to studies should also be reported, $(31,32)$ as well as whether agreement between reviewers will be evaluated and, if so, how.

Details of planned methods to summarize risk of bias assessments across studies or outcomes should be provided. Although authors may spend a large proportion of time assessing risk of bias in included studies, they are often silent on how the results might influence their review findings.

Thus, authors are encouraged to think about this at the development stage and document their plans in the protocol. Authors should also describe how risk of bias assessments will be incorporated into data synthesis (that is, subgroup or sensitivity analyses) and their potential influence on findings of the review in the protocol.

The likelihood that the treatment effect reported in a systematic review represents the true effect depends on the validity of the included studies, namely, the internal validity. Therefore, authors should not only describe risk of bias methods and constructs to be assessed for each included study, but also describe how results of the assessment contribute to the overall findings of the review (33). Additionally, authors should provide a rationale if they do not intend to assess risk of bias.

Many methods exist to assess the overall risk of bias in included studies, including scales, checklists, and individual components $(34,35)$. As summarized in the PRISMA elaboration document,17 scales that numerically summarize multiple components into a single number are misleading and unhelpful (36). Rather, authors should specify the methodological components that they plan to assess and how they plan to assess said components.

Authors should give careful consideration to assessments for reviews that expect to include nonparallel group randomised controlled trials and studies of non-randomised design, for which methodological standards are currently under development. The ultimate decision regarding which methodological features should be evaluated requires consideration of the strength of the empirical data, theoretical rationale, and the unique circumstances of the included studies within the context of the review question.

\subsubsection{Meta-bias(es):}

Authors should pre-specify any methods used to explore the possibility that the data identified are biased due to non-study related processes (37). Such bias may result from non-publication of studies (publication or dissemination bias) and the reporting of a subset of measured outcomes and analyses within studies (outcome reporting bias)

Systematic reviews aim to synthesize the results of all relevant studies. However, some studies may not be published, and a subset of outcomes and analyses may be incompletely, inadequately, or selectively reported in a published article, based on the results (such as statistical significance, 
magnitude, or direction of effect). The validity of systematic reviews may be threatened if the outcome data available to reviewers comprise a biased selection of all data that actually exists (38). Such biases are termed meta-biases, meaning that they occur independent of procedural problems during the conduct of a primary study as do typical methodological biases (such as inappropriate method of random sequence generation in randomized trials) (37).

In specific:

- Publication or dissemination bias - Several systematic reviews of empirical studies have found that clinical trials with statistically significant $(\mathrm{P}<0.05)$ or positive results are more likely to be published than those with non-significant or negative results (39-41). Investigators' decisions not to submit papers with negative results for publication, rather than editors' rejection of such papers, tend to be the main source of publication bias (42). However, the decision to write up a study for publication may be influenced by pressure from study sponsors and journal editor (25). Studies with statistically significant results also tend to be published earlier than studies with non-significant results (40). If studies are missing from a systematic review for these reasons, exaggerated results may be produced.

- Outcome reporting bias - The selective reporting of outcomes due to their significance, magnitude, or direction is termed outcome reporting bias and has been widely documented across the trial literature (39). Outcomes specified in the protocol may be completely omitted from the published report. When an outcome is measured using multiple scales or at multiple time points, and analysed in various ways (such as intention-to-treat and per-protocol analysis, unadjusted and adjusted for covariates), the choice of which data to present may be influenced by the results. Nonsignificant results may be partially reported (such as reporting an effect estimate with no measure of variation), resulting in insufficient data to include in a meta-analysis. All of these examples of selectively reported outcome data in primary studies can bias (and sometimes, overestimate) the results of systematic reviews $(39,26,43)$.

Empirical evidence of selective outcome reporting bias in trials exists. A systematic review of 16 cohorts of clinical trials comparing outcomes reported in trial protocols with the published reports found that at least one primary outcome was omitted, introduced, or changed in $4-50 \%$ of reports (44). In a landmark study, Chan and colleagues found that statistically significant outcomes had higher odds of being fully reported in trial publications compared with non-significant outcomes for efficacy (pooled odds ratio 2.4 (95\% confidence interval 1.4 to 4.0$)$ ) and safety (pooled odds ratio 4.7 (1.8 to 12$)$ ) (37).

Detecting or correcting for publication bias in a systematic review is difficult.

Recommendations regarding appropriate graphical methods (such as funnel plots) and statistical methods (such as Egger's test) to assess small study effects have been proposed (45).

However, publication bias is only one of several possible explanations for small study effects, and the interpretation of such tests can be problematic (45). Authors should report their planned testing strategy to assess publication bias in detail. 
For some trials, reviewers might assume that it is likely that an outcome was measured even if it was not reported, based on knowledge of the clinical area (such as when systolic, but not diastolic, blood pressure is reported) (46). Authors may use the Outcome Reporting Bias in Trials (ORBIT) classification system (47). A sensitivity analysis to assess the impact of selective reporting on metaanalytic results may also be considered (48).

For systematic reviews/meta-analysis the PRISMA-statement is recommended, as it gives the reader for a better understanding of the selection process (49)

In conclusion evidence based medicine is an important part of medicine and primary trials are the basis for higher level evidence trials and clinical decisions.

In future the use of trial registration for minimizing publication bias (50), mechanisms to allow easier access to the scientific literature and improvement in the peer review process are recommended. The use of checklists like PRISMA is likely to improve the reporting quality of a systematic review and provides substantial transparency in the selection process of papers in a systematic review.

\subsection{The PRISMA Statement:}

A systematic review is a review of a clearly formulated question that uses systematic and explicit methods to identify, select, and critically appraise relevant research, and to collect and analyze data from the studies that are included in the review. Statistical methods (meta-analysis) may or may not be used to analyze and summarize the results of the included studies. Meta-analysis refers to the use of statistical techniques in a systematic review to integrate the results of included studies.

The conduct of a systematic review depends heavily on the scope and quality of included studies, thus systematic reviewers may need to modify their original review protocol during its conduct. Any systematic review reporting guideline should recommend that such changes can be reported and explained without suggesting that they are inappropriate.

The PRISMA Statement acknowledges this iterative process. Aside from Cochrane reviews, all of which should have a protocol, only about $10 \%$ of systematic reviewers report working from a protocol. Without a protocol that is publicly accessible, it is difficult to judge between appropriate and inappropriate modifications.

PRISMA stands for Preferred Reporting Items for Systematic Reviews and Meta-Analyses (www.prisma-statement.org). It is an evidence-based minimum set of items for reporting in systematic which comes from a revision of the QUORUM checklist together with the addition of a flow diagram done during a three-day meeting held in Ottawa, Canada, in June 2005. Here, an international survey of review authors, consumers, and groups commissioning or using systematic reviews and meta-analyses was completed, including the International Network of Agencies for Health Technology Assessment (INAHTA) and the Guidelines International Network (GIN). The survey aimed to ascertain views of QUOROM, including the merits of the existing checklist items. The results of these activities were presented during the meeting and are summarized on the PRISMA Web site (http:// www.prisma-statement.org/) (49). 
Shortly after the meeting a draft of the PRISMA checklist was circulated to the group. A disposition file was created containing comments and revisions from each respondent, and the checklist was subsequently revised 11 times. The group approved the checklist, flow diagram, and a summary paper was written (49).

The PRISMA Statement consists of a 27-item checklist (Table 1) and a four-phase flow diagram (Figure 1) (51). 
Table 1. Preferred reporting items for systematic review and meta-analysis protocols 2015 checklist: recommended items to address in a systematic review protocol.

\begin{tabular}{|c|c|c|}
\hline Section and topic & Item No & Checklist item \\
\hline \multicolumn{3}{|l|}{ Administrative information } \\
\hline \multicolumn{3}{|l|}{ Title: } \\
\hline Identification & $1 \mathrm{a}$ & Identify the report as a protocol of a systematic review \\
\hline Update & $1 b$ & If the protocol is for an update of a previous systematic review, identify as such \\
\hline Registration & 2 & If registered, provide the name of the registry (such as PROSPERO) and registration number \\
\hline \multicolumn{3}{|l|}{ Authors: } \\
\hline Contact & 3a & $\begin{array}{l}\text { Provide name, institutional affiliation, e-mail address of all protocol authors; provide physical mailing address of } \\
\text { corresponding author }\end{array}$ \\
\hline Contributions & $3 b$ & Describe contributions of protocol authors and identify the guarantor of the review \\
\hline Amendments & 4 & $\begin{array}{l}\text { If the protocol represents an amendment of a previously completed or published protocol, identify as such and list } \\
\text { changes; otherwise, state plan for documenting important protocol amendments }\end{array}$ \\
\hline \multicolumn{3}{|l|}{ Support: } \\
\hline Sources & $5 a$ & Indicate sources of financial or other support for the review \\
\hline Sponsor & $5 b$ & Provide name for the review funder and/or sponsor \\
\hline Role of sponsor or funder & $5 c$ & Describe roles of funder(s), sponsor(s), and/or institution(s), if any, in developing the protocol \\
\hline \multicolumn{3}{|l|}{ Introduction } \\
\hline Rationale & 6 & Describe the rationale for the review in the context of what is already known \\
\hline Objectives & 7 & $\begin{array}{l}\text { Provide an explicit statement of the question(s) the review will address with reference to participants, interventions, } \\
\text { comparators, and outcomes (PICO) }\end{array}$ \\
\hline \multicolumn{3}{|l|}{ Methods } \\
\hline Eligibility criteria & 8 & $\begin{array}{l}\text { Specify the study characteristics (such as PICO, study design, setting, time frame) and report characteristics (such } \\
\text { as years considered, language, publication status) to be used as criteria for eligibility for the review }\end{array}$ \\
\hline Information sources & 9 & $\begin{array}{l}\text { Describe all intended information sources (such as electronic databases, contact with study authors, trial registers } \\
\text { or other grey literature sources) with planned dates of coverage }\end{array}$ \\
\hline Search strategy & 10 & $\begin{array}{l}\text { Present draft of search strategy to be used for at least one electronic database, including planned limits, such that } \\
\text { it could be repeated }\end{array}$ \\
\hline \multicolumn{3}{|l|}{ Study records: } \\
\hline Data management & $11 \mathrm{a}$ & Describe the mechanism(s) that will be used to manage records and data throughout the review \\
\hline Selection process & $11 b$ & $\begin{array}{l}\text { State the process that will be used for selecting studies (such as two independent reviewers) through each phase } \\
\text { of the review (that is, screening, eligibility and inclusion in meta-analysis) }\end{array}$ \\
\hline Data collection process & $11 \mathrm{c}$ & $\begin{array}{l}\text { Describe planned method of extracting data from reports (such as piloting forms, done independently, in duplicate), } \\
\text { any processes for obtaining and confirming data from investigators }\end{array}$ \\
\hline Data items & 12 & $\begin{array}{l}\text { List and define all variables for which data will be sought (such as PICO items, funding sources), any pre-planned } \\
\text { data assumptions and simplifications }\end{array}$ \\
\hline Outcomes and prioritization & 13 & $\begin{array}{l}\text { List and define all outcomes for which data will be sought, including prioritization of main and additional outcomes, } \\
\text { with rationale }\end{array}$ \\
\hline Risk of bias in individual studies & 14 & $\begin{array}{l}\text { Describe anticipated methods for assessing risk of bias of individual studies, including whether this will be done at } \\
\text { the outcome or study level, or both; state how this information will be used in data synthesis }\end{array}$ \\
\hline \multirow[t]{4}{*}{ Data synthesis } & $15 a$ & Describe criteria under which study data will be quantitatively synthesised \\
\hline & $15 b$ & $\begin{array}{l}\text { If data are appropriate for quantitative synthesis, describe planned summary measures, methods of handling data } \\
\text { and methods of combining data from studies, including any planned exploration of consistency (such as } I^{2} \text {, Kendall's } \\
\tau \text { ) }\end{array}$ \\
\hline & $15 \mathrm{c}$ & Describe any proposed additional analyses (such as sensitivity or subgroup analyses, meta-regression) \\
\hline & $15 d$ & If quantitative synthesis is not appropriate, describe the type of summary planned \\
\hline Meta-bias(es) & 16 & $\begin{array}{l}\text { Specify any planned assessment of meta-bias(es) (such as publication bias across studies, selective reporting within } \\
\text { studies) }\end{array}$ \\
\hline Confidence in cumulative evidence & 17 & Describe how the strength of the body of evidence will be assessed (such as GRADE) \\
\hline
\end{tabular}


Figure 1. Flow of information through the different phase of a systematic review.
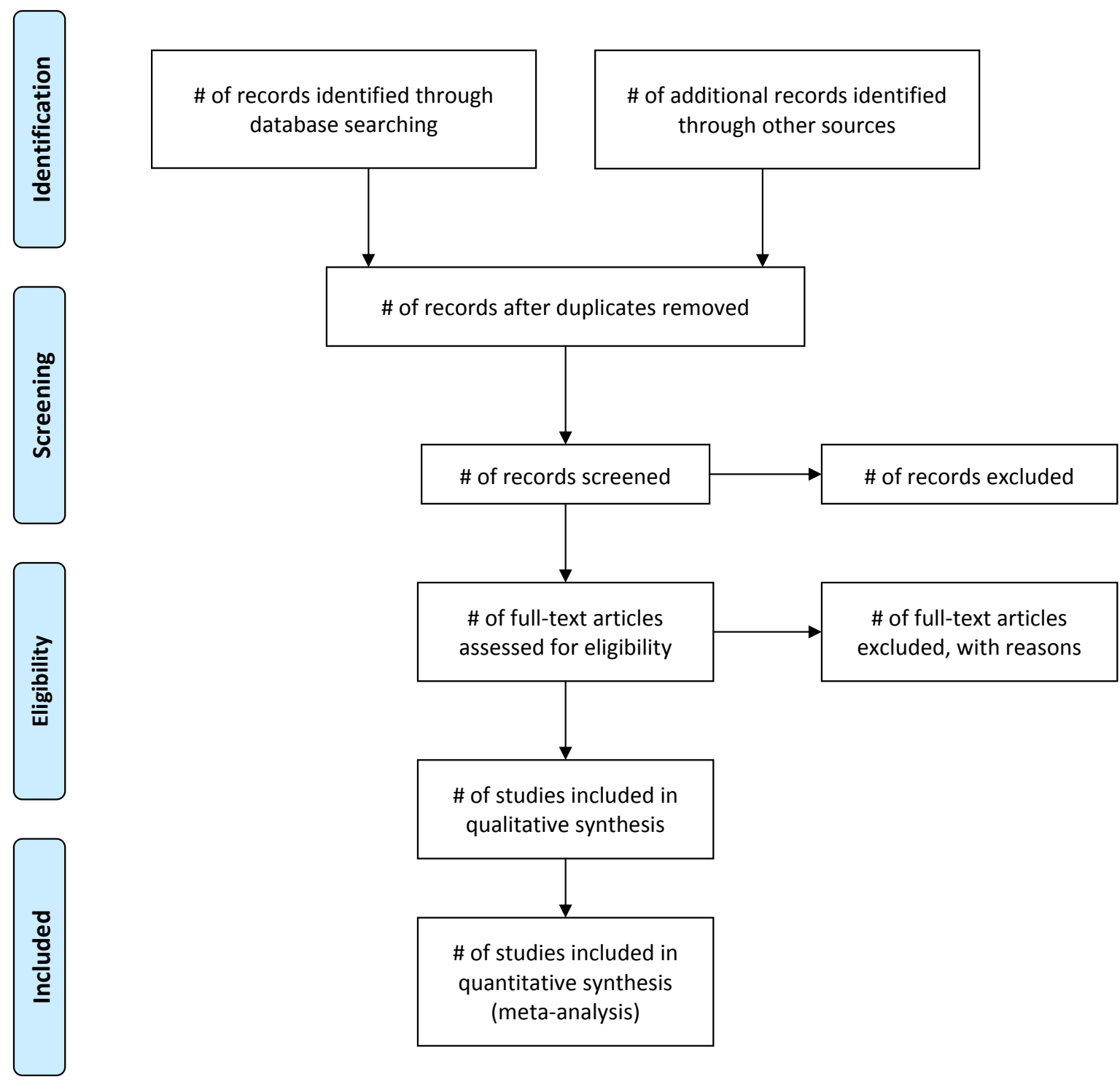
The new PRISMA checklist differs in several respects from the QUOROM checklist, and the substantive specific changes are highlighted in Table 2.

Table 2.

\begin{tabular}{|c|c|c|c|c|}
\hline Section/Topic & Item & QUOROM & PRISMA & Comment \\
\hline Abstract & & $\sqrt{ }$ & $\sqrt{ }$ & $\begin{array}{l}\text { QUOROM and PRISMA ask authors to report an abstract. However, } \\
\text { PRISMA is not specific about format. }\end{array}$ \\
\hline Introduction & Objective & & $\sqrt{ }$ & $\begin{array}{l}\text { This new item (4) addresses the explicit question the review } \\
\text { addresses using the PICO reporting system (which describes the participants, } \\
\text { interventions, comparisons, and outcome(s) of the systematic review), } \\
\text { together with the specification of the type of study design (PICOS); the item } \\
\text { is linked to Items } 6,11 \text {, and } 18 \text { of the checklist. }\end{array}$ \\
\hline Methods & Protocol & & $\sqrt{ }$ & $\begin{array}{l}\text { This new item (5) asks authors to report whether the review has } \\
\text { a protocol and if so how it can be accessed. }\end{array}$ \\
\hline Methods & Search & $\sqrt{ }$ & $\sqrt{ }$ & $\begin{array}{l}\text { Although reporting the search is present in both QUOROM and } \\
\text { PRISMA checklists, PRISMA asks authors to provide a full description } \\
\text { of at least one electronic search strategy (Item 8). Without such information } \\
\text { it is impossible to repeat the authors' search. }\end{array}$ \\
\hline Methods & $\begin{array}{l}\text { Assessment of risk of } \\
\text { bias in included studies }\end{array}$ & $\sqrt{ }$ & $\sqrt{ }$ & $\begin{array}{l}\text { Renamed from "quality assessment" in QUOROM. This item (12) } \\
\text { is linked with reporting this information in the results (Item 19). } \\
\text { The new concept of "outcome-level" assessment has been introduced. }\end{array}$ \\
\hline Methods & $\begin{array}{l}\text { Assessment of risk } \\
\text { of bias across studies }\end{array}$ & & $\sqrt{ }$ & $\begin{array}{l}\text { This new item (15) asks authors to describe any assessments of } \\
\text { risk of bias in the review, such as selective reporting within the included studies. } \\
\text { This item is linked with reporting this information in the results (Item 22). }\end{array}$ \\
\hline Discussion & & $\sqrt{ }$ & $\sqrt{ }$ & $\begin{array}{l}\text { Although both QUOROM and PRISMA checklists address the discussion } \\
\text { section, PRISMA devotes three items }(24-26) \text { to the discussion. In PRISMA } \\
\text { the main types of limitations are explicitly stated and their discussion required. }\end{array}$ \\
\hline Funding & & & $\sqrt{ }$ & $\begin{array}{l}\text { This new item (27) asks authors to provide information on any sources of funding } \\
\text { for the systematic review. }\end{array}$ \\
\hline
\end{tabular}

Generally, the PRISMA checklist "decouples" several items present in the QUOROM checklist and, where applicable, several checklist items are linked to improve consistency across the systematic review report.

The flow diagram has also been modified. Before including studies and providing reasons for excluding others, the review team must first search the literature. This search results in records. Once these records have been screened and eligibility criteria applied, a smaller number of articles will remain. The number of included articles might be smaller (or larger) than the number of studies, because articles may report on multiple studies and results from a particular study may be published in several articles. To capture this information, the PRISMA flow diagram now requests information on these phases of the review process.

The aim of the PRISMA Statement is to help authors improve the reporting of systematic reviews and meta-analyses. PRISMA can also be used as a basis for reporting systematic reviews of other types of research, particularly evaluations of interventions and it may also be useful for critical appraisal of published systematic reviews. However, the PRISMA checklist is not a quality assessment instrument to gauge the quality of a systematic review.

\section{Laryngeal lesions:}

\subsection{Epithelial precursor lesions}

\subsubsection{Definition}

Precursor lesions are defined as altered epithelium with an increased likelihood for progression to squamous cell carcinoma (SCC). The altered epithelium shows a variety of cytological and 
architectural changes that have traditionally been grouped under the term dysplasia. Rarely, malignant transformation can develop even from morphologically normal epithelium. Atypia is not considered synonymous with dysplasia (Table 3).

Table 3. Classification schema that histologically categorizes precursors and related lesions.

\begin{tabular}{|c|c|c|}
\hline 2005 WHOClassification & $\begin{array}{l}\text { Squamous Intraepithelial } \\
\text { Neoplasia (SIN) }\end{array}$ & $\begin{array}{l}\text { Ljubljana Classification Squamous } \\
\text { Intraepithelial Lesions (SIL) }\end{array}$ \\
\hline Squamous cell hyperplasi & & Squamous cell (simple) hyperplasia \\
\hline Mild dysplasia & SIN 1 & Basal/Parabasal cell hyperplasia* \\
\hline Moderate dyplasia & SIN 2 & Atypical hyperplasia** \\
\hline Severe dysplasia & $\operatorname{SIN} 3 * * *$ & Atypical hyperplasia** \\
\hline Carcinoma in-situ & $\operatorname{SIN} 3 * * *$ & Carcinoma in-situ \\
\hline \multicolumn{3}{|c|}{$\begin{array}{l}\text { * Basal/parabasal cell hyperplasia may histologically resemble mild dysplasia, but the former is } \\
\text { conceptually benign lesion and the latter the lower grade of precursor lesions; } \\
\text { ** "Risky epithelim"; the analogy to moderate and severe dysplasia is approximate; } \\
\text { ** The advocates of SIN combine severe dysplasia and carcinoma in-situ. }\end{array}$} \\
\hline
\end{tabular}

Atypia has been used in the context of inflammatory and regenerative changes particularly referring to cytologic features. In this text, the term atypia refers to cytological change that may or may not be pre-malignant. Various classifications have evolved to describe the spectrum of histological changes in relation to their malignant potential (52).

\subsubsection{Epidemiology}

The entire spectrum of laryngeal and hypopharyngeal precursor lesions are mostly seen in the adult population and affect men more often than women. This gender disparity is especially pronounced after the sixth decade (53). Mean ages for the first precursor lesion diagnosis are reported from 48.0-56.5 years $(53,54)$. The incidence varies worldwide with the magnitude and manner of carcinogen exposure.

\subsubsection{Etiology}

Precursor lesions are strongly associated with tobacco smoking and alcohol abuse, and especially a combination of these two (55-58). The risk of developing these lesions increases with duration of smoking, the type of tobacco and the practice of deep inhalation.

Additional etiological factors are: industrial pollution, specific occupational exposures, nutritional deficiency and hormonal disturbance $(54,55,59-61)$. The role of human papilloma virus (HPV) infection is still unsolved (62).

\subsubsection{Localization}

Precursor lesions appear mainly along the true vocal cords. Two thirds of vocal cord lesions are bilateral (53). They can extend over the free edge of the vocal cord to the subglottic surface. An 
origin in, or extension along the upper surface. of the vocal cord is less common $(54,63)$. The commissures are rarely involved (53).

\subsubsection{Clinical features}

Most patients with precursor lesions give a history of a few months or more of symptoms, but may be asymptomatic (53). Symptoms depend on the location and severity of the disease and include fluctuating hoarseness, throat irritation, sore throat, and/or chronic cough. Precursor lesions can be either sharply circumscribed and grow exophytically, or be predominantly flat and diffuse, related in part to the amount of keratin present.

\subsubsection{Macroscopy}

Precursor lesions have a clinically diverse appearance, variously described as leukoplakia (white patch), chronic hyperplastic laryngitis or rarely erythroplasia/erythroplakia (red patch). A circumscribed thickening of the mucosa covered by whitish patches, or an irregularly growing, well-defined warty plaque may be seen. A speckled appearance of lesions can also be present, caused by unequal thickness of the keratin layer. However, the lesions are commonly more diffuse, with a thickened appearance, occupying a large part of one or both vocal cords. Their surface is rough, may be muddy brown to red (erythroplasia), perhaps with increased visible vascularity, or coated with diffuse or dispersed circumscribed whitish plaques (speckled leukoplakia) (54). Few white patches are ulcerated (6.5\%) or combined with erythroplasia (15\%) (53). Leukoplakia, in contrast to erythroplasia, tends to be well demarcated. In general, leukoplakia has a lower risk of malignant transformation than mixed white and red lesions, or speckled leukoplakia, which has an intermediate risk, and pure erythroplasia which has the highest risk of cancer development (64). However, no one clinical appearance is reliably diagnostic of any histologic grade of precursor lesion. Occasionally precursor lesions may appear clinically normal.

\subsubsection{Histopathology}

The epithelium of all precursor lesions is generally thickened. However, in a minority of cases patchy atrophy, thinning of the viable cellular layers, may be present. By definition there is no evidence of invasion. The magnitude of surface keratinization is of no importance. Allocation to categories within each of the classifications requires consideration firstly of architectural features and then of cytology.

\section{- Hyperplasia:}

describes increased cell numbers. This may be in the spinous layer (acanthosis) and/or in the basal/ parabasal cell layers (progenitor compartment), termed basal cell hyperplasia. The architecture shows regular stratification and there is no cellular atypic (Figure 2). 
Figure 2. Squamous cell hyperplasia (simple hyperplasia). There is an increased number of ordinary- arranged, otherwise normal cells in the spinous layer. A keratin layer is present on the surface.

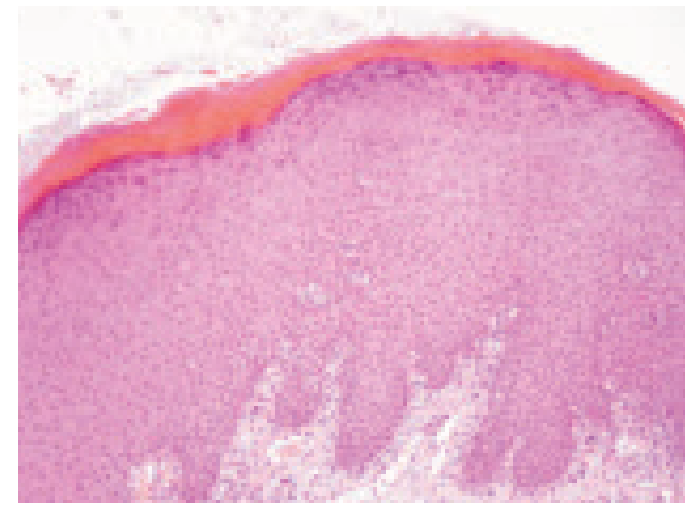

\section{- Dysplasia (intraepithelial neoplasia, atypical epithelial hyperplasia potentially malignant lesions):}

When architectural disturbance is accompanied by cytologic atypia the term dysplasia applies (Table 4).

Table 4. Criteria used for diagnosing dysplasia.

\begin{tabular}{|l|l|}
\hline Architecture & Cytology \\
\hline Irregular epithelial stratification & Abnormal variation in nuclear size (anisonucleosis) \\
\hline Loss of polarity of basal cells & $\begin{array}{l}\text { Abnormal variation in nuclear shape (nuclear } \\
\text { pleomorphism) }\end{array}$ \\
\hline Drop-shape rete ridges & Abnormal variation in cell size (anisocytosis) \\
\hline Increased number of mitotic figures & Abnormal variation in cell shape (cellular pleomorphism) \\
\hline Abnormal superficial mitoses & Increased nuclear-cytoplasmic ratio \\
\hline Premature keratinization in single cells (dyskeratosis) & Increased nuclear size \\
\hline Keratin pearls within rete pegs & Atypical mitotic figures \\
\hline & Increased number and size of nucleoli \\
\hline
\end{tabular}

There is a challenge in the recognition of the earliest manifestations of dysplasia, and no single combination of the above features allows for consistent distinction between hyperplasia and the earliest stages of dysplasia as well as in attempting to rigidly divide the spectrum of dysplasia into mild, moderate and severe categories: 


\section{Mild dysplasia}

In general architectural disturbance limit- ed to the lower third of the epithelium accompanied by cytological atypia define the minimum criteria of dysplasia (Figure 3a,b).

\section{Moderate dysplasia}

Architectural disturbance extending into the middle third of the epithelium is the initial criterion for recognizing this category. However, consideration of the degree of cytologic atypia may require upgrading (Figure 3c,d)

Severe dysplasia

Recognition of severe dysplasia starts with greater than two thirds of the epithelium showing architectural disturbance with associated cytologic atypia. However, architectural disturbance extend- ing into the middle third of the epithelium with sufficient cytologic atypia may be upgraded from moderate to severe dysplasia (Figure 3e).

\section{Carcinoma in-situ}

The theoretical concept of carcinoma in- situ is that malignant transformation has occurred but invasion is not present. It is not always possible to recognize this morphologically. The following is recommended for the diagnosis of carcinoma in-situ: full thickness or almost full thick- ness architectural abnormalities in the viable cellular layers accompanied by pronounced cytologic atypia. Atypical mitotic figures and abnormal superficial mitoses are commonly seen in carcinoma in-situ (Figure 3f).

Figure 3. Types of dysplasia: a) Mild dysplasia (SIN1): note the increased number of basalparabasal cells with hyperchromatic, uniform nuclei, perpendicularly oriented to the basement membrane. The upper part of the epithelium shows a regular spinous layer and thin parakeratotic layer on the surface; b) Mild dysplasia (SIN1): Increased number of uniform, slightly enlarged basal and parabasal cells, perpendicularly oriented to the basement membrane. Increased number of regular mitoses are evident. At the right corner (lower half) the epithelial cells show minimal cytologic atypia. The upper half of the epithelium is composed of regular spinous cells, which become flattened toward the surface. A thin parakeratotic layer is present on the surface; c) Moderate dysplasia (SIN2): the epithelium is slightly thickened. More than half of the epithelium is occupied by increased number of slightly to moderately polymorphic epithelial cells with hyperchromatic nuclei and increased mitotic activity. The upper third shows spinous cell differentiation; prominent granular and keratotic layer is evident on the surface; d) Moderate dysplasia (SIN2): Hyperplastic epithelium with increased number of slightly to moderately polymorphic epithelial cells extending up to two thirds of the epithelium. A thin parakeratotic and keratotic layer is present on the surface; e) Severe dysplasia (SIN 3, atypical hyperplasia). The atypical epithelial cells occupy two thirds of the epithelial thickness. Note partially preserved epithelial stratification, expressed cytologic atypia and increased mitotic activity. Keratin layer is present on the surface; f) Carcinoma in-situ (SIN 3): prominent architectural disarray, marked cytologic atypia and increased mitotic figures with pathologic forms. 

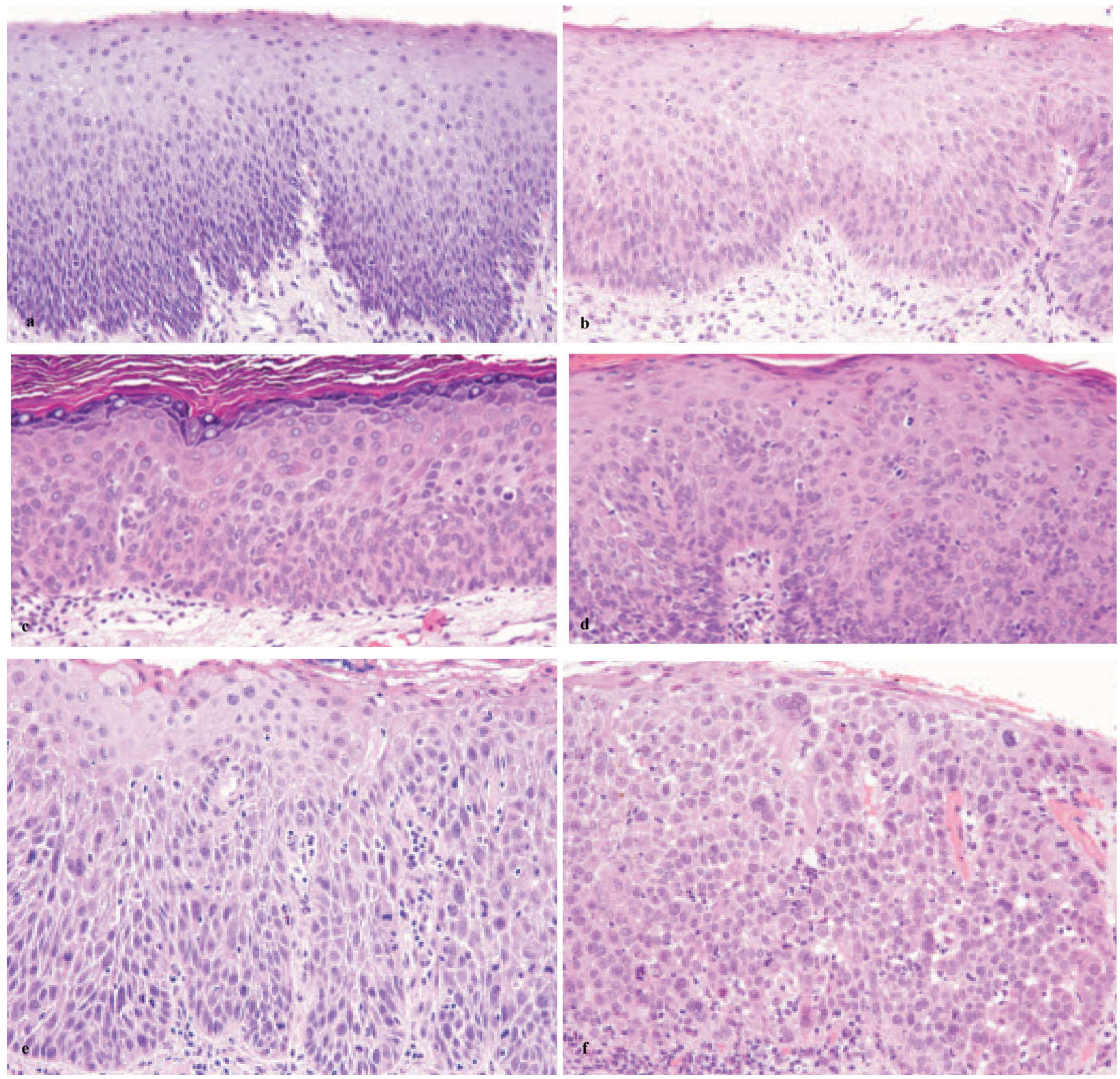

\subsubsection{Differential diagnosis}

Reactive, regenerative or reparative squamous epithelium (for example in response to trauma, inflammation, irradiation or ulceration) may manifest atypical cytology or architectural disturbance. Nutritional deficiencies such as iron, folate, and vitamin B12, can also simulate dysplasia. Such lesions are not considered precursor lesions and should be distinguished from them. Clinical history is helpful, and morphologic changes suggestive of the inciting event (e.g. ulceration, inflammation, haemorrhage, radiation-induced mesenchymal and/or endothelial nuclear enlargement and hyperchromasia) may be present. The epithelial changes in these cases are generally less pronounced than in severe dysplasia/atypical hyperplasia or CIS, atypical mitoses are almost never present, and the epithelium may be thinned, or, if thickened, stratification and maturation often develop as the regenerative/ reparative process matures. 


\subsubsection{Prognosis and predictive factors}

Some precursor lesions are self-limiting and reversible, others persist and some progress to SCC (65). The histopathologic degree of severity of these lesions can be a predictive factor (66). Simple and basal/parabasal cell hyperplasias have a minimal likelihood of malignant progression (0.9\%). These patients do not require close clini- cal follow-up. Lesions classified as atypical hyperplasia (moderate to severe dysplasia) have a $11 \%$ rate of malignant transformation (66). Diagnosis of precursor lesions implies a need for close follow-up and complete excision depending on the clinical situation (66). Patients with carcinoma in-situ require more extensive management, depending on the clinical circumstance $(54,65)$.

\subsection{Laryngeal cancer}

\subsubsection{Epidemiology}

Laryngeal and hypopharyngeal squamous cell carcinoma (SCC) occur most frequently in the sixth and seventh decades, but some cases have been described in children $(67,68)$. They are more common in men $(69,70)$ though the male:female ratio is decreasing in some countries; women are becoming increasingly affected because of increased prevalence of smoking over the last two decades (71).

\subsubsection{Incidence}

SCC comprises about $95 \%$ of laryngeal malignancies. The majority originate from the supraglottic and glottic regions, although there are geographic variations in the relative ratio between these two sites. The incidence in men is high $(10 / 100,000$ pa or more) in southern and central Europe, southern Brazil, Uruguay and Argentina and among Blacks in the United States. The lowest rates $(<1 / 100,000 \mathrm{pa})$ are recorded in South- East Asia and central Africa. The incidence in women is below 1/100,000 pa in most populations. An estimated 140,000 new cases occurred worldwide in $1990,86 \%$ of these patients were men $(72,73)$. The incidence is slightly more common in urban that in rural areas $(69,70)$.

There are also geographic differences in the topographic distribution of the laryngeal SCC. In France, Spain, Italy, Finland and the Netherlands, supraglottic SCC predominates, while in the United States, Canada, England and Sweden glottic SCC is more common. In Japan, SCC is approximately equally distributed between the two sites. Interpretation of incidence rates of hypopharyngeal cancer is probably complicated by absence or misclassifications within sub sites of the larynx. Recorded incidence is highest among men $(>2.5 / 100,000 \mathrm{pa})$ in India, Brazil and Central and Western Europe, and is lowest $(<0.5 / 100,000 \mathrm{pa})$ in East Asia, Africa and Northern Europe. Incidence among women is low $(<0.2 / 100,000 \mathrm{pa})$ in most populations except India, where rates up to $1 / 100,000$ pa are recorded (74). This is probably due to the fact that tobacco is more often chewed than smoked in India. The incidence of laryngeal and hypopharyngeal SCC is increasing in much of the world, both in men and in women. This increase is related to changes in tobacco and alcohol consumption (69).Primary prevention of laryngeal and hypopharyngeal SCC could be achieved by cessation of smoking and reduction of alcohol consumption (69). 


\subsubsection{Etiology}

\section{- Tobacco and alcohol:}

Most cases of laryngeal cancer in Western countries are related to smoking and alcohol abuse. The combined effect follows a multiplicative rather than additive model (75). The increased relative risk (RR) for alcohol consumption differs by site, and is higher for the supraglottis and hypopharynx and lower for the glottis and subglottis (76). The impact of increased RR (10x) for smoking is stronger for glottic than supraglottic SCC (76). Studies in several populations have shown a direct doserelated response between smoking and SCC and the benefits of cessation. Smoking black tobacco cigarettes entails a stronger risk than smoking blond tobacco (77). Other smoking habits that increase the RR of laryngeal SCC include: smoking at a young age, long duration, high number of cigarettes per day, and deep smoke inhalation (78). The influence of tobacco on RR of laryngeal SCC is confirmed even for non-drinkers. Case controlled studies from Italy and Switzerland show an increased RR of 2.46 for heavy drinkers and laryngeal SCC. The RR for current smokers who do not drink is 9.38 (79). Avoiding cigarettes and alcohol could prevent about $90 \%$ of laryngeal and hypopharyngeal SCC.

- Asbestos and occupational exposure:

There is controversy regarding occupational asbestos exposure and increased risk for developing laryngeal SCC (80). However, there is evidence supporting other occupational exposures and increased risk of laryngeal SCC, such as polycyclic aromatic hydrocarbons, metal dust, cement dust, varnish, lacquer, etc. After adjustment for alcohol and tobacco consumption, the increased risk ranged from 1.8 for cement dust to 2.7 for polycyclic aromatic hydrocarbons. Significant associations are also found with ionizing radiation, diesel exhausts, sulphuric acid mists and mustard gas (60).

- Diet and nutritional factors:

A protective effect is probably exerted by high intake of fruits and vegetables (81). Specific evidence regarding carotenoids and vitamin $\mathrm{C}$, is inadequate for a conclusion (82).

- Gastropharyngeal reflux:

Gastroesophageal reflux has been related to increased risk of laryngeal SCC, especially among patients who lack other major risk factors (83). Gastroesophageal reflux may act as a promoter in the presence of tobacco and alcohol (84).

- Genetic susceptibility:

There is no evidence of strong genetic factors in laryngeal carcinogenesis; however, polymorphisms for enzymes implicated in the detoxification of alcohol and tobacco, such as alcohol and aldehyde dehydrogenases, are likely to represent weak susceptibility factors, with relative risks in the order of 1.5-2 (85). Bloom syndrome is an inheritable condition with a predisposition towards laryngeal and hypopharyngeal SCC.

\section{- Prognosis and predictive factors:}

Small glottic or supraglottic SCC can be treated conservatively by laser excision, limited resection, or primary radiotherapy (RT), with curative potential, and overall good survival. RT failures can be salvaged by conservative, potentially curative voice-sparing surgery. Glottic or supraglottic 
carcinomas that fix the vocal cord(s) can be treated either by primary resection, with possible adjuvant RT, or organ sparing protocols (neoadjuvant chemotherapy with curative RT). If the carcinoma persists or recurs, overall survival is not compromised by delayed, salvage, total laryngectomy.

The TNM tumor classification (Table 5) consistently correlates, on multivariate survival analyses, with disease-free and overall survival. Among TNM stage IV patients, extensive cartilage invasion and/or bulky tumor volume are predictors of poor response to chemoradiotherapy; these patients are best treated with primary resection and possible adjuvant RT. Clinical comorbidities have been demonstrated to significantly affect survival over TNM prognosticators. The Washington University Head and Neck Comorbidity Index incorporates seven conditions (congestive heart disease, cardiac arrhythmia, peripheral vascular disease, pulmonary disease, renal disease, cancer controlled, and cancer uncontrolled) weighted according to severity, and is a significant predictor of survival.

Generally, histological classification (Table 6) and grading has limited impact on survival. By contrast, the pattern of tumor invasion at the advancing host/tumour interface has been demonstrated, by itself or in combination with other histological variables, to have predictive value for laryngeal carcinoma. Thus within T1/T2 laryngeal SCC, biopsy assessment of the pattern of invasion, may be utilized to predict which patients may respond to primary RT, versus which patients are better treated by primary resection. In multivariate analysis, overexpression of p53 is predictive of improved overall survival. p53 overexpression and elevated PCNA (proliferating cell nuclear antigen) index have been demonstrated to be significant independent predictors of successful organ preservation. 
Table 5. TNM classification of carcinomas of the larynx.

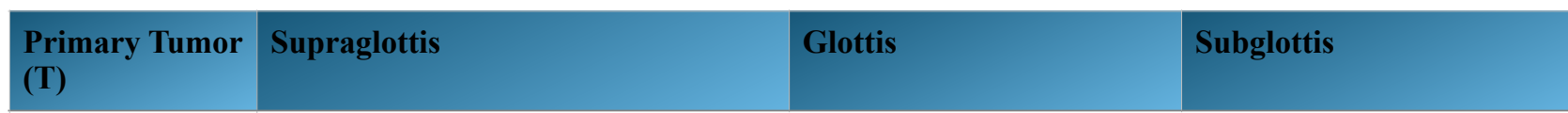

Tx

Primary tumor cannot be assessed.

T0

There is no evidence of primary tumor

Tis

Carcinoma is in situ.

$\mathrm{T} 1$

Tumor id limited to one sub site of the supra glottis, with normal vocal cord mobility.

Tumor is limited to the vocal cords(s) (may involve anterior or posterior commissure), with normal mobility: T1a is limited to one vocal cord; $\mathrm{T} 1 \mathrm{~b}$ involves both vocal cords.

Tumor invades mucosa of more than one adjacent subsite of the supraglottis or glottis or region outside the supraglottis (e.g., mucosa of base of tongue, vallecula, medial wall of pyriform sinus), without fixation of the larynx.

T3 Tumor is limited to the larynx with vocal cord fixation and/or invades any of the following: postcricoid area, preepiglottic tissues, paraglottic space, and/or minor thyroid cartilage erosion (e.g., inner cortex)
Tumor extends to the supraglottis and/or subglottis, and/or with mobility.

Tumor is limited to the larynx with vocal cord fixation and/or invades paraglottic space, and or minor thyroid cartilage erosion (e.g., inner cortex). impaired vocal cord
Tumor is limited to the

subglottis.

Tumor extends to the vocal cord(s), with normal or impaired mobility.

Tumor is limited to the larynx, with vocal cord fixation.

Tumor invades through the thyroid cartilage and/or invades tissues beyond the larynx (e.g., trachea, soft tissues of neck, including deep extrinsic muscle of the tongue, strap muscles, thyroid, or esophagus).

T4b Tumor invades pre vertebral space, encases the carotid artery, or invades mediastinal structures.

\begin{tabular}{l|l|l}
$\begin{array}{l}\text { Neck Staging } \\
\text { (N) }\end{array}$ & Supraglottis & Glottis \\
\hline Nx & Regional lymph nodes cannot be assessed \\
\hline N0 & There is no regional nodes metastasis
\end{tabular}

N1 Metastasis in a single ipsilateral lymph node, $3 \mathrm{~cm}$ of less in greatest dimension

N2 Metastasis in a single ipsilateral lymph node, more than $3 \mathrm{~cm}$ but not more than $6 \mathrm{~cm}$ in greatest dimension; or metastasis is in multiple ipsilateral lymph nodes, none more that $6 \mathrm{~cm}$ in greatest dimension; or metastasis is in bilateral or contralateral lymph nodes, none greater than $6 \mathrm{~cm}$ in greatest dimension.

N2a $\quad$ Metastasis in a single ipsilateral lymph node, more than $3 \mathrm{~cm}$ but not more than $6 \mathrm{~cm}$ in greatest dimension.

$\mathrm{N} 2 \mathrm{~b} \quad$ Metastasis in multiple ipsilateral lymph nodes, none more that $6 \mathrm{~cm}$ in greatest dimension

$\mathrm{N} 2 \mathrm{c}$

Metastasis in bilateral or contralateral lymph nodes, none more than $6 \mathrm{~cm}$ in greatest dimension

Metastasis in a lymph node more than $6 \mathrm{~cm}$ in greatest dimension 
Table 6. WHO histological classification of tumors of the larynx.

\begin{tabular}{|c|c|c|c|}
\hline Malignant epithelial tumours & & Rhabdomyosarcoma & $8900 / 3$ \\
\hline Squamous cell carcinoma & $8070 / 3$ & Angiosarcoma & $9120 / 3$ \\
\hline Verrucous carcinoma & $8051 / 3$ & Kaposi sarcoma & $9140 / 3$ \\
\hline Basaloid squamous cell carcinoma & $8083 / 3$ & Malignant peripheral nerve sheath tumour & \\
\hline Papillary squamous cell carcinoma & $8052 / 3$ & Synovial sarcoma & $9040 / 3$ \\
\hline Spindle cell carcinoma & $8074 / 3$ & Borderline tumours / LMP & \\
\hline Acantholytic squamous cell carcinoma & $8075 / 3$ & Inflammatory myofibroblastic tumour & $8825 / 1$ \\
\hline Adenosquamous carcinoma & $8560 / 3$ & Benign tumours & \\
\hline Lymphoepithelial carcinoma & $8082 / 3$ & Schwannoma & $9560 / 0$ \\
\hline Giant cell carcinoma & $8031 / 3$ & Neurofibroma & $9540 / 0$ \\
\hline Malignant salivary gland-type tumours & & Lipoma & $8850 / 0$ \\
\hline Mucoepidermoid carcinoma & $8430 / 3$ & Leiomyoma & $8890 / 0$ \\
\hline \multirow{2}{*}{ Adenoid cystic carcinoma } & $8200 / 3$ & Rhabdomyoma & $8900 / 0$ \\
\hline & & Hemangioma & $9120 / 0$ \\
\hline Neuroendocrine tumours & & Lymphangioma & $9170 / 0$ \\
\hline Typical carcinoid & $8240 / 3$ & Granular cell tumour & $9580 / 0$ \\
\hline Atypical carcinoid & $8249 / 3$ & & \\
\hline Small cell carcinoma, neuroendocrine type & $8041 / 3$ & Haematolymphoid tumours & \\
\hline \multirow[t]{2}{*}{ Combined small cell carcinoma, neuroendocrine type } & $8045 / 3$ & & \\
\hline & & Tumours of bone and cartilage & \\
\hline Benign epithelial tumours & & Chondrosarcoma & $9220 / 3$ \\
\hline Papilloma & $8050 / 0$ & Osteosarcoma & $9180 / 3$ \\
\hline Papillomatosis & $8060 / 0$ & Chondroma & $9220 / 0$ \\
\hline Salivary gland-type adenomas & & Giant cell tumour & $9250 / 1$ \\
\hline Pleomorphic adenoma & $8940 / 0$ & & \\
\hline Oncocytic papillary cystadenoma & $8290 / 0$ & Mucosal malignant melanoma & $8720 / 3$ \\
\hline Soft tissue tumours & & Secondary tumours & \\
\hline \multicolumn{4}{|l|}{ Malignant tumours } \\
\hline Fibrosarcoma & $8810 / 3$ & & \\
\hline Malignant fibrous histiocytoma & $8830 / 3$ & & \\
\hline Liposarcoma & $8850 / 3$ & & \\
\hline Leiomyosarcoma & $8890 / 3$ & & \\
\hline
\end{tabular}

\section{Laryngeal diagnostic systems and techniques.}

In patients with dysphonia, the diagnostic accuracy from history and physical exam, excluding laryngoscopy, is only 5\% compared with a $68.3 \%$ accuracy following an initial endoscopic laryngeal evaluation (86). Because of the wide range of potential causes for the dysphonia, determining the precise etiology of the laryngeal/voice disorder is necessary to plan treatment. Since malignant transformation rate of premalignant lesions ranges from $6 \%$ to $22 \%$, and it increases with the severity of the precancerous nature, their early detection is of paramount importance.

For instance, survival rates are significantly higher for early stage carcinomas, therefore, it is essential to concentrate on the initial steps in tumor development in order to facilitate early detection and timely implementation of suitable therapy (87). Early detection and preoperative assessment are important to a curative and function-preserving therapy, because the treatment of laryngeal cancer and its precursor lesions has a great impact on important basic functions of daily life such as breathing, verbal communication and swallowing. Furthermore, delayed diagnosis, leading to loco-regional failure, and a high incidence of second primary are the two main reasons for poor outcome.

From these observations, it is obvious that obtaining images of high quality and resolution, revealing the detailed morphology of the glottal structures, is one of the main tasks in laryngeal 
imaging. Such images are essential in making correct diagnosis and choosing the treatment to gain the best result.

Nowadays, there is a wide choose of different diagnostic tools, but each of them presents specific properties and limits that influence their usage in common practice. Herein a summary of their characteristic.

\subsection{Endoscopy}

Over time, laryngeal photography was perfected, which enhanced diagnostic accuracy, documentation, and education. Shortly thereafter, in 1895, both stroboscopy and direct laryngoscopy were introduced, which dramatically advanced laryngoscopy and laryngology (88). In the early 20th century, direct laryngoscopy and endolaryngeal surgery migrated to the operating room for the promise of improved surgical precision (89).

In laryngological and phoniatric diagnostics, white light laryngoscopy (WL) is the key procedure for functional investigation and early detection of neoplastic tissue (90). In fact, at present, white light laryngoscopy combined with biopsy is the standard diagnostic procedure in the assessment of laryngeal cancer and precancerous lesions. However, white light laryngoscopy provides poor quality images and has difficulty identifying minute epithelial changes and directly differentiating benign from malignant tumors in vivo.

Furthermore, with imprecise laryngeal diagnostics and lack of clinical experience, very early stages of malignant lesions, such as dysplasia or carcinoma in situ (CIS), can be overlooked, because they often present themselves as low-contrast mucosal changes with superficial roughness or reddening and rarely have an obviously characteristic malignant aspect (91).

\subsection{Stroboscopy}

Stroboscopy is considered to be an important part of diagnosing patients with laryngeal dysplasia. Nevertheless, we must note that a strict correlation between a vocal fold vibratory pattern and a certain type of lesion does not exist. Vocal fold pathology may produce changes in the appearance and vibratory patterns observed during stroboscopic examination. Interpreting the stroboscopic examination involves systematic judgment and describing the different vibratory pattern signs. These signs, which were first identified by Hirano and Bless (92) included the fundamental frequency and periodicity, amplitude of horizontal excursion, glottal closure, symmetry of bilateral movement, mucosal wave, and non vibrating portions of the vocal fold.

Quantitative characterization of the vibratory behavior of the vocal folds is a pivot task to add to the diagnostic step. A well-known drawback of video-laryngo-stroboscopy (VLS) is the reliance on quasi-periodic voice signals (93) when producing a real-time slow-motion stroboscopic effect. Objective measurements of the vocal fold vibration pattern date back to the initial high-speed cinematography recordings of vocal fold vibration from the works of Timcke, von Leden, and Moore (94-97).

Nowadays, because of the multiple factors related to the complex vocal fold vibratory system the actual clinical application of objective measurements has not yet been achieved. 
Several indices, describing the glottal wave form, are usually used for the characterization. They provide mainly the "functional information" about the vibratory function of the vocal folds and the glottal closure, and when they are matched with direct endoscopic images, they provide together more detailed information about structural and morphological peculiarities which are both fundamental in making the correct diagnosis. In fact, this tool is able to reveal a number of abnormalities, including abnormalities of laryngeal structure, absence of vibration, and vibratory asymmetry. Flashing light is used to illuminate an object in stroboscopy. When the flashes are synchronized with the vocal fold vibrations, a stationary view of the vocal folds is obtained. However, the single-flash-timing laryngeal videostroboscopy has a limitation that it is effective only when vocal fold vibrations exhibit only one single fundamental frequency. Multiple tones (fundamental frequencies) may be recorded in the presence of some diseases, such as polyps, nodules, cysts or precancerous lesions (98).

Quantitative measures of motion and geometry of vocal folds can provide objective information and may be useful in planning medical treatment and tracing progress over time. Few studies have indicated which stroboscopic signs are more significant than others in evaluating the vibratory pattern of vocal folds with premalignant lesions. Despite there some known features that cannot be imaged with stroboscope, such as voice breaks, diplophonia, vocal function during voice onset and voice offset, vocal tremor and spasms, extremely rough voice quality, alternate laryngeal and pharyngeal sources of oscillation (99), the main limitation with the VLS remains the subjective nature of the interpretation of laryngeal phonatory function examination results, which significantly reduces the reproducibility and the use of VLS as a research tool or even as a quantitative instrument for comparing outcomes of treatment of voice and laryngeal disorders. In fact, there is a lack of data in the literature about the specificity and sensitivity of VLS parameters discriminating normal and pathological voices.

The discrepancy in diagnosis with VLS seems to be linked to certain key points: (1) during office endoscopy, tangential views of the medial surface of the glottis limit the diagnostic sensitivity; (2) sulci and mucosal bridges are most subject to this limitation; (3) informed consent should address the potential need for a change in intraoperative management. It is advisable to discuss the possibility for dissection in both vocal folds, even if a unilateral lesion is observed in the office; (4) suspension microlaryngoscopy (SML) is the final diagnostic step in the evaluation of glottic pathology (100).

\subsection{Contact endoscopy}

Contact endoscopy (CE), first described in 1979 by Hamou (101), offers an additional in vivo diagnostic procedure based on the staining of the superficial mucosal layer and direct in vivo and in situ examination of the epithelial cells. The basic technique of $\mathrm{CE}$ involves staining of the superficial cells of the mucosa with $1 \%$ of nontoxic methylene blue before the magnification of the suspected areas through the direct contact of the tip of an endoscope to the mucosal surface to obtain cytological images (102). CE enables visualization of the laryngeal mucosa pathology through high magnification and therefore detailed examination of cells and blood vessels without 
requiring tissue biopsy. This non invasive method allows in vivo visualization of vascular alterations of the mucosa and rates either as benign or malignant.

The microvascular architectural changes seen with CE are generally classified in five different vascular patterns described in Table 7.

Table 7. Enhanced contact endoscopy vascular patterns.

\begin{tabular}{|c|c|c|}
\hline Vascular Pattern & Diagnosis & Description \\
\hline Type 0 & Normal mucosa & $\begin{array}{l}\text { Thin-end regular subepithelial vessels connecting with a thicker and deeper } \\
\text { arborescent vascular network running parallel to the epithelium. }\end{array}$ \\
\hline Type I & Inflammation & $\begin{array}{l}\text { The subepithelial vessels are increased in number and size, with irregular and } \\
\text { sometimes crossing directions. }\end{array}$ \\
\hline Type II & Hyperplasia & $\begin{array}{l}\text { When the hyperplasia is at the initial stage, intra-CLs are visible running toward } \\
\text { the surface. In this phase, CLs are generally still very thin and short, arising } \\
\text { from the underlying inflammatory vasculature, with a scattered distribution, } \\
\text { but in case of mature hyperplasia, the deeper inflammatory vascular network } \\
\text { is not visible, and only the elongated CLs can be easily seen. In the case of } \\
\text { vegetating keratosis, the deeper inflammatory vascular network is often not } \\
\text { visible, and the elongated CLs are difficult to see. A particular type of } \\
\text { "bobby-pin" can be seen in laryngeal papillomatosis, where we found the } \\
\text { typical papilla encasing the "bobby-pin" inside the papilloma. }\end{array}$ \\
\hline Type III & Mild-moderate dysplasia & $\begin{array}{l}\text { Vascular changes become progressively more consistent, with elongated small } \\
\text { vessels in the typical "bobby-pin" shape, but some arborescence appears at } \\
\text { the end of the CLs. }\end{array}$ \\
\hline Type IV & $\begin{array}{l}\text { High-grade dysplasia/carcinoma } \\
\text { in situ/invasive carcinoma }\end{array}$ & $\begin{array}{l}\text { The vascularity of the chorion is more evident, and CLs appear significantly } \\
\text { dilated, with various shapes and a wide range of vascular architectural } \\
\text { changes such as corkscrews or tree-like patterns. }\end{array}$ \\
\hline
\end{tabular}

CLs = capillary loops.

The use of contact endoscopy in otolaryngology was first described in 1995 by Andrea et al $(103,104)$, however, up to now, only a few groups have evaluated this technique for in vivo histology $(105,106)$. In fact, expansion of squamous epithelium from the vocal fold edges to the areas of columnar epithelium can be clearly visualized by contact endoscopy; hyperkeratosis (i.e. deposits of anuclear cells on the epithelial surface) or leukoplakia are clearly observed on contact endoscopy, and the grade of dysplasia that may occur in association with them is generally indicated by the impaired nucleus/cytoplasm ratio, nuclear hyperchromasia, and variation in the number and appearance of the nucleoli (107).

False negative results may occur in case of incomplete penetration of the stain throughout the epithelial thickness thus hindering the identification of the grade of dysplasia; in this setting the presence of secretion decreases stain penetration, while the secretion itself precludes any direct contact between the endoscope and the mucosa epithelium. Another situation that may influence the incidence of false negative is the diagnosis of carcinoma in situ. Carcinoma in situ is characterized by heterogeneity of the cell population; however, angiogenesis is not present because the tumor process does not cross the basement membrane. It is therefore difficult to differentiate carcinoma in situ from invasive carcinoma. The finding of angioneogenesis definitely indicates carcinoma, whereas its absence does not exclude the possibility of invasive carcinoma (i.e.: the diagnosis of carcinoma in situ cannot be made with certainty). Finally, the presence of abundant necrosis in case of large tumor mass could cause bleed when touched by the endoscope thus impairing tissue staining and analysis of cell population. 
For all these reasons, the reliability of contact endoscopy in literature is reported within $75 \%$ to 88\% (108-110).

In summary, CE can examine only limited cellular architecture of the epithelium. This is due to the poor penetration of methylene blue, which only dyes superficial layers and potential optical artifacts at high magnification due to glare from light reflected from cells that are not in focus (the focal distance of the endoscope is $80 \mathrm{~mm}$ at 603 magnification and $30 \mathrm{~mm}$ at 1503 magnification) (111). In specific, difficulties of interpretation of the vascular patterns increase in the follow-up of the upper aero-digestive tract (UADT) cancers due to the changes of the surrounding tissues as a consequence of previous treatments such as radiotherapy. In order to improve these lacks, Storz Professional Image Enhancement System (SPIES) (KARL STORZ GmbH \& Co., Tuttlingen, Germany) has been recently proposed as a novel digital technique providing specific color renderings that pronounce the spectral separation of the recorded broad visible spectrum within the high-definition camera system and it does not require a narrow band light source. SPIES enhances the appearance of the mucosal surface structures and subepithelial vasculature by selected wavelengths of light, providing in addition to the standard mode using white light (WL), five different defined spectral ranges (Clara, Clara1- Chroma, Chroma, Spectra A, Spectra B). The main target of analysis of these techniques is neoangiogenesis, a prerequisite for the progression of precancerous and cancerous lesions of the upper aero-digestive tract (UADT) (102). SPIES, as well as narrow band imaging (NBI), allow for the recognition of the superficial changes of neoangiogenesis, and they require a certain degree of experience to avoid false positives, because the typical mucosal "spots" are not univocal and are challenging for clinical interpretation. It appears to have good sensibility $(79.6 \%-94.7 \%)$, specificity $(81 \%-95.5 \%)$, and accuracy (88\%$94 \%$ ) in the larynx (105) for distinguishing between benign and malignant mucosal lesions.

\subsection{Autofluorescence}

Autofluorescence (AF) is defined as natural fluorescence emission of tissue arising from endogenous fluorophores after exposure and activation by radiation of a suitable wavelength. In its resting state, a fluorophore is at a stable energy level at which it does not fluoresce. When a fluorophore is illuminated, its electrons are promoted to a higher energy level. In this excited state, the fluorophore is unstable and will quickly revert to a slightly more stable lower energy level by releasing heat. To return to its baseline the fluorophore emits light. Since some energy has already been released as heat, the emitted light is of lower energy and longer wavelength than that of the illuminating light (112). Fluorophores are present at different concentrations in healthy and neoplastic laryngeal mucosa; for example, nicotinamide adenine dinucleotide (NADH) predominates within neoplastic cells in its dehydrogenated nonfluorescent form $(113,114)$. Several fluorophores are normally found within laryngeal mucosa including prophyrins, elastin, collagen, and NADH $(115,116)$.

Because each fluorophore has a specific wavelength at which its electrons are maximally excited, it is possible to target specific fluorophores, such as NADH, using an illuminating light of a single wavelength (monochromatic). The underlying fundamental principle of different fluorescence 
emission in AF is related to neoplasia-induced changes in terms of tissue morphology, optical properties, and concentration of endogenous fluorophores (117). Autofluorescence diagnosis is based on the ability of oxidised flavin mononucleotide (FMN) in the normal cells to emit green fluorescence when exposed to blue light. Nicotinamide adenine dinucleotide plus hydrogen $(\mathrm{NADH})$ and flavin adenine dinucleotide (FAD) are important intracellular fluorophores found in all tissue layers; their concentration is nearly 100 times lower in malignant tissue than in benign tissue (118), therefore, malignant cells do not have fluorescence to the same degree as benign cells (119). At present, autofluorescence endoscopy (AFE) is increasingly being used for early detection of malignant mucosal changes in diverse areas of localization and various medical specialties (120-121). It is argued that the advantage of AFE compared to conventional white light endoscopy is based on the fact that premalignant and malignant lesions might be differentiated clearly from normal tissue because of decreased AF.

In 1924, Policard (122) observed the ability of tissue to fluoresce under certain conditions. Alfano et al., in 1984 (123), reported the possibility of differentiating between healthy and malignant tissue by means of their fluorescent characteristics. In 1933, Sutro and Burmann described the phenomenon of the different fluorescences of normal and tumor tissue.

Harris et al. (124) first used autofluorescence to identify neoplastic cells within the laryngeal mucosa, he demonstrated in a pilot study of eight cases with carcinoma of the vocal folds concluded that the technique can increase the accuracy of staging of cancer of the larynx and allows earlier diagnosis of tumors and their recurrence. In the same year Chissov et al. (125) reached the same conclusion. Malzhan et al. (116) reported a sensitivity of $97.3 \%$ and specificity of $83.8 \%$ for this technique in detecting precancerous and cancerous lesions in 127 patients. Beneficial role for AF has been ask reported by other works $(126,127)$, even if it has been identified a high rate of false positive results, as well (128).

In summary, despite promising literature evidence for this technique, there are, however, several limitations to autofluorescence examination. First, the illuminating light does not penetrate through diseased epithelium $(119,129)$. As a result, epithelial hyperkeratosis may hide neoplastic changes within the basal mucosal layer (130). Second, granulation tissue and teleangiectasia produce a similar reduction in bright-green fluorescence, attributed to the absorbative properties of a heme molecule, which makes them indistinguishable from neoplasia by autofluorescence (116). Third, scar tissue, necrosis, and inflammation can unpredictably alter mucosal fluorescence $(116,126,129)$. Thus, several conditions in the larynx may produce false positive and false negative findings.

In conclusion, further studies to increase the sensitivity and specificity and investigation of technical solutions to reduce the number of false negative and false positive cases are required.

\subsection{Narrow band imaging (NBI)}

Narrow band imaging (NBI) is an optical image enhancement technology that enhances vessels in the surface of mucosa using the characteristics of the light spectrum (131). The NBI system consists of the same components as conventional videoendoscopic systems: a light source, a camera unit, and a camera head or chip-equipped videoendoscope. Additionally, the NBI system contains a special image processor and a lighting unit with special filters that narrow the frequency range of 
emitted light to $400-430 \mathrm{~nm}$ (centered at $415 \mathrm{~nm}$, blue) and 525-555 nm (centered at $540 \mathrm{~nm}$, green) bands. Since the blue light wavelength $(415 \mathrm{~nm})$ is absorbed by hemoglobin the capillary blood vessels are seen brown in the summary picture. Currently, available evidence indicates that NBI may be a promising approach in the diagnosis of laryngeal cancer. It allows to visualize the structure of the intraepithelial blood vasculature which cannot be seen with conventional white light (132). In fact, the emitted light has less penetration and less scattering and is highly absorbed in hemoglobin, thus enhancing the image resolution. The reflection is captured by a charge-coupled device chip (CCD), and an image processor creates a composite pseudocolor image, which is displayed on a monitor, enabling NBI to enhance mucosal contrast without the use of dyes (133). The detection of surface mucosal changes that are characteristic of neoplastic lesions (e.g., dysplasia, in situ carcinoma, and carcinoma), epithelial abnormalities (thickening and changes in the surface layer), and vascular changes can be best achieved with NBI.

In the NBI mode, normal laryngeal mucosa consists of submucosal vessels (appearing green) connecting with an arborescent vascular network (appearing dark brown). Abnormalities of these intraepithelial papillary capillary loop (IPCL), located beneath the basement membrane of epithelium, are usually classified in accordance with their shape changes; such changes have been found to predict the depth of superficial cancer invasion $(134,135)$.

Classification of laryngeal lesions has been reported in Figure 4.

Figure 4. Diagrams of microvasculature and endoscopic views of vocal folds, illustrating classification of intraepithelial papillary capillary loop features using narrow band imaging. Type I (A1): thin, oblique and arborescent vessels are interconnected and intraepithelial papillary capillary loops are almost invisible. Type II (B1): diameter of oblique and arborescent vessels is enlarged, and intraepithelial papillary capillary loops are almost invisible. Type III (C1): intraepithelial papillary capillary loops are obscured by white mucosa. Type IV (D1): intraepithelial papillary capillary loops can be recognized as small dots. Type Va (E1): intraepithelial papillary capillary loops appear as solid or hollow, with a brownish, speckled pattern and various shapes. Type Vb (F1): intraepithelial papillary capillary loops appear as irregular, tortuous, line-like shapes. Type Ve (G1): intraepithelial papillary capillary loops appear as brownish speckles or tortuous, line-like shapes with irregular distribution, scattered on the tumor surface. 


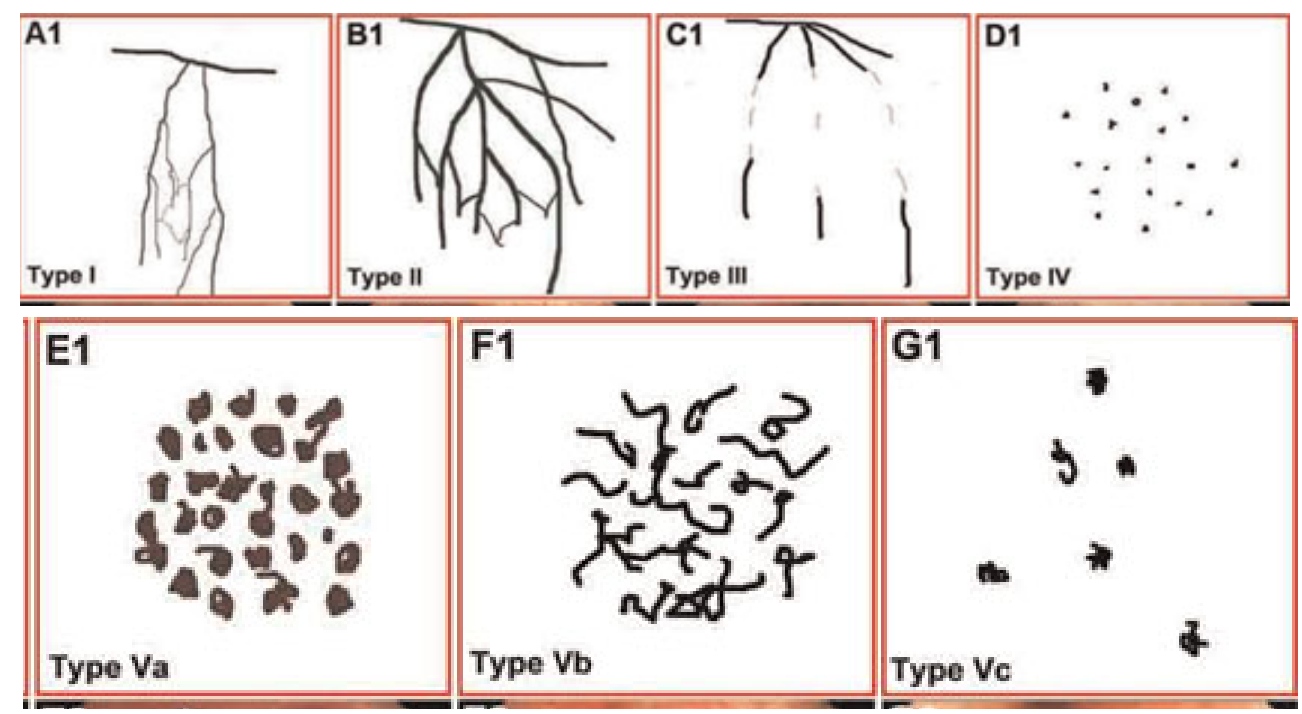

In type I, the intraepithelial papillary capillary loops are almost invisible, and oblique and arborescent vessels of small diameter can be clearly seen. In type II, the intraepithelial papillary capillary loops are also almost invisible, but the diameter of the clearly observed oblique and arborescent vessels is enlarged. In type III, the mucosa is white and the intraepithelial papillary capillary loops cannot be seen; if the white patch is thin, the oblique and arborescent vessels may be seen indistinctly, but if the white patch is thick the vessels will be obscured. In type IV, the mucosal intra- epithelial papillary capillary loops are visible with a relatively regular arrangement and low density, the capillary terminals are bifurcated or slightly dilated, and the intraepithelial papillary capillary loops appear as scattered, small, dark brown spots; the oblique and arborescent vessels are usually not visible.

Type $\mathrm{V}$ changes are subdivided into types $\mathrm{Va}, \mathrm{Vb}$ and $\mathrm{Vc}$ according to the shape, regularity and distribution of vessels. In type Va, intraepithelial papillary capillary loops are significantly dilated and of relatively high density, and appear to be solid or to have hollow, brownish, speckled features and various shapes. In type $\mathrm{Vb}$, the intraepithelial papillary capillary loop itself is destroyed, with its remnants presenting in a snake-, earthworm-, tadpole- or branch-like shape, and the microvessels are dilated, elongated and 'woven' in appearance. In type Vc, the lesion surface is covered with necrotic tissue, and the intraepithelial papillary capillary loops present as brownish speckles or tortuous shapes of uneven density which are irregularly scattered on the tumor surface.

Lesions viewed under NBI are usually recorded as: (1) malignant (i.e. type V); (2) suspected malignant (i.e. protuberant or ulcerative lesions covered with necrotic tissue, or leukoplakia of unknown type); or (3) benign (types I to IV) (135).

In the literature, there exist only a few publications on the exclusive use of NBI in laryngology (135-142). However, several studies assessed the value of NBI in the head and neck also including the larynx $(132,137,143-148)$.

NBI has shown in the larynx, over last years, improved sensitivity $61-91 \%$ and specificity $87-92 \%$ $(146,149)$ but false positives and false negatives have been reported, especially in the larynx, and increase with a short learning curve. 
This approach is promising for better discrimination of malignant and benign lesions as part of "prehistologic diagnosis" or "optical biopsy" (140). Nevertheless, there is no ultrathin, zooming, flexible videoendoscope available for ENT purposes; therefore, a combination of rigid telescopes and an HDTV camera head must be used to achieve sufficient resolution and ultrahigh magnification (150). Although most authors concur that NBI with or without HDTV is a valid diagnostic tool, this method has some limitations. The most relevant is certainly the possibility of generating, at least in the early phase of the learning curve, an increased number of false positives with consequently unjustified biopsies. This is mostly related to acute inflammation and chronic post-RT changes. Therefore, we believe that the incidence of false positive findings was mainly related to the experience of the examiners more than to specific mucosal changes.

\subsection{Ultrasound}

Over the past 15 years, there has been an effort by head and neck surgeons to bring optical imaging technology from the research laboratory into the operating theater to improve their ability to identify the tumor margin in vivo to guide surgical excision (112).

Sonography is regarded as the first imaging method routinely used for detecting cervical lymph node metastases from head and neck tumors $(151,152)$ but it has rarely been used as an imaging technique for investigation of the larynx. Previous studies in the literature have yielded promising results for high-frequency sonography in diagnosis of laryngeal carcinoma (153-155) However, the use of sonography in tumor staging is still limited.

Since the 1970s, B-mode US imaging has been used with some success in the laryngeal area to identify mass and cystic lesions at the vocal folds (156) and to detect vocal fold paralysis $(157,158)$; moreover, ultrasonography has been also used to evaluate laryngeal tumors in a few studies, and some of these have proved that ultrasonography could assist tumor staging in patients with advanced laryngeal cancer $(155,159,160)$.

It has been demonstrated that ultrasound has a similar role in the visualization of hypopharyngeal tumor to CAT (161), thus whether ultrasonography has similar ability in evaluating laryngeal cancer is worthy to be investigated. Thyroid cartilage, pre-epiglottic space, paraglottic space, thyroid and cervical soft tissues, lying in the larynx anteriorly or superficially, are easy to be imaged with a high-frequency probe. Commonly, these structures are isoechoic or hyperechoic, which provide a contrast with a hypoechoic invading tumor (159). Hence, a high sensitivity and specificity has been achieved by ultrasonography in the evaluation of involvement of these structures and reported in literature $(160,162,163)$.

For instance, Kraft et al. (164) established anatomical landmarks for laryngeal endosonography (see Table 8) in order to allow a correct interpretation of sonographic images of its structures, and its known capacity to visualize critical regions such as the pre-epiglottic and paraglottic space endoscopically. 
Table 8. Description of normal sonoanatomy of the larynx correlated to horizontal whole organ sections of scanned speciments.

\begin{tabular}{|c|c|}
\hline Anatomical structure & Endosonographic appearance \\
\hline Trachea & $\begin{array}{l}\text { Horseshoe shape; inner and outer perichondrium hyperechoic; elastic cartilage hypoechoic; } \\
\text { membranous portion and tracheal mucosa normoechoic with homogeneous echopattern }\end{array}$ \\
\hline Cricoid & $\begin{array}{l}\text { Closed ring, interrupted anteriorly by cricothyroid membrane in its upper part; inner and outer } \\
\text { perichondrium hyperechoic; elastic cartilage hypoechoic; ossified cartilage normoechoic to hyperechoic; } \\
\text { cricoidal mucosa normoechoic with homogeneous echopattern }\end{array}$ \\
\hline Thyroid & $\begin{array}{l}\text { Triangular shape, interrupted anteriorly by thyroid notch in its upper part; inner and outer perichondrium } \\
\text { hyperechoic; elastic cartilage hypoechoic; ossified cartilage normoechoic to hyperechoic }\end{array}$ \\
\hline Vocal fold & $\begin{array}{l}\text { Paraglottic space and vocal muscle hypoechoic (directly adjacent to thyroid); vocal ligament and overlying } \\
\text { mucosa hyperechoic (situated more medially to vocal muscle) }\end{array}$ \\
\hline Ventricular fold & $\begin{array}{l}\text { Connective tissue of ventricular fold and preepiglottic space hyperechoic with rough echopattern; } \\
\text { paraglottic space and laryngeal ventrical hypoechoic; arytenoid hyperechoic }\end{array}$ \\
\hline Epiglottis & $\begin{array}{l}\text { Inner and outer perichondrium hyperechoic; elastic cartilage hypoechoic; epiglottic mucosa normoechoic } \\
\text { with homogeneous echopattern }\end{array}$ \\
\hline
\end{tabular}

Therefore, endolaryngeal ultrasonography seems to be able to predict the exact extension of a laryngeal tumor before surgery is performed, and to assist in finding the best therapeutic solution for the patient (165).

Furthermore, given its noninvasive nature and minimal disturbance to normal voice production, medical US imaging should be an ideal tool for studying vocal fold vibration. However, only a few studies have used dynamic US imaging to investigate the vibrating vocal folds $(166,167)$. The body motion of the vocal fold, which is ruled by mechanical process described by Hirano $(168,169)$, presents a vibration frequency $(f)>70 \mathrm{~Hz}$ which is higher than the frame rate $\left(f_{s}\right)$ of dynamic Bmode US $(<50 \mathrm{~Hz})$. Under this condition, real-time dynamic motion pictures of vocal fold vibration cannot be obtained using B-mode imaging. If the phonation frequency is tuned to be close to an integer multiple of $f_{s}$, a slow motion montage of vocal fold vibration may be obtained with B-mode US imaging. Such motion pictures may provide valuable data for analyzing vocal fold vibration especially in the body. This unique method has been described for the first time by Tsai et al. (170) who first attempted to represent a dynamic B-mode image of vocal fold vibration.

In conclusion, endosonography of the larynx produces horizontal slice images comparable with computed axial tomography (CAT) or magnetic resonance imaging (MRI) scans but with a higher resolution (170). During the imaging process, it is essential to position the probe as centrally as possible, avoiding contact with the laryngeal wall, it allows to directly measure the antero-posterior and medio-lateral diameters of the laryngeal neoplasm. The orotracheal tube, together with laryngeal structures such as cricoid and thyroid cartilages, are useful anatomical landmarks. Hence, direct observation of sonographic images help in orientation, too.

\subsection{Computed axial tomography (CAT) and Magnetic resonance imaging (MRI)}

The radiologist makes a valuable contribution to the staging of laryngeal cancer and this has a direct influence on treatment planning. Since approaches with both computed axial tomography (CAT) and magnetic resonance imaging (MRI) are acceptable, the choice in practice depends on machine availability and local expertise as well as the ability of the patient to tolerate a prolonged MRI examination. Cross sectional imaging provides information on tumor volume, extension to and 
across the laryngeal ventricle, infiltration of pharynx, para-glottic, pre-epiglottic and extra-laryngeal spaces, all of which impact on the potential for voice conserving partial laryngectomies and response to radiotherapy. Together with an assessment of nodal and systemic metastases, this enables the radiologist to play an integral role in the multidisciplinary selection of treatment options.

In specific, radiologists have to describe if the clinical laryngeal lesions presents a deeper extension and if this extension affect main laryngeal structures. They do this by analyzing different parameters as following:

\section{- Relationship of the tumor to the ventricular complex.}

In order to define the tumor as supraglottic, glottic or subglottic the radiologist must determine the level of the ventricular complex (comprising the true cord, false cord and the intervening ventricle). These landmarks are essentially mucosal, and usually they are assessed endoscopically, however, sometimes a bulky tumor could hide its distal visualization. The superior aspect of the ventricular complex is defined by the superior margin of the arytenoids cartilages (Figure 5a). The inferior aspect of the ventricular complex is defined by the true cords; radiologically identified by the transition of para-glottic fat to soft tissue in the wall of the larynx. This soft tissue represents the thyroarytenoid muscle (Figure 5b). In addition, the true cord lies at the upper margin of the cricoid cartilage (at the level of the anteriorly pointing vocal processes of the arytenoid cartilages). The posterior commissure is seen between the arytenoids cartilages. The subglottic region has a characteristic appearance where the cricoid cartilage is seen as a complete ring providing the foundation of the laryngeal skeleton (Figure 5c) Any soft tissue at the subglottic level is abnormal. The presence of an enlarged delphian node anterior to the trachea also indicates subglottic involvement.

\section{- Involvement of the submucosal spaces.}

An understanding of the pre-epiglottic and para-glottic spaces allows the radiologist to predict and identify patterns of tumor spread which have a critical impact on therapeutic decisions. Submucosal involvement of the pre-epiglottic and para-glottic spaces is difficult to evaluate clinically and endoscopically. The fatty C-shaped pre-epiglottic space is bounded anteriorly by the thyroid cartilage and thyrohyoid membrane and posteriorly by the epiglottis and quadrangular membrane (Figure 5a).

Figure 5. Axial CT scan through (a) false cords, (b) true cords, (c) subglottis to illustrate normal anatomy. (a) The tip of the arytenoids cartilages (white arrowhead) indicates the level of the false cords (open arrowhead) and also the superior aspect of the ventricular complex. (b) The anteriorly pointing vocal processes of the arytenoids (arrow) and the thyroarytenoid muscle (white arrowhead) are effacing the para-glottic fat within the wall of the larynx. This defines the level of the true cords and also the inferior aspect of the ventricular complex. The anterior and posterior (open arrowhead) commissures should only be represented by a thin mucosal layer. (c) There should be no soft tissue seen internal to the ring of the cricoid cartilage at subglottic level. 

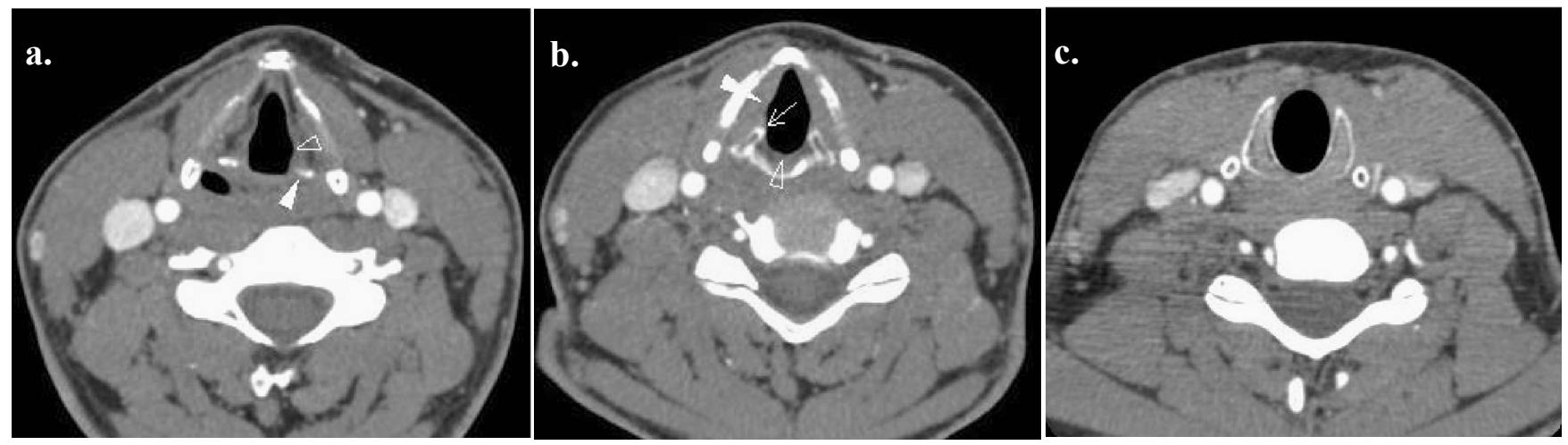

The pre-epiglottic space is continuous laterally with the paired para-glottic spaces (Figure 6).

Figure 6. The medial relations of the para-glottic fat (o) are seen to be the vestibule, the ventricle (long arrow) and the conus elasticus more inferiorly (curved arrow). Laterally it is related to thyroid cartilage and cricothyroid membrane. The true vocal cord is indicated (*).

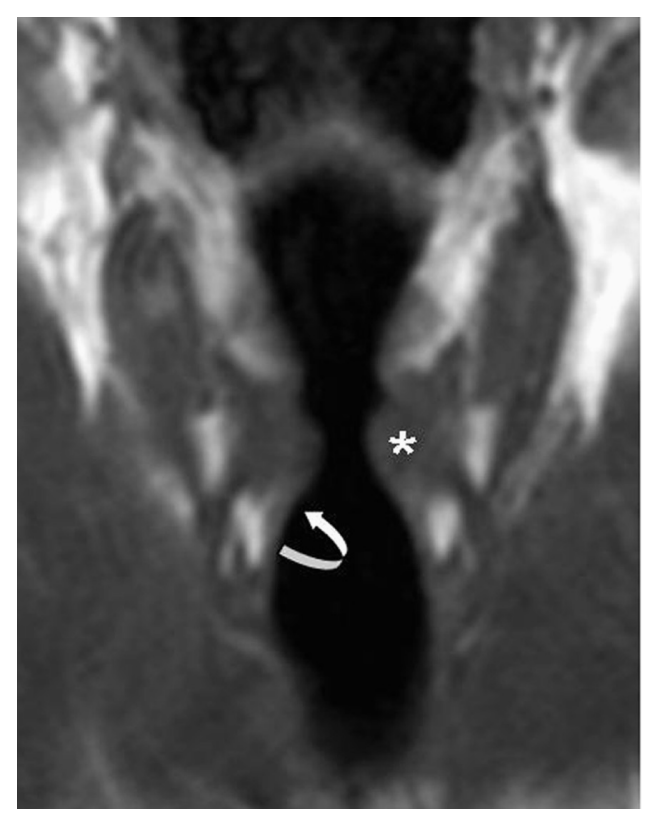

CT and MRI are 88-93\% accurate in the assessment of tumor extension within these spaces (171).

\section{- Anterior and posterior extension.}

In addition to defining the craniocaudal extension within the submucosal spaces relative to the ventricular complex landmarks, it is important to evaluate the anterior and posterior extension of tumor. This is particularly important at the level of the glottis, where usually glottic carcinoma, grows towards the anterior commissure which is frequently associated with thyroid cartilage involvement of the base of epiglottis (Figure 7). 
Figure 7. Axial CT scan shows thickening of the anterior commissure by glottic tumor (arrowhead).

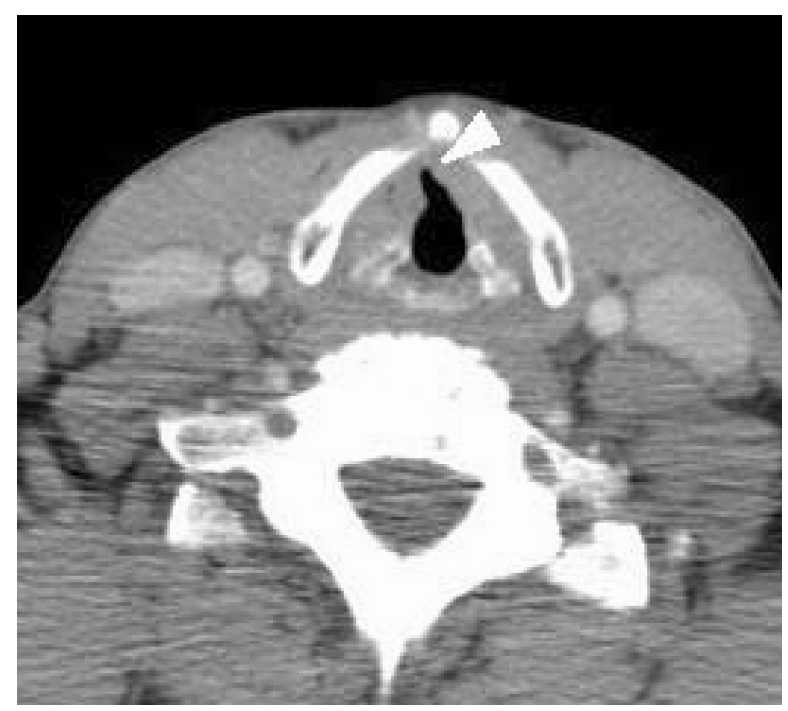

The cartilage is prone to invasion at this site because the internal perichondrium is deficient and the external perichondrium is thinner. The perichondrium covering the cartilage acts as a resistant barrier to invasion by tumor (171). It has been shown that cartilage invasion mainly occurs where the attachment of the collagen bundles interrupts the perichondrial barrier. As the cancer cells multiply, they separate the collagen bundles, forming linear passageways through the perichondrium. Thus, the sites of attachment of the strongest membranes, such as the anterior commissure tendon, are also the most frequent sites of invasion. Invasion of the cartilage framework invariably takes place at the site of ossified or calcified cartilage (172). Non-ossified cartilage is resistant to tumor infiltration due to its capacity to release proteins that inhibit tumor angiogenetic factor and collagenases (173). Recent published reports have shown that CT may yield acceptable sensitivity for detecting neoplastic invasion of laryngeal cartilage if the diagnostic criteria are selected and combined appropriately (174).

However, cartilage invasion is still sometimes overestimated, resulting in unnecessary total laryngectomies in some patients (175).

Previous studies using single-slice spiral CT scanners have concluded that the CT criteria used for determining neoplastic invasion of the thyroid cartilage include erosion, lysis, and transmural extralaryngeal tumor spread (176-180) (Figure 8).

Figure 8. Drawing to illustrate the criteria for evaluation of thyroid cartilage invasion. Erosion is defined as invasion beyond the inner cortex without reaching the outer cortex (less than half of the cartilage width), lysis is defined as almost reach- ing the outer cortex but with preservation of the cortex, and extra-laryngeal spread is defined as all-layer invasion through both the inner and outer cortex (penetration) of the cartilage, including the extra-laryngeal soft tissues (175). 


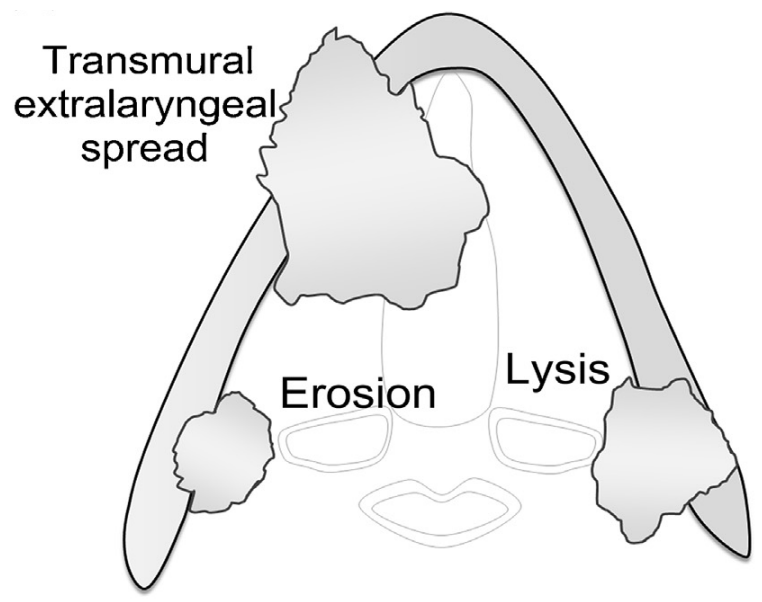

Tumor may also extend from the anterior commissure to an extra-laryngeal location caudal to the thyroid cartilage (via the cricothyroid membrane) and inferiorly to the subglottis. CAT is $75 \%$ accurate in predicting anterior commissure involvement (181). Predicting the presence of laryngeal cartilage invasion is a key aspect of the imaging assessment.

CAT and MRI are generally used to supplement microlaryngoscopy (MLS) in staging laryngeal cancer. Ideally, imaging should be able to predict the tumor size, the exact extension, a possible midline crossing, and cartilage infiltration in these lesions. Although CT proved to be helpful in the investigation of advanced malignancies (T2-T4), it often fails to show early cancer (Tis, T1 $<10$ $\mathrm{mm}$ ) due to the almost identical density of tumor tissue and the vocal muscle. With MRI, the same difficulties are encountered in demonstrating smaller tumors (Tis, T1 $<9 \mathrm{~mm}$ ), but the latter can better differentiate cancer from muscle tissue and depict invasion of the laryngeal framework (182). However, CT and MRI are expensive and cannot be performed during MLS. Further disadvantages are the radiation load of $\mathrm{CT}$, the long acquisition times for MRI, and the need for contrast agents in both methods.

\section{- CAT:}

CAT is the preferred imaging method for staging of laryngeal and hypopharyngeal cancer. Multislice CAT allows the radiologist to evaluate almost all the relevant imaging issues. Voltage of $120-140 \mathrm{kVp}$, tube current of at least $180 \mathrm{mAs}$, display matrix of $512 \times 512$ pixels and a collimation of approximately $1 \mathrm{~mm}$ should be used. A single bolus of contrast medium is effective, however a biphasic contrast enhancement protocol may be employed. The images are obtained with the patient supine and during quiet respiration (not while holding the breath). The neck should be in slight extension, and the head is aligned along the cephalocaudal axis to allow comparison of symmetrical structures. A longitudinal field of view extends from the skull base to the sterno-clavicular joints with the patient breathing quietly and not swallowing. Malpositioning may create an appearance that simulates disease. Every effort should be made to make the patient feel comfortable. It may be helpful to perform an additional examination focused on the tumor with e-phonation (for better assessment of the laryngeal ventricle, anterior commissure and aryepiglottic folds) or modified 
Valsalva (for better assessment of the priform sinus and post cricoid regions) manoeuvres (175). In addition to soft tissue windows, the bone windows (reconstructed with a bony algorithm) should be routinely evaluated if the tumor contacts ossified laryngeal cartilage. It should be ensured that axial images are reformatted in the plane of the larynx.

Spiral CAT is entirely dependent on the emergence of "slip ring" technology, which allows electrical energy to be transmitted to the gantry as it passes along the patient, allowing acquisition of CAT data in a helical fashion. It is also a faster process, with less discomfort to the patient and it is dynamic, with high resolution giving slices in continuity (183).

CAT provides a clearer illustration of bony structures and calcification than does MRI. Moreover, it is less expensive, faster and less susceptible to motion artifacts.Sclerosis is the most sensitive criterion but this often corresponds to reactive inflammation, particularly with respect to the thyroid cartilage. For instance, CAT signs of erosion and extra-laryngeal tumor of the thyroid, cricoid and arytenoid cartilages together with sclerosis of the cricoid and arytenoid cartilages result in a sensitivity of $64-72 \%$ and specificity of $86-94 \%$ for cartilage involvement, with an accuracy value of approximately $80 \%(177,184,185)$.

\section{- MRI:}

MRI is best performed with a high field MRI scanner. The MR examination requires a neck coil to obtain adequate resolution. Section thickness should be $4-5 \mathrm{~mm}$ for the neck coverage and 2-3 $\mathrm{mm}$ (0.1-0.2 mm interslice gap) for the focused study of the larynx. The display matrix size is $170 \times 256$ pixels minimum. A combination of T2-w sequences with fat saturation, T1-w sequences and T1-W fat saturated sequences with gadolinium, should be used in axial and coronal planes. The sagittal plane is also useful to assess potential tongue base involvement. Patients are asked to refrain from coughing and swallowing during the acquisition and MRI may produce poor results in the breathless or restless patient who is compromised by the tumor.

MR imaging is most frequently used if there is uncertainty in assessing cartilage involvement, when this is critical to therapeutic decisions. It may also better define the margin between the tumor and thyroarytenoid muscle and involvement of the tongue base.

MRI scanning has become increasingly involved as a sensitive imaging modality for detecting neoplastic involvement of the thyroid cartilage, primarily because of its high negative-predictive value. However, recently has been demonstrated MRI low positive-predictive value (68-71\%), due to its failure in distinguish edema and inflammation surrounding the tumor from the true cartilage invasion $(174,177,178)$. In addition, it could overestimate cartilage invasion (186). MRI criteria for cartilage involvement by tumor are high signal on fat suppressed T2-w images and/or enhancement on post gadolinium fat suppressed T1-w images in the cartilage adjacent to the tumor, or the presence of extra-laryngeal tumor. The most recent studies describing radiological-pathological correlation demonstrated slightly improved sensitivity of $89-95 \%$ and reduced specificity of $74-84 \%$ relative to CAT; but accuracy still demonstrates value of about $80 \%$ as CAT $(177,178,184,185)$. Moreover, MRI can be a lengthy and expensive procedure. Ana patient motion as simple as swallowing may generate significant artifacts. 
Moreover, high-resolution MRI had been used to construct a three-dimensional anatomical model of the cartilages of the human larynx (187), providing an anatomical framework for registering different larynges to the same coordinate space. Lately, a three-dimensional educational computer model of the larynx was developed from MRI scans (188). Two recent studies opened new directions for applying MRI for laryngeal imaging: MR microimaging of excised larynges confirmed valid high-resolution imaging of laryngeal tissue microstructure (189), and dynamic MRI of laryngeal and vocal fold vibrations was realized for the first time for measuring of laryngeal structures and glottal parameters in dynamic function (190).

\subsection{Positron emission tomography (PET, CT/PET)}

Fluorine-18 fluorodeoxyglucose (FDG) positron emission tomography (PET) plays an increasing roll in the assessment of head and neck cancer, both for primary staging and for post-therapy management (191). PET is a functional imaging modality assessing the metabolic status of tumors and has proved superior to CT and MRI in differentiating recurrence from post-radiation effects or scar in patients with carcinoma of the larynx (191,192). A negative PET scan excludes recurrence with a high certainty. Positive PET scan findings should be assessed with biopsy and follow up PET imaging if this is negative. It should be noted that physiological uptake of [ $\left.{ }^{18} \mathrm{~F}\right]$ fluorodeoxyglucose ( $\left.\left[{ }^{18} \mathrm{~F}\right] \mathrm{FDG}\right)$ is observed due to vocal cord activity, so a "silent protocol" should be employed. Recent advances in technology make it possible to fuse anatomical images with functional images. The use of combined 18F-FDG PET/CT (PET/CT) fusion images has been shown to improve diagnostic accuracy (1-3). For head and neck cancers, the reported sensitivity of PET/CT is $98 \%$, specificity of $92 \%$, with an accuracy of $94 \%$ for the identification of a malignancy of the head and neck; this is a higher accuracy than with PET or CT alone (193). Due to its high costs its use is advocated in case of uncertain results of CAT or MRI, and to complete pretreatment neck staging or in case of suspect for post-radiotherapy recurrence.

\section{Objective of the review.}

The clinical diagnosis of laryngeal pathology is important, as this primarily determines the next step towards the treatment. Unfortunately, it is not uncommon for different clinicians to use different nomenclature or to identify different stage for the same laryngeal lesion. This obviously makes evaluation and comparison among specialists indications difficult, and it does affect the treatment. In order to make this inter-observer variability as much lower as possible, attempts should be made to improve diagnostic technology, stricter/more universally accepted definitions and supervised training of junior doctors in a voice clinic environment. This should in turn lead to useful outcome data and treatment recommendations.

The objectives of this systematic review and meta-analysis were to synthesize the key diagnostic management for laryngeal lesions through a review of the latest advances in laryngeal imaging modalities published in the past 10 years. 


\section{Methods.}

The systematic review was performed using independently developed search strategies by the first author in literature review methodology. The databases interrogated included PubMed Clinical Queries www.ncbi.nlm.nih.gov. Reference lists from identified articles were searched and crossreferenced to identify additional relevant articles, which we also include in this review, and relevant opinion leaders were contacted.

The search terms included the following various combinations to maximize the yield: larynx, laryngeal precancerous lesions, laryngeal cancer, white light endoscopy (WLE), stroboscopy, contact endoscopy (CE), autofluorescence endoscopy (AFE), ultrasound (US), narrow band imaging (NBI), computed axial tomography (CAT), magnetic resonance imaging (MRI), positron emission tomography (PET-CAT/PET).

The search was performed for the first time on January 2014 and was set to automatically update periodically until April 2015.

First, duplicates were removed electronically. Then, abstracts were reviewed to exclude obviously irrelevant articles. Experimental studies and papers dealing with pathologies other than precancerous and cancerous lesions of the larynx were excluded.

The inclusion criteria were deliberately kept wide to encompass as many articles as possible without compromising the validity of the results, and they are listed in Table 9.

Table 9. Inclusion criteria list.

\begin{tabular}{|l|l|}
\hline & Inclusion criteria for paper selected for the review \\
\hline 1 & Articles published from 2005 onward; \\
\hline 2 & Published series of $\geq 10$ patients; \\
\hline 3 & Sensitivity and specifity clearly shown or detachable; \\
\hline 4 & Clear description of selection criteria; \\
\hline 5 & $\begin{array}{l}\text { Diagnostic methods including: ultrasound analysis, autofluorescence and fluorescent } \\
\text { analysis, contact endoscopy, endoscopy, stroboscopy, narrow band imagind (NBI), } \\
\text { computed axial tomography (CAT), magnetic resonant imaging (MRI), positron emission } \\
\text { tomography (PET); }\end{array}$ \\
\hline
\end{tabular}

We filtered the studies to ensure that only data from centers that had published on at least 10 patients were included in the review; this was done as a quality assurance measure as there are several case series in the literature, which have published the results of small numbers of cases spanning several years. Non-English language papers and duplicates were excluded.

The search excluded articles that were published before 2005 because we believe that it was pragmatic to include articles that were published on each diagnostic technique at least in the last decade, allowing time for the learning curve to be climbed for method such as narrow band imaging (NBI), came into clinical practice since 1997, and in order to try to compare data as much more 
recent as possible, due to the latest technology progression and the entrance in usage of more advanced instruments as magnetic resonance imaging (MRI) with high-resolution or diffusionweighted imaging (DWI) MRI.

All data were independently extracted by two authors and quality assessed. Eligibility for inclusion was separately assessed and when in doubt discussed and decided by consensus.

A first qualitative and descriptive review-analysis of selected articles was carried on; whilst, exclusively, publications comparing diagnostic imaging findings with definitive histopathology results were included in our meta-analysis. For articles not reporting raw data, letters were sent to the corresponding authors requesting them, otherwise they were excluded from the quantitative analysis.

Raw data from the meta-analysis were entered into the appropriate contingency tables to allow calculation of sensitivity, specificity, accuracy, Jounden's index, positive and negative predictive values for each diagnostic imaging technique.

In accordance with the literature, malignant (i.e., carcinoma in situ, invasive cancer) were classified as positive whereas premalignant lesions (i.e., moderate and severe dysplasia) were classified as negative; benign lesions (inclusive of simple hyperplasia and/or mild dysplasia) were calculated as negative, as well.

Similar analyses were performed for the procedure type. Each diagnostic technique was evaluated for its capacity in distinguish premalignant from cancerous laryngeal lesions; when possible, every method was analyzed for its accuracy in identifying advanced stage cancers from early stages.

For this reason, in order to fulfill the need to identify the best diagnostic technique for every diagnostic step, we made two different meta-analysis:

1. On articles reporting diagnostic techniques able to make a differential diagnosis between preneoplastic and cancerous lesions;

2. On those diagnostic methods that allow a more accurate analysis of tumor's volume and extension in order to refine the staging phase.

\subsection{Study characteristics and quality assessment}

To our knowledge, no widely accepted measures of quality assessment of case series exist; here, all included papers were graded using the NICE scoring scale for retrospective case series (Available at:http://www.nice.org.uk/nicemedia/pdf/Appendix_04_qualityofcase_series_form_preop.pdf). This is a scoring scale with eight items, with each item scoring zero or one based on the study methods (Yes $=1 ; \mathrm{No}=0$ ). Scores of $\geq 6$ are considered to indicate a good quality study, scores between four and five as fair and those studies with a score of three are treated as poor quality (Table 10). 
Table 10. Quality assessment scale.

\begin{tabular}{|l|l|}
\hline & Quality assessment for case series \\
\hline 1 & Case series collected in more than 1 center (i.e.: a multicentric study) \\
\hline 2 & Is the hypothesis/aim/objective of the study clearly described? \\
\hline 3 & Are the inclusion and exclusion criteria (case definition) celarly reported? \\
\hline 4 & Is there a clear definition of the outcomes reported? \\
\hline 5 & Were data collected prospectively? \\
\hline 6 & Is there an explicit statement that patients were recruited consecutively? \\
\hline 7 & Are the main findings of the study clearly described? \\
\hline 8 & Are outcomes stratified (i.e.: by disease stage, abnormal test results, patient characteristics)? \\
\hline
\end{tabular}

\subsection{Statistics}

Fisher's exact test was used for statistical analysis of categorical data for the descriptive review, and a value of $\mathrm{p}<.05$ was considered significant.

Then, all the included studies for the meta-analysis, provided sufficient data (true-positive [TP], false-positive [FP], true-negative [TN], and false-negative [FN]) to permit calculation of sensitivity (Se) and specificity (Sp). Sensitivity and specificity at a 95\% confidence interval (CI) were then pooled using a bivariate regression approach (194)

Data were abstracted into Microsoft Excel (Microsoft Corporation, One Microsoft Way Redmond, WA 98052-6399) and statistical package R version 2.9 was used for all analyses (The R Foundation for Statistical Computing, Vienna, Austria).

We constructed hierarchical summary receiver operating characteristic (SROC) curves to assess the interaction between sensitivity and specificity. The areas under the ROC curves (AUCs) were used to analyze the diagnostic precision of each diagnostic technique for differentiation of precancerous lesions from cancers and for distinguish advanced star tumors from early stage cases.

Thereafter, the effect of publication and other bias was assessed visually using a funnel plot, and the $I^{2}$ statistic was calculated (195). This is a measure of heterogeneity between the studies and ranges from $0 \%$ to $100 \%$; high figures indicate greater heterogeneity in the data.

When studies have low heterogeneity (pragmatically, $I^{2}<25 \%$ ), the differences between reported outcomes can be explained simply by the observed natural differences between patients. In this case we can consider that all patients are part of the same larger pool. A fixed-effects meta-analysis is appropriate in which each patient is given approximately equal weight. However, with high heterogeneity, the studies differ by more than can be explained by intrapatients effects. This implies that there were differences in the patients studies, in the treatment interventions, or in the outcome measures. In this case, a random-effects meta-analysis is appropriate in which each study is given more equal weight. 


\section{Results.}

The search strategy identified 7215 articles in Medline from 1950s to the search date. 3616 articles published after the 2005 were selected, imported into Endnote, and the duplicates were removed. The removal of duplicates, non-English language works and of articles about other items rather than precancerous and cancerous laryngeal lesions yielded a total of 214 publications.

These 214 publications were considered for the qualitative systematic review of the literature, on the other hand, only 41 out of 241 were selected for the meta-analysis.

The various stages of systematically assessing the abstracts and reasons for exclusion from the review are described in Figure 9. 
Figure 9. This flow chart illustrates the process that was used to select articles for the review.

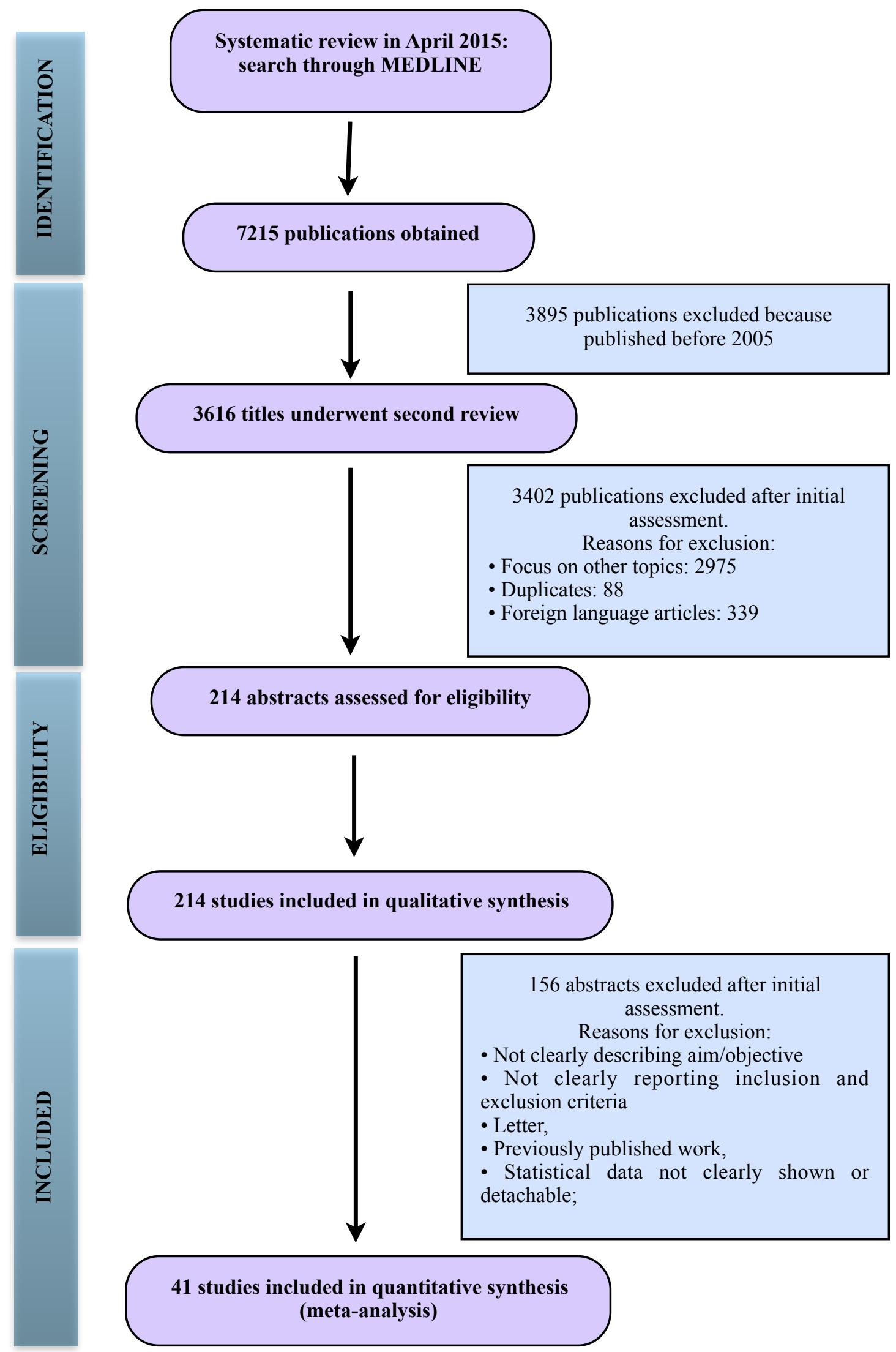


23 articles (I group) out of 41 were addressed towards the differential diagnosis among precancerous and cancerous lesions, whilst the remaining 18 articles (II group) were analyzed for the assessment of the identification of advanced stage cancers reporting cartilage involvement against the diagnosis of early staged laryngeal tumors.

Quality score assessment and each article clinical characteristics selected for the meta-analysis are summarized in Table 11 and 12.

Table 11. Various contributions of the article selected for the meta-analysis addressed towards the definition of the diagnostic precision of white light endoscopy (WLE) (yellow), stroboscope (white), contact endoscopy (CE) (light blue), autofluorescence endoscopy (AFE) (grey), narrow band imaging (NBI) (red), computed axial tomography (CTA) (orange), magnetic resonance imaging (MRI) (green) for laryngeal cancer against precancerous lesions.

(Some articles analyzed more than one system and they are indicated by ${ }^{*}$ or ${ }^{\circ}$;).

\begin{tabular}{|c|c|c|c|c|c|c|c|}
\hline Author & $\begin{array}{l}\text { Quality } \\
\text { score }\end{array}$ & $\begin{array}{l}\text { Number of } \\
\text { study years }\end{array}$ & $\begin{array}{l}\text { Number } \\
\text { of } \\
\text { patients }\end{array}$ & $\begin{array}{c}\text { Number } \\
\text { of } \\
\text { diseased } \\
\text { patients }\end{array}$ & $\begin{array}{l}\text { Sensitivity } \\
\quad \text { (Se) }\end{array}$ & $\begin{array}{l}\text { Specificity } \\
\text { (Sp) }\end{array}$ & $\begin{array}{l}\text { Younden's } \\
\text { Index (J) } \\
(\mathrm{Se}+\mathrm{Sp}-1)\end{array}$ \\
\hline $\begin{array}{c}\text { Bertino G. et al, } \\
2015^{\wedge \wedge \wedge}\end{array}$ & 5 & 2 & 217 & 143 & $98.7 \%$ & $33 \%$ & 0.20 \\
\hline Kraft M et al., 2014* & 6 & 1 & 205 & 57 & $79 \%$ & $95 \%$ & 0.74 \\
\hline Dobre $M$ et al., $2014^{* *}$ & 5 & 1 & 56 & 16 & $85 \%$ & $75 \%$ & 0.06 \\
\hline $\begin{array}{l}\text { Caffier PP et al, } \\
2013 * * *\end{array}$ & 7 & 1 & 32 & 17 & $100 \%$ & $94 \%$ & 0.94 \\
\hline $\begin{array}{c}\text { Shang DS et al., } \\
2013^{* * * *}\end{array}$ & 6 & 2 & 50 & 33 & $81.8 \%$ & $64.7 \%$ & 0.46 \\
\hline Crosetti E et al., $2012^{\circ}$ & 7 & 2 & 140 & 62 & $85.0 \%$ & $40.0 \%$ & 0.25 \\
\hline Piazza C et al., $2011^{\circ \circ}$ & 5 & 2,5 & 444 & 279 & $41 \%$ & $92 \%$ & 0.33 \\
\hline Ni XG et al., $2011^{\circ 0 \circ}$ & 7 & 0.5 & 85 & 45 & $68.9 \%$ & $90 \%$ & 0.59 \\
\hline Baletic N., 2010 & 5 & 1 & 45 & 37 & $73 \%$ & $98 \%$ & 0.71 \\
\hline Piazza C et al., $2010^{\wedge}$ & 4 & 1 & 279 & 110 & $33.0 \%$ & $95.0 \%$ & 0.28 \\
\hline Watanabe et al., $2008^{\wedge \wedge}$ & 6 & 1 & 667 & 221 & $51.1 \%$ & $99.7 \%$ & 0.51 \\
\hline Saetti R et al., 2007^^^ & 6 & 2 & 46 & 23 & $57.1 \%$ & $84.8 \%$ & 0.42 \\
\hline Uloza V et al., 2013 & 6 & 2 & 159 & 34 & $85.3 \%$ & $85.3 \%$ & 0.60 \\
\hline Puxeddu et al., 2015 & 4 & 0,5 & 44 & 21 & $97.6 \%$ & $97.6 \%$ & 0.95 \\
\hline Warnecke A et al., 2010 & 7 & 1 & 42 & 10 & $90.0 \%$ & $94 \%$ & 0.84 \\
\hline Cikojevic D et al., 2008 & 5 & 1 & 142 & 49 & $79.59 \%$ & $100 \%$ & 0.79 \\
\hline Dobre M, 2014** & 5 & 1 & 56 & 16 & $92 \%$ & $87 \%$ & 0.08 \\
\hline Kraft M et al., 2014* & 6 & 1 & 205 & 57 & $91 \%$ & $84 \%$ & 0.75 \\
\hline Succo G., 2014 & 4 & 4 & 286 & 73 & $96.5 \%$ & $98.5 \%$ & 0.95 \\
\hline $\begin{array}{l}\text { Caffier PP et al., } \\
2013^{* * *}\end{array}$ & 7 & 1 & 32 & 17 & $94 \%$ & $69 \%$ & 0.63 \\
\hline Crosetti E et al., $2012^{\circ}$ & 7 & 2 & 140 & 62 & $86.6 \%$ & $41 \%$ & 0.27 \\
\hline
\end{tabular}




\begin{tabular}{|c|c|c|c|c|c|c|c|}
\hline Author & $\begin{array}{l}\text { Quality } \\
\text { score }\end{array}$ & $\begin{array}{l}\text { Number of } \\
\text { study years }\end{array}$ & $\begin{array}{c}\text { Number } \\
\text { of } \\
\text { patients }\end{array}$ & $\begin{array}{c}\text { Number } \\
\text { of } \\
\text { diseased } \\
\text { patients }\end{array}$ & $\begin{array}{l}\text { Sensitivity } \\
\text { (Se) }\end{array}$ & $\begin{array}{l}\text { Specificity } \\
\text { (Sp) }\end{array}$ & $\begin{array}{l}\text { Younden's } \\
\text { Index (J) } \\
(\mathrm{Se}+\mathrm{Sp}-1)\end{array}$ \\
\hline Baletic N., $2010^{\circ 000}$ & 5 & 1 & 45 & 37 & $89 \%$ & $78 \%$ & 0.67 \\
\hline Saetti R et al., $2007^{\wedge \wedge \wedge}$ & 6 & 2 & 46 & 23 & $94.3 \%$ & $91.3 \%$ & 0.85 \\
\hline Arens $C$ et al., 2006 & 6 & 1,5 & 42 & 30 & $97 \%$ & $82 \%$ & 0.79 \\
\hline $\begin{array}{l}\text { Bertino G. et al., } \\
2015^{\wedge \wedge \wedge}\end{array}$ & 5 & 2 & 217 & 143 & $97.4 \% \%$ & $85 \%$ & 0.82 \\
\hline Lukes P et al., 2014 & 5 & 3 & 109 & 43 & $100 \%$ & $82 \%$ & 0.82 \\
\hline $\begin{array}{l}\text { Zobrodsky M et al., } \\
2014\end{array}$ & 4 & 4 & 66 & 14 & $92 \%$ & $76 \%$ & 0.68 \\
\hline Kraft $M$ et al., 2014* & 6 & 1 & 205 & 57 & $97 \%$ & $96 \%$ & 0.93 \\
\hline Piazza C et al., $2011^{\circ 00}$ & 5 & 2,5 & 444 & 279 & $97 \%$ & $84 \%$ & 0.81 \\
\hline Ni XG et al., $2011^{\circ 00}$ & 7 & 0.5 & 85 & 45 & $89 \%$ & $93.2 \%$ & 0.82 \\
\hline Irjala H. et al., 2011 & 4 & 0.12 & 73 & 35 & $55 \%$ & $98 \%$ & 0.53 \\
\hline Piazza C et al., $2010^{\wedge}$ & 4 & 1 & 279 & 110 & $98.0 \%$ & $90.0 \%$ & 0.88 \\
\hline Watanabe et al., 2009 & 6 & 1 & 34 & 23 & $91,30 \%$ & $91,60 \%$ & 0.83 \\
\hline Watanabe et al., $2008^{\wedge \wedge}$ & 6 & 1 & 667 & 221 & $97.7 \%$ & $98.9 \%$ & 0.97 \\
\hline Beser M et al., 2009 & 7 & 1 & 38 & 11 & $88 \%$ & $50 \%$ & 0.38 \\
\hline $\begin{array}{l}\text { Shang DS et al., } \\
2013^{* * * *}\end{array}$ & 6 & 2 & 50 & 33 & $91 \%$ & $76.5 \%$ & 0.67 \\
\hline
\end{tabular}

Table 12. Various contributions of the article selected for the meta-analysis addressed towards the definition of the diagnostic precision of ultrasound (US) (yellow), computed axial tomography (CTA) (orange), magnetic resonance imaging (MRI) (green) and position emission tomography (PET-CAT/PET) (purple) for advanced laryngeal tumors with cartilage involvement against early staged cancers.

(Some articles analyzed more than one system and they are indicated by * or ${ }^{\circ}$;)

\begin{tabular}{|c|c|c|c|c|c|c|c|}
\hline Author & $\begin{array}{l}\text { Quality } \\
\text { score }\end{array}$ & $\begin{array}{l}\text { Number of } \\
\text { study years }\end{array}$ & $\begin{array}{c}\text { Number } \\
\text { of } \\
\text { patients }\end{array}$ & $\begin{array}{c}\text { Number } \\
\text { of } \\
\text { diseased } \\
\text { patients }\end{array}$ & $\begin{array}{l}\text { Sensitivity } \\
\text { (Se) }\end{array}$ & $\begin{array}{l}\text { Specificity } \\
\text { (Sp) }\end{array}$ & $\begin{array}{l}\text { Younden's } \\
\text { Index (J) } \\
(\mathrm{Se}+\mathrm{Sp}-1)\end{array}$ \\
\hline Xia CX et al., 2013* & 6 & 3 & 79 & 40 & $91.6 \%$ & $95.6 \%$ & 0.87 \\
\hline Hu Q et al., 2012** & 6 & 1 & 36 & 26 & $88.6 \%$ & $85.5 \%$ & 0.74 \\
\hline Kraft M et al., 2013*** & 7 & 1 & 760 & 308 & $84 \%$ & $93 \%$ & 0.77 \\
\hline $\begin{array}{l}\text { Allegra E et al. } \\
2014^{* * * *}\end{array}$ & 6 & 2 & 20 & 4 & $50 \%$ & $100 \%$ & 0.50 \\
\hline Han MW et al. 2013 & 5 & 12 & 32 & 21 & $57 \%$ & $94 \%$ & 0.51 \\
\hline Hartl DM et al. 2013 & 6 & 16 & 236 & 19 & $10.5 \%$ & $94 \%$ & 0.04 \\
\hline Kraft M et al. $2013 * * *$ & 7 & 1 & 510 & 228 & $68 \%$ & $84 \%$ & 0.52 \\
\hline Xia CX et al. 2013* & 6 & 3 & 79 & 40 & $72.7 \%$ & $66.7 \%$ & 0.40 \\
\hline Hu Q et al. $2012 * *$ & 6 & 1 & 36 & 26 & $82.9 \%$ & $91.6 \%$ & 0.74 \\
\hline
\end{tabular}




\begin{tabular}{|c|c|c|c|c|c|c|c|}
\hline Author & $\begin{array}{l}\text { Quality } \\
\text { score }\end{array}$ & $\begin{array}{l}\text { Number of } \\
\text { study years }\end{array}$ & $\begin{array}{l}\text { Number } \\
\text { of } \\
\text { patients }\end{array}$ & $\begin{array}{c}\text { Number } \\
\text { of } \\
\text { diseased } \\
\text { patients }\end{array}$ & $\begin{array}{l}\text { Sensitivity } \\
\text { (Se) }\end{array}$ & $\begin{array}{l}\text { Specificity } \\
\text { (Sp) }\end{array}$ & $\begin{array}{l}\text { Younden's } \\
\text { Index (J) } \\
(\mathrm{Se}+\mathrm{Sp}-1)\end{array}$ \\
\hline Celebi I et al. 2012 & 6 & 1 & 27 & 18 & $88.9 \%$ & $88.9 \%$ & 0.77 \\
\hline Beitler JJ et al. 2010 & 7 & 10 & 104 & 41 & $49 \%$ & $92 \%$ & 0.41 \\
\hline Tino Just et al 2010 & 5 & 2 & 35 & 15 & $87 \%$ & $95 \%$ & 0.82 \\
\hline Beser et al. 2009 & 6 & 1 & 38 & 26 & $88 \%$ & $50 \%$ & 0.38 \\
\hline Jeong HS et al. 2008 & 7 & 2 & 114 & 55 & $82.5 \%$ & $82.7 \%$ & 0.65 \\
\hline de Souza RP et al. 2007 & 7 & 5 & 60 & 14 & $100 \%$ & $93.5 \%$ & 0.93 \\
\hline Fernandes R et al. 2006 & 5 & 1 & 27 & 15 & $80 \%$ & $92 \%$ & 0.72 \\
\hline Gordin A et al. $2006^{\circ}$ & 6 & 2 & 51 & 25 & $88 \%$ & $38 \%$ & 0.04 \\
\hline $\begin{array}{l}\text { Allegra E et al. } \\
2014 * * * *\end{array}$ & 6 & 2 & 20 & 4 & $100 \%$ & $100 \%$ & 1 \\
\hline Banko B et al. 2014 & 6 & 2 & 40 & 19 & $79 \%$ & $47 \%$ & 0.26 \\
\hline Kraft M et al. $2013 * * *$ & 7 & 1 & 150 & 70 & $63 \%$ & $89 \%$ & 0.52 \\
\hline Kinshuck AJ et al. 2012 & 5 & 10 & 31 & 22 & $64 \%$ & $67 \%$ & 0.31 \\
\hline Banko B et al. 2011 & 7 & 1 & 34 & 5 & $100 \%$ & $93 \%$ & 0.93 \\
\hline Becker M et al. 2008 & 6 & 1 & 121 & 49 & $96 \%$ & $75 \%$ & 0.71 \\
\hline J.Wedman et al. 2009 & 4 & 1 & 10 & 7 & $90 \%$ & $85 \%$ & 0.75 \\
\hline Gordin A et al. $2006^{\circ}$ & 6 & 2 & 112 & 66 & $96 \%$ & $61 \%$ & 0.57 \\
\hline
\end{tabular}

\section{- I group articles: cancerous vs. precancerous lesions assessment.}

The I group of articles reported an equal number of good (12) and fair (11) quality, in terms of determination of laryngeal cancerous lesions against precancerous lesions, a mean value for Se of $84.425 \pm 19.098$ SD (95\% CI 78.640-90.210, median 90.5\%) together with a similar mean value of $83.031 \pm 17.559 \mathrm{SD}$ for $\mathrm{Sp}(95 \%$ CI $77.089-88.972$, median $88.5 \%)$. These results are referring to a good pool of laryngeal lesions analyzed (3301) with a total amount of 1386 cancerous lesions identified overall the studied populations. Any statistical significance was found among continuos data $(\mathrm{p}=0.7818)$.

In specific, results about WLE precision on identify and distinguish superficial cancerous lesions from superficial precancerous lesions reported a very high $I^{2}$ value for both Se and Sp $(81.38 \%$ and $70.82 \%$, respectively), but with a AUC value of 91\% (Figure 10). 
Figure 10. SROC curve from bivariate model of WLE for cancerous lesions vs. precancerous lesions identification.

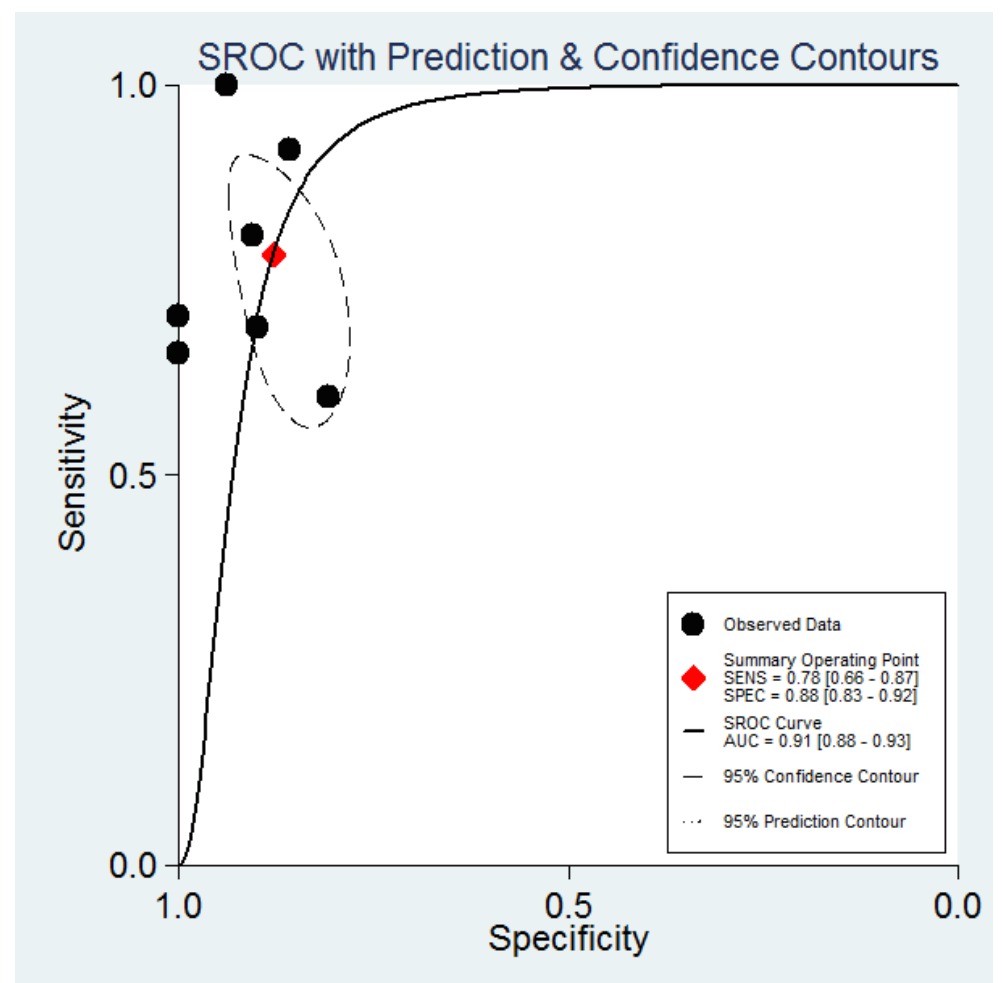

In regard to the diagnostic utility of WLE, Caffier et al. (196) showed a higher accuracy for WL (97\% vs. $81 \%$ ) than AFE and AFE data analysis provided a reduction in specificity of $25 \%$ in comparison to WLS, together with a high false positive value $(31 \%)$. These findings were in accordance with literature reports (197,198). On the other hand, even if Crosetti et al (199) confirmed that sensitivity and specificity of AFE in distinguish between benign and dysplastic/ neoplastic lesions are negatively impacted by different features (i.e.: hypertkeratosis-leucoplakia, abnormal hyperplasia, hypervascularized lesions, chronic laryngitis and lesions with bacterial infections or previously treated by surgery or radiotherapy), their results were completely different from those reported by Caffier et al. (196), indicating a very low accuracy in diagnostics for both these single used methods. They concluded supporting their hypothesis that a combination of both diagnostic tools allows a rise in sensitivity and specificity of about more than $35 \%$ and $25 \%$, respectively. Thus, they proposed to combine endoscopic tools through a multistep system, in order to obtain a greater sensitivity and specificity, to improve definition in direct microlaryngoscopy and in follow-up searching for synchronous/metachronous tumors.

Only one full text article were selected for the qualitative and quantitative analysis of the stroboscope diagnostic methods, confirming the lack of a statistical and accurate analysis of this technique in literature. Uloza et al. (200) found moderate-to-high rates of sensitivity (55.9- 85.3\%) and moderate rates of specificity $(51.1-60.0 \%)$ of the basic VLS parameters discriminating laryngeal carcinoma and other mass lesions of vocal folds. However, optimum system of four following VLS variables - regularity, mucosal wave on the affected side, symmetry of vibration, 
and symmetry of glottal image - increased the sensitivity up to $85.3 \%$ and specificity up to $74.4 \%$, and a final $\mathrm{J}$ index of 0.60 .

Few studies on CE were selected, as well. A recent article published by Puxeddu et al. (102) observed an accuracy of CE in the differential diagnosis between normal tissue and dysplasia versus carcinoma of $97.6 \%$. Warnecke and colleagues (106) declared a sensibility and specificity achieved by $\mathrm{CE}$ of $90 \%$ and $93.75 \%$, respectively. But among these, only one study compared contact endoscopy with frozen section histopathology in 142 patients with lesions of the larynx, where CE yielded a sensitivity of $79.59 \%$ and a specificity of $100 \%$, with a $J$ index of 0.79 (107). In summary we obtained a mean value of $89.0633 \pm 9.0415 \mathrm{SD}$ (95\% CI 66.6031-111.5236, median 90\%) for Se and of $97.200 \pm 3.020 \mathrm{SD}(95 \%$ CI 89.689-104.702, median 97.6\%) for Sp, with a p value of 0.3992, showing anyway a higher NPV (97\%) than PPV (82\%) for CE.

About 10 years later the introduction of $\mathrm{CE}$ into the clinical practice, few publications about autofluorescence in laryngology came out; it was 1995 when Harries et al. (124) demonstrated through their pilot in vivo study the autofluorescence properties of the laryngeal tissue and the potential accuracy of this system of performing earlier diagnosis and staging of laryngeal tumors. Considering at least one decade to allow the flatting of the learning curve, our selected articles showed values of Se and Sp of $92.550 \pm 3.611$ SD (95\% CI 89.531-95.569, median 93\%) and of $78.850 \pm 17.624$ SD (95\% CI 64.116-93.584, median 83\%), respectively; with a not quite statically significant $p$ value of 0.0583 . We constructed hierarchical SROC curve to assess the interaction between Se and Sp and these were $93 \%$ and $80 \%$, respectively with a AUC of $94 \%$. (Figure 11 ).

Figure 11. SROC curve from bivariate model of AFE for cancerous lesions vs. precancerous lesions identification.

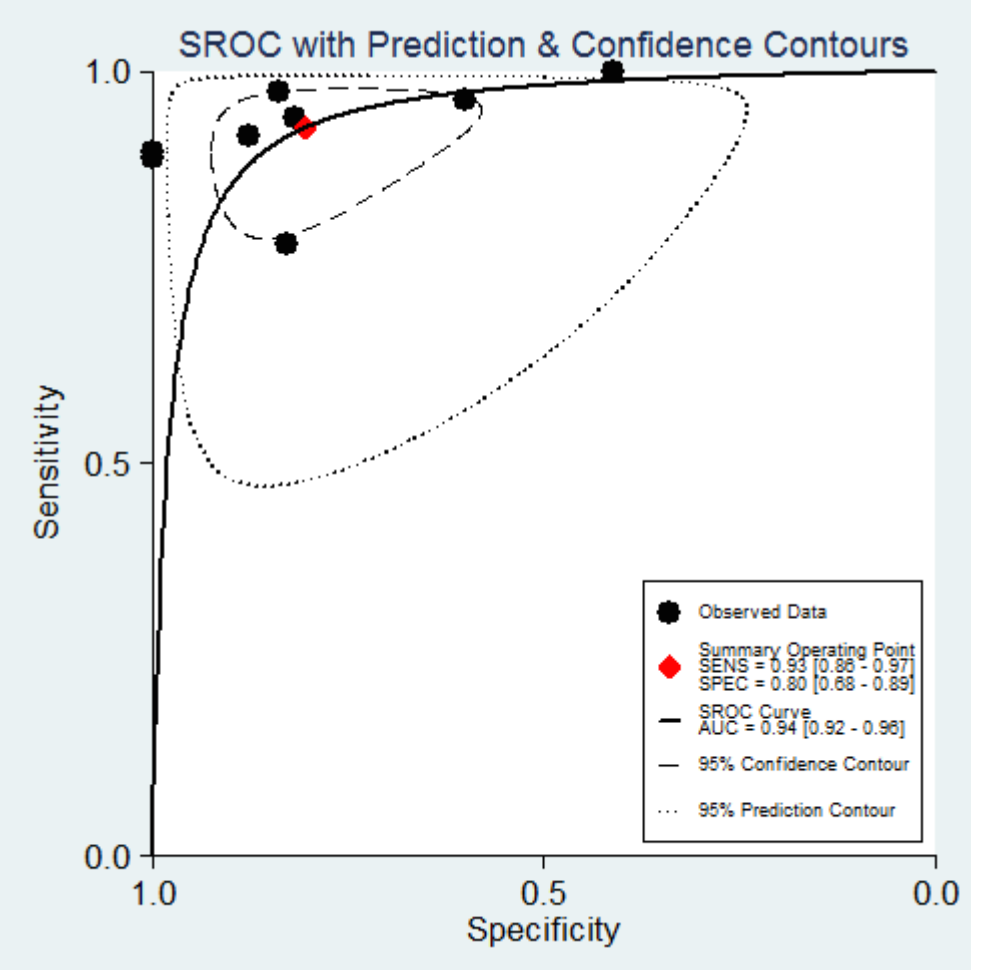


Our results confirmed data previously reported by Kraft et al. in their meta-analysis in 2011 (198). We performed an up-date of Kraft's results (198) and we obtained from their combination a final Se of $93 \%$ (95\% CI $0.86-0.97)$ and a value of $80 \%$ (95\% CI $0.68-0.89)$ for Sp, reporting a lower $I^{2}$ value for Se (67\%) than for Sp (77\%). (Figure 12).

Figure 12. Meta-analysis of sensitivity and specificity of AFE.

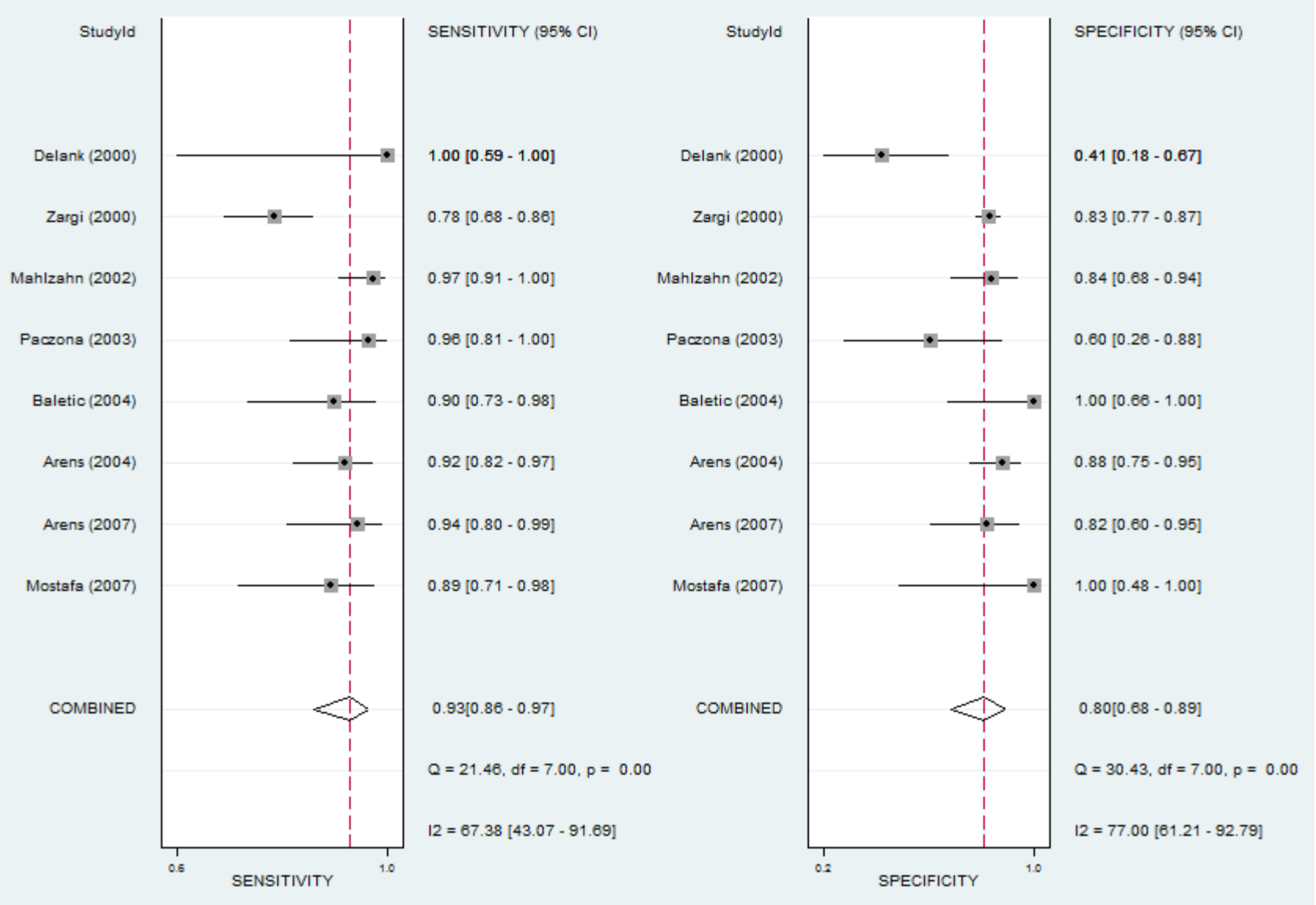

In specific, Succo et al. (201) highlighted evidence of advantages in allowing precise calibration of the entity of superficial resection during trans-oral laser surgery (TLS) as already reported by Lucioni and colleagues (202). Kraft et al. (198) through their review of literature found a lower J value for AF (0.75) in comparison to the latest publications, but they concluded that FAE is highly effective in the early diagnosis of laryngeal cancer and its precursor lesions, and that can be applied in the primary detection of these lesions providing a better evaluation of their horizontal extension and delineation. However, it is equally recognized that this tool is not as yet qualified to substitute for histological work-up. Baletic et al. (203) evidenced many conditions impacting AF features of laryngeal mucosa; while Rydell and colleagues (204) confirmed a high value of J index for AF (0.89). Saetti et al. (205) revealed a high sensitivity of the AF by the presence of demarcation of the cancer limits, with an increase in contrast between the normal and pathological mucosa. Here, the gap in sensitivity, between WL and AFE, resulted even more evident, about 37\%, than as reported in literature; in fact, comparing confident intervals, AFE revealed a significantly higher sensitivity 
than standard WL $(\mathrm{p}<0.05)$. Nevertheless, they insisted in specifying the need of a long learning curve and that risks are greatly operator-dependent.

The most recent diagnostic technique that has been introduced in the clinical practice is represented by the Narrow Band Imaging (NBI), in fact, first publications about this method are dated 2007. NBI has progressively showed very promising results of both $\mathrm{Se}$ and $\mathrm{Sp}$ for the differential diagnosis of laryngeal precancerous and cancerous lesions in the larynx thanks to its schematic classification of abnormalities of the intraepithelial papillary capillary loop (IPCL), that seems to have a direct matching with histological features, so far. In fact, Ni and colleagues (135) through their proposal of classification of ICPL, obtained a J index for NBI much higher than WLE (0.82 vs. $0.59)$, reaching statistical significance in terms of accuracy $(p=0.028)$, sensitivity $(p=0.020)$, and negative predictive value $(\mathrm{p}=0.048)$, in comparison to WL mode. Greater results, in terms of sensitivity, specificity and accuracy of NBI were obtained by Bertino et al. (138), who demonstrated the validity of this technique based on lesions' vascular pattern proposed by Ni and colleagues. Also Lukes and coll. confirmed high values of sensitivity and specificity for squamous cell carcinoma (100\% and $82 \%$, respectively) when IPCL were visible (130); and Kraft et al. (206) confirmed the capacity of NBI to easily detect and distinguish malignant and premalignant tumors from benign lesions, with a final accuracy of $97 \%$, with a $J$ value of 0.93 . Watanabe et al. (136) achieved in their cohort of patients a high sensitivity for the NBI, almost equal to AFE (91.3\%), but showed a higher specificity (91.6\%) compared with AF method. In addition, they demonstrated that NBI system can evaluate recurrent laryngeal cancers as well as newly developed superficial cancers, which are one of the drawbacks to using autofluorescence endoscopy (207). These results were confirmed by Zabrodsky et al. recently, in 2014 (208).

Irjala and coll. (132) supported same results about benefits of NBI accuracy by the addition of highdefinition television (HDTV). In this respect, Piazza et al. (137) showed that the use of high definition television (HDTV) NBI system, helped in increasing the rate of diagnosis significantly, from $20.8 \%$ (without HDTV) to $42.7 \%$, where the sensitivity rase up to $98 \%$ starting from $61 \%$, and specificity increased by $3 \%$, with a value of accuracy of $92 \%$ and a global $\mathrm{J}$ index of 0.88 , almost doubled in comparison to NBI alone. Thus, it seems that HDTV-NBI proves a significant accuracy in recognizing true positives and in distinguishing, at the same time, true negatives.

From our study we can affirm that values of Se and $\mathrm{Sp}$ of $91.44 \pm 13.279 \mathrm{SD}$ (95\% CI 81.941-100.939, median 97\%) and of $89.470 \pm 7.529$ SD (95\% CI 84.084-94.856, median 90.8\%), respectively; with a difference between $\mathrm{Se}$ and $\mathrm{Sp}$ not statically significant $(\mathrm{p}=0.5527)$. We constructed hierarchical SROC curve to assess the interaction between Se and Sp and these were $94 \%$ and $93 \%$, respectively with a AUC of 97\%. (Figure 13). 
Figure 13. SROC curve from bivariate model of NBI for cancerous lesions vs. precancerous lesions identification.

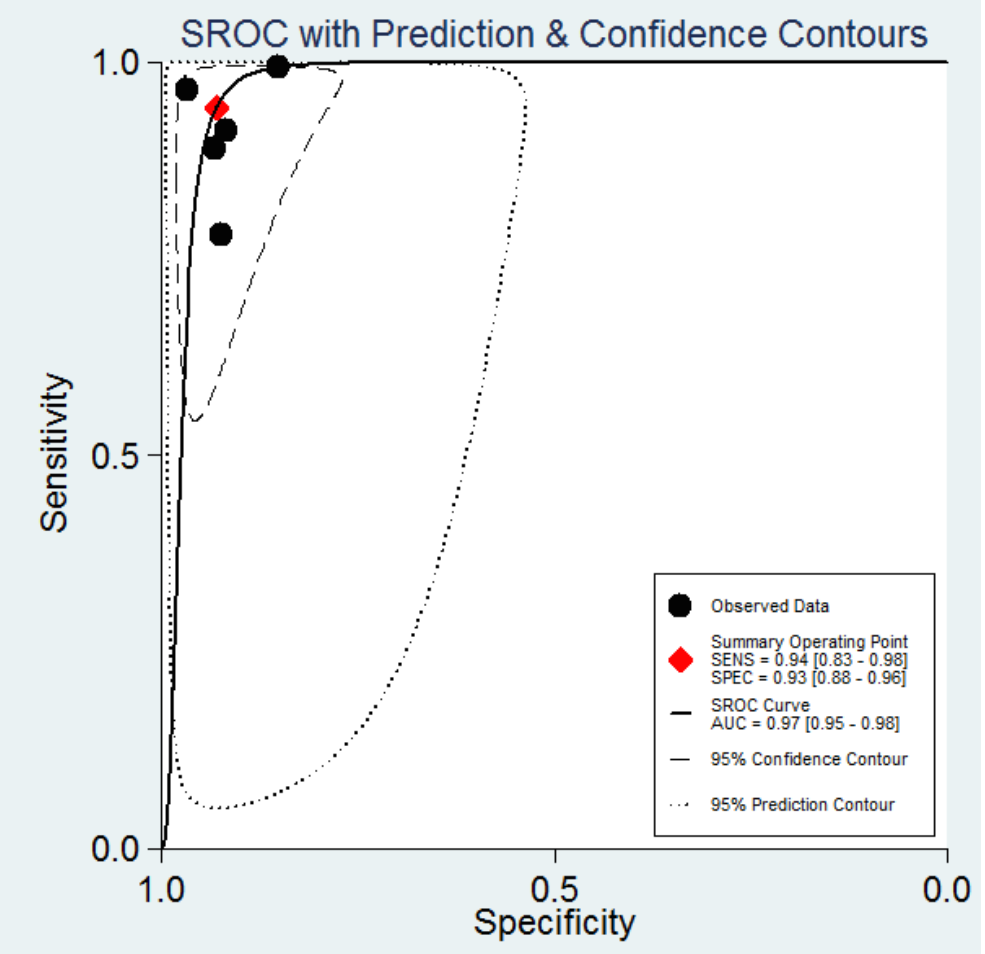

Then, we obtained from their combination a final 95\% CI $0.83-0.98$ for Se and a value of $95 \%$ CI 0.88-0.96 for $\mathrm{Sp}$, reporting a lower $I^{2}$ value for $\mathrm{Sp}(61.3 \%)$ than for $\mathrm{Sp}(87.48 \%)$. Here the hypothesis of hegemony among these different studies was about to be statistically significant $(\mathrm{p}=$ 0.04) (Figure 14). 
Figure 14. Meta-analysis of sensitivity and specificity of NBI.

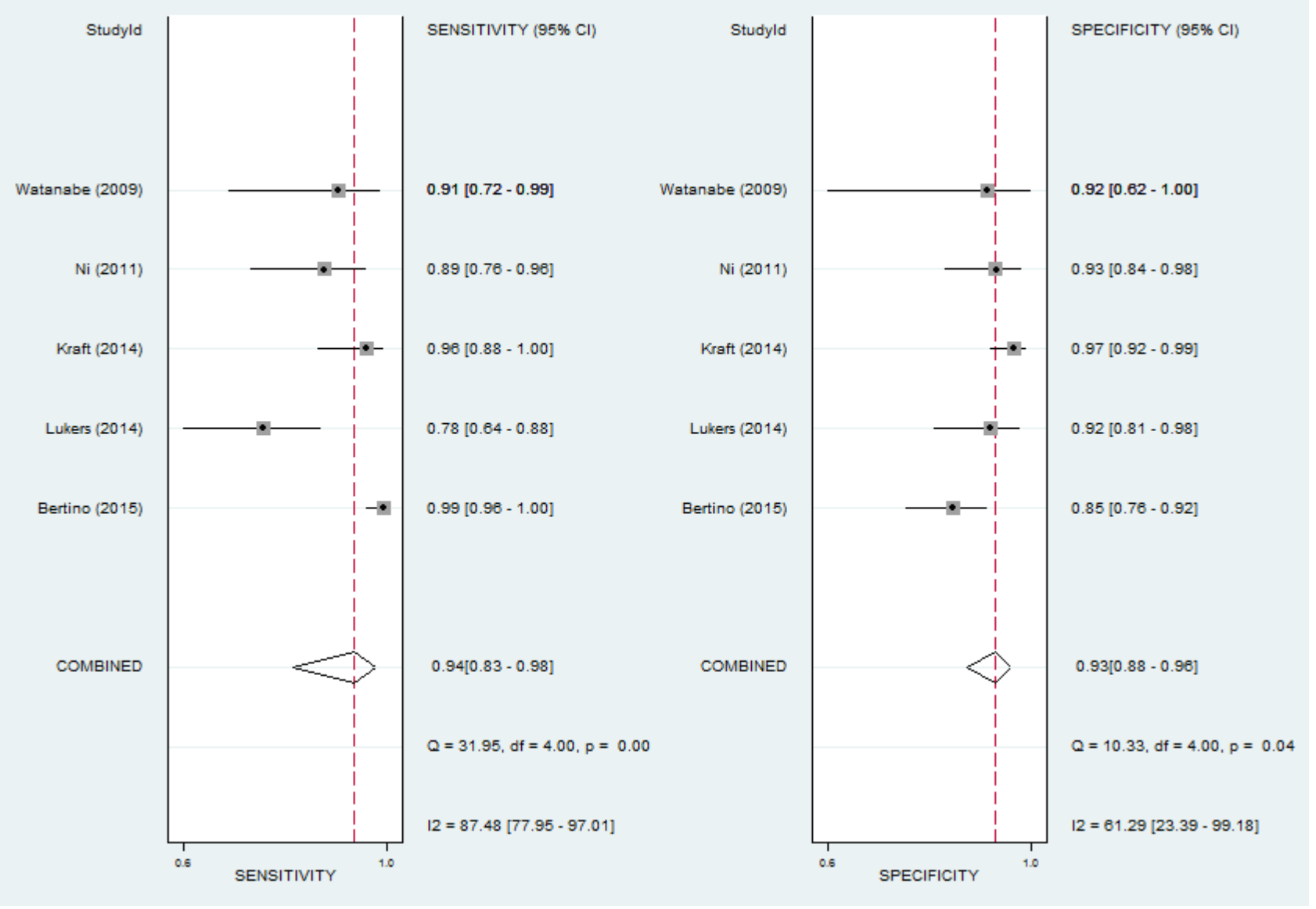

By comparing AFE and NBI values we can affirm that there is no statistical significancy in terms of both Se $(p=0.7268)$ and $\mathrm{Sp}(\mathrm{p}=0.1543)$, even if the AUC values revealed a slightly higher percentage for NBI (97\%) than for AFE $(94 \%)$.

If we make a comparison between AFE and WL, NBI and WL, we obtain interesting results. In fact, in terms of Se AFE showed a higher mean value of 91.914 \pm 3.383 SD than WL $(71.133 \pm 21.579$ SD) with a significant $\mathrm{p}$ value of 0.0229 ; while Sp did not show any statistical significant difference ( $p=0.8699$ ), with a mean value of $78.400 \pm 18.987 \mathrm{SD}$ for AFE and of $80.100 \pm 22.750 \mathrm{SD}$ for WL. If we make the same comparison between NBI and WL, there was a significant difference in terms of Se ( $\mathrm{p}=0.0283)$, referring to values of $88.422 \pm 7.171 \mathrm{SD}$ for NBI and of $71.133 \pm 21.579 \mathrm{SD}$ of WL; but, Sp did not show any statistical significance as well $(p=0.3056)$ where Sp of NBI $(89.470 \pm$ $7.529 \mathrm{SD})$ was higher than that one of WL $(80.100 \pm 22.750 \mathrm{SD})$.

- II group articles: advanced stage laryngeal tumor with cartilage involvement vs. early staged tumors assessment.

This group counted a higher number of good quality articles (13 vs. 5), where Se and Sp for determination of advanced laryngeal cancers with cartilage involvement presented a mean value of $78.268 \pm 20.611$ SD (95\% CI 69.760-86.776, median 84\%) and $82.140 \pm 17.335$ SD (95\% CI 
74.984-89.296, median 89\%), respectively, calculated on a global number of patients of 1136 with an amount of 497 diseased populations (people with cartilage involvement). Any statistical significance was found among continuos data $(\mathrm{p}=0.3855)$.

In the search for alternative imaging techniques, which could be performed by the treating physician, endosonography has become an important diagnostic tool from the 1960s'.

Preliminary results have reported a sensitivity of ultrasound in the assessment of laryngeal cancer of $84 \%$, with a specificity of $93 \%$ and an accuracy of $89 \%$; these values were statistical significant in comparison to CAT and MRI ( $\mathrm{p}<0.0001)$ in accordance with Kraft's results (209).

Recent investigations on human laryngeal endosonography, have demonstrated a penetrating depth of 10 to $25 \mathrm{~mm}$ (depending on the applying frequency) and a high tissue resolution. In fact, in 1994, Zech et al. (210) were first to report on this subject on cadavers; some years later, Arens et al. $(127,165,211)$ published an experimental study on sonographic anatomy of the larynx and the first clinical experiences with this promising method in a variety of laryngeal lesions through the use of a flexible miniprobes, with a frequency of $10-30 \mathrm{MHz}$ and a 360 field of view. Independently, Tamura et al. $(212,213)$ published similar clinical and experimental studies with the same probe. Tsui and colleagues (214) in 2011 first characterized the lamina propria and the vocal muscle by ultrasound in order to describe biochemical properties of the vocal folds.

Thus the promising properties of ultrasonography of the larynx, some authors made comparison among this emergent tool and standard diagnostic images acquisition. In fact, $\mathrm{Hu}$ et al. compared sonography to MRI (163) and CAT (160) in the evaluation and staging of glottic carcinoma, and their results revealed that there was no statistical difference among these two technique in pretherapeutic staging accuracy ( $80 \%$ vs. $76.7 \% ; \mathrm{p}>0.99)$, and $(83.3 \%$ vs. $88.8 \%$; $\mathrm{p}=0.735)$ respectively, suggesting ultrasonography as a noninvasive complementary modality for detection and initial staging of glottic carcinoma. In fact, when landmarks, i.e. ventricular bands and vocal cords, for localization are clearly identified by ultrasonography, laryngeal tumors could be correctly located ( $p=0.392$ ) (162). Moreover, they showed the utility of ultrasonography in diagnosing the neoplastic spread to the paraglottic space, evidencing its significative higher specificity in comparison to CAT (94.9\% vs. 66.7\%; p=0.001). With a final J value for sonography of 0.907 against a value of 0.667 for CAT.

From our few selected articles we can affirm that we obtained a Se and Sp for US of $88.067 \pm 3.828$ $\mathrm{SD}$ and of $91.367 \pm 5.244 \mathrm{SD}$, respectively, accordingly to recent reports.

About the predictability of CAT in the determination of laryngeal cartilage invasion it varies considerably among the most recent published reports (Table 13). With a low mean value for sensitivity of $64.194 \pm 20.862$ SD (95\% CI 53.468-74-921, median 66\%) in contrast to a higher mean value of $82.782 \pm 22.289$ SD for specificity (95\% CI 72.322-95.242, median 91\%). This comparison presents a significant $\mathrm{p}$ value of 0.0199 . 
Table 13. Sensitivity and specificity of CAT in identifying laryngeal cartilage invasion, various reports.

\begin{tabular}{|c|c|c|c|}
\hline Author & Year & Sensitivity (\%) & Specificity (\%) \\
\hline Castelijns et al. & 1988 & 46 & 91 \\
\hline Sulfaro et al. & 1989 & 47 & 88 \\
\hline Becker et al. & 1995 & 66 & 94 \\
\hline Zbaren et al. & 1996 & 67 & 87 \\
\hline Becker et al. & 1997 & 61 & 92 \\
\hline Amilibia et al. & 2001 & 54 & 91 \\
\hline Gordin et al. & 2006 & 88 & 8 \\
\hline Fernandes et al. & 2006 & 80 & 92 \\
\hline Jeong et al. & 2008 & 83.3 & 83.3 \\
\hline Beser et al. & 2009 & 88 & 50 \\
\hline Just et al. & 2010 & 87 & 95 \\
\hline Beitler et al. & 2010 & 49 & 92 \\
\hline Celebi et al. & 2012 & 89 & 89 \\
\hline Kraft et al. & 2013 & 68 & 84 \\
\hline Hartl et al. & 2013 & 11 & 94 \\
\hline Han et al. & 2013 & 57 & 94 \\
\hline Allegra et al. & 2014 & 50 & 100 \\
\hline
\end{tabular}

We can see how different reports are in contradiction; for instance, Gordin et al. (191) showed a CAT sensitivity in identifying cartilage involvement of $88 \%$, with a very low specificity ( $8 \%$ ); results that have been disproved by Allegra and colleagues (218) who found CAT values of 50\% (sensitivity) and 100\% (specificity), respectively for identification of cartilage invasion. Despite this conflicting final results, if we compare reports published before 2005 to the most recent works, we do not find any statistical significances among these two groups in terms of sensitivity $(\mathrm{p}=0.3367)$ and specificity $(\mathrm{p}=0.2574)$ for tumor cartilage involvement.

From our analyzed studies (see Table 12) we can affirm that values of Se and Sp of 71.750 23.428 SD (95\% CI58.223-85.277, median 81.25\%) and of 83.029 \pm 18.474 SD (95\% CI 72.362-93.695, median 91.8\%), respectively; with a difference between Se and Sp not statically significant $(\mathrm{p}=0.1691)$.

The pooled sensitivity and specificity of CAT for advanced tumors with cartilage involvement rate were $74 \%$ and $84 \%$, respectively, with a AUC of $85 \%$. (Figure 15 ). 
Figure 15. SROC curve from bivariate model of CAT for advanced stage cancers with cartilage involvement vs. early staged cancers identification.

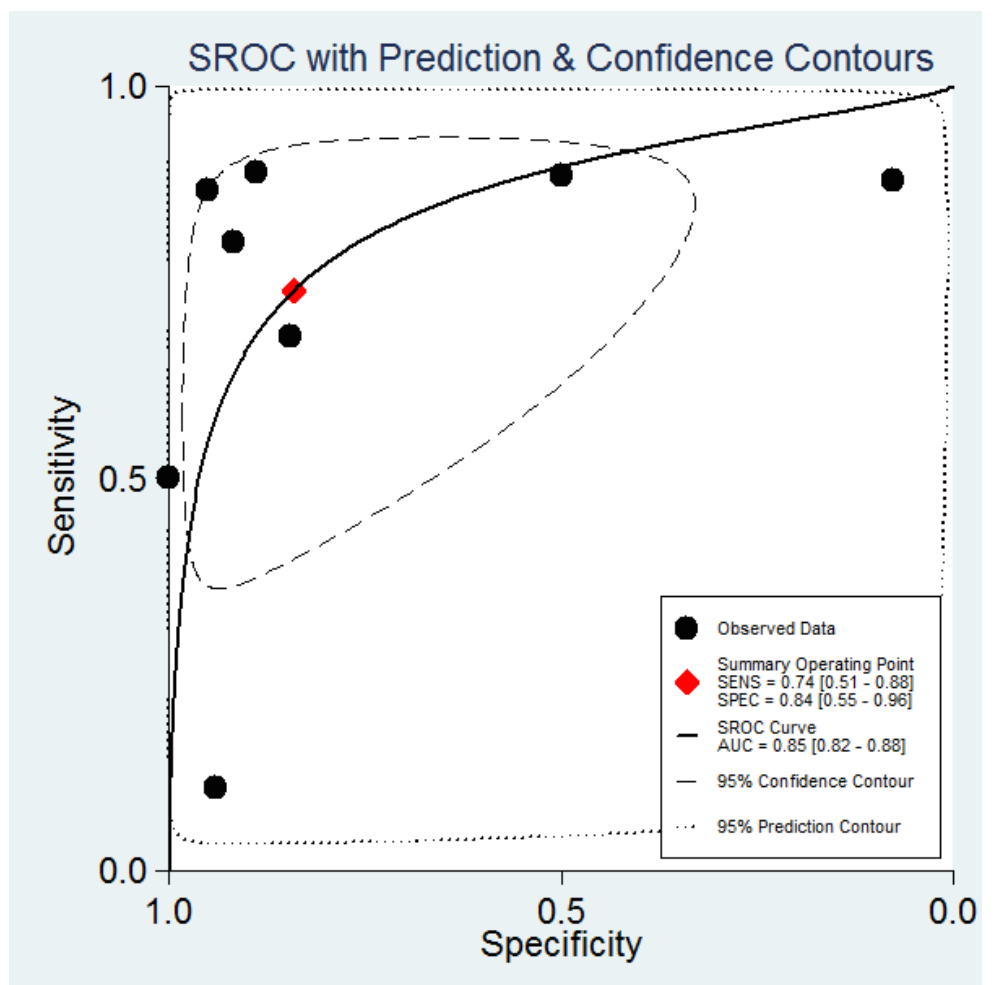

The Figure 16 provides the forest plot of studies that contributed to these results and the spread data with $95 \%$ CI for each study represented by horizontal lines. We obtained from their combination a final 95\% CI 0.51-0.88 for Se and a value of 95\% CI 0.55-0.96 for Sp, reporting a very high $I^{2}$ value for both Se (87.7\%) and Sp (95.91\%), where the lowest rates of Se were reported by Hartl et al. (220) and Allegra and colleagues (218), whilst Gordin and Beser $(191,222)$ showed the lowest rate for $\mathrm{Sp}$.

Here the hypothesis of hegemony among these different studies was not statistically significant (Figure 16). 
Figure 16. Meta-analysis of sensitivity and specificity of CAT.

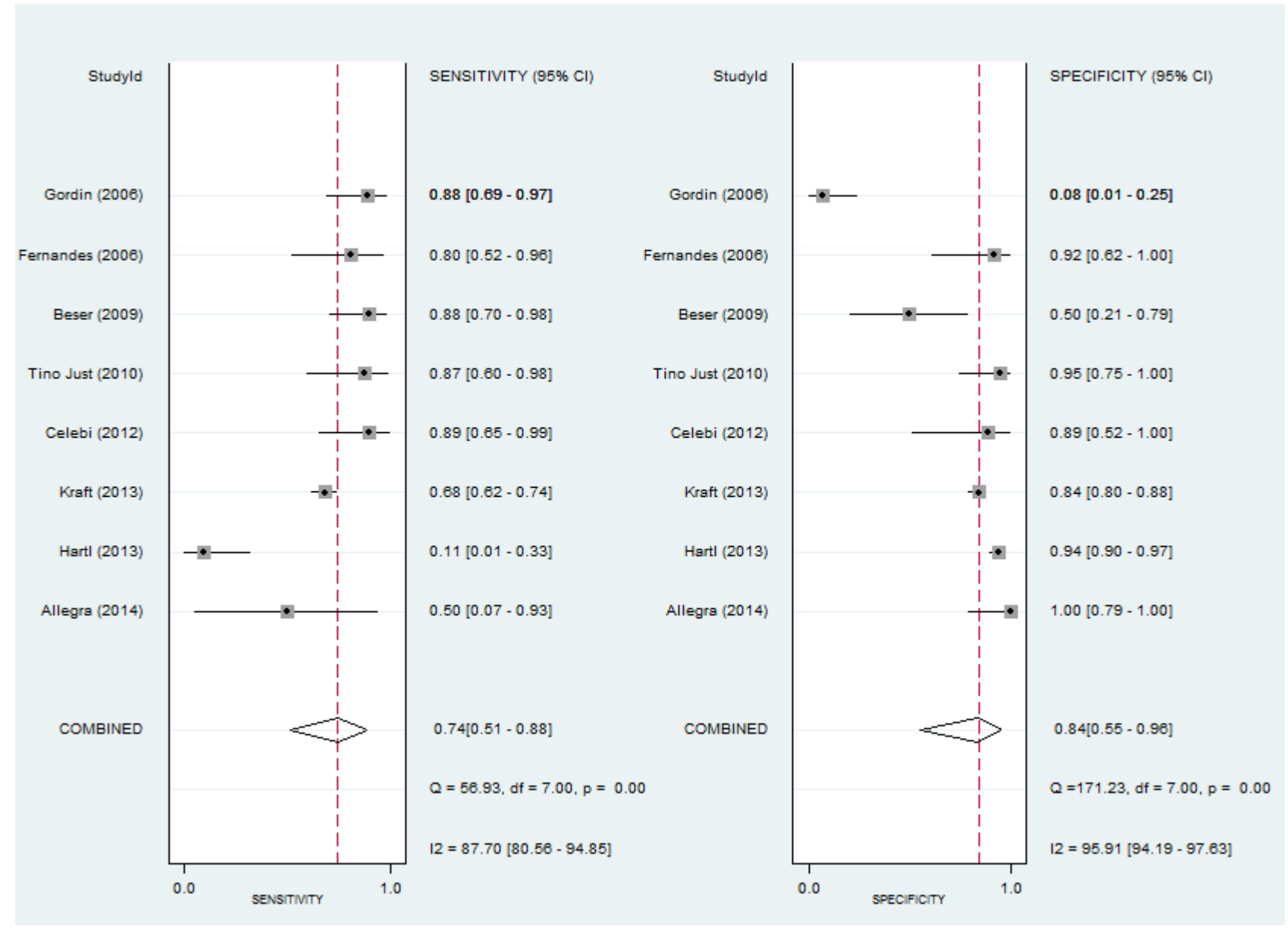

It is obvious that over the last decade the sensitivity of CAT has improved considerably, rising its median value by about $10 \%$ and thus canceling the previous reported significancy between Se and Sp of CAT in identifying laryngeal cartilage involvement.

From the 1980s' the magnetic resonance imaging (MRI) saw it enter in clinical practice.

Its predictability in the determination of laryngeal cartilage invasion varies considerably among published reports, as it has been shown of CAT as well (Table 14). With a mean value for sensitivity of $84.713 \pm 14.958$ SD (95\% CI 72.207-97.218, median $87.85 \%)$ in contrast to a mean value of $79.563 \pm 17.007$ SD for specificity (95\% CI 63.344-93.781, median 82.75\%) This comparison did not present a significant $\mathrm{p}$ value $(0.3321)$. 
Table 14. Sensitivity and specificity of MRI in identifying thyroid cartilage invasions, various reports.

\begin{tabular}{|c|c|c|c|}
\hline Author & Year & Sensitivity (\%) & Specificity (\%) \\
\hline Becker et al. & 2008 & 96 & 75 \\
\hline Lim et al. & 2011 & 84.8 & 89 \\
\hline Banko et al. & 2011 & 100 & 93 \\
\hline Kinshuck et al. & 2012 & 64 & 67 \\
\hline Kraft et al. & 2013 & 63 & 89 \\
\hline Shang et al. & 2013 & 90.9 & 76.5 \\
\hline Banko et al. & 2014 & 79 & 47 \\
\hline Allegra et al. & 2014 & 100 & 100 \\
\hline
\end{tabular}

Those reports that were analyzed for the meta-analysis showed through the SROC curve an interaction between Se and Sp of $86 \%$ and $83 \%$, respectively with a AUC of $91 \%$. (Figure 17).

Figure 17. SROC curve from bivariate model of MRI for advanced stage cancers with cartilage involvement vs. early staged cancers identification.

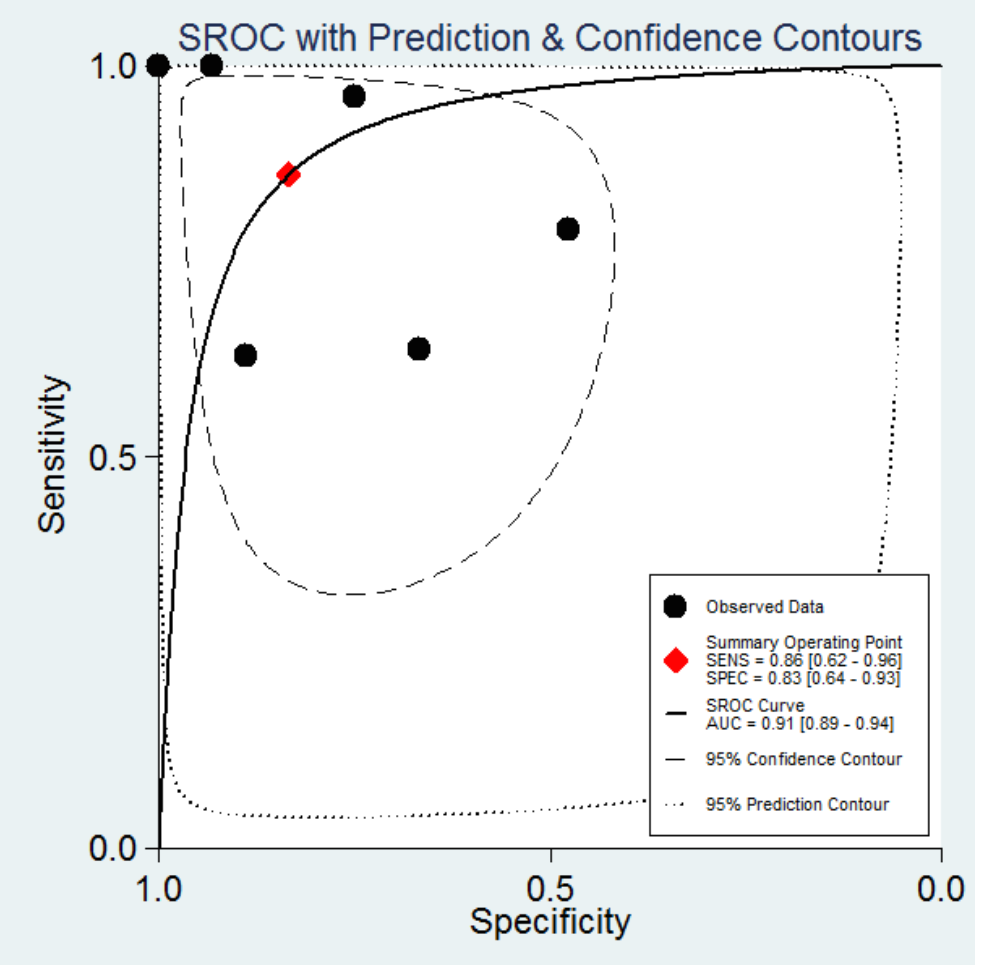

The Figure 18 provides the forest plot of studies that contributed to these results and the spread data with $95 \%$ CI for each study represented by horizontal lines. The pooled sensitivity and specificity of MRI for advanced tumors with cartilage involvement rate were $86 \%$ and $83 \%$, respectively, with a 
final $95 \%$ CI $0.62-0.96$ for Se and a value of 95\% CI 0.64-0.93 for Sp. Moreover, heterogeneity of the studies included in this analysis was very high for both sensitivity (83.29\%) and specificity (83.79\%), where the lowest rates of Se were reported by Kraft et al. (209), whilst Banko et al. (224) showed the lowest rate for Sp. Here the hypothesis of hegemony among these different studies was not statistically significant, too.

Figure 18. Meta-analysis of sensitivity and specificity of MRI.

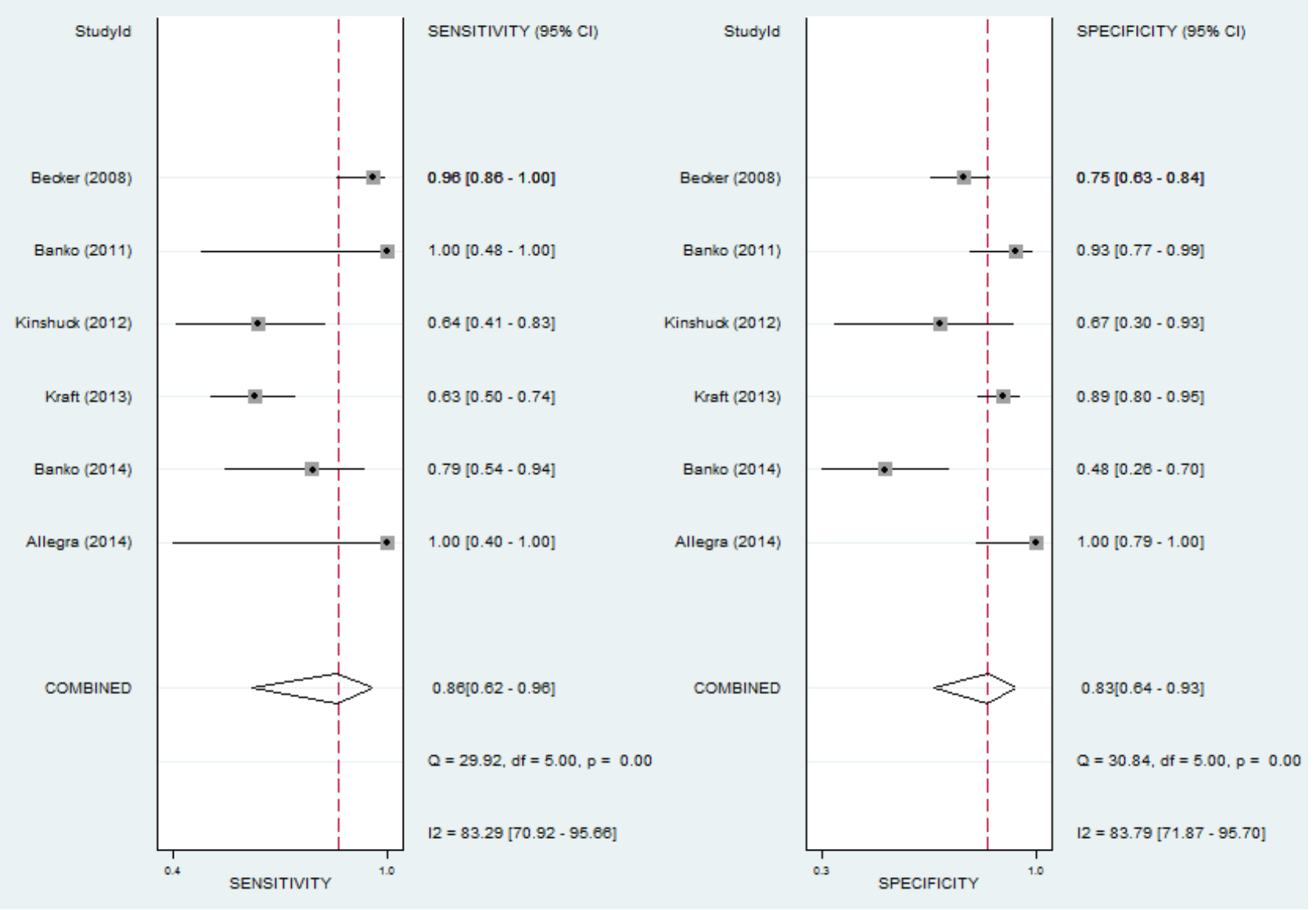

In the 1990s' PET and CAT/PET entered into the clinical practice and Gordin et al. (191) showed how the combination of CAT and PET can reach a better sensitivity and specificity in comparison to PET study by itself ( $92 \%$ and $96 \%$ vs. $92 \%$ and $73 \%$, respectively). But, for the first time, Jeong et al. (193) showed that PET/CT findings do not add to the conventional work-up for the initial evaluation of glottic cancer any further advantages that would support the additional cost of this exam.

By comparing CAT and MRI values we can affirm that there is no statistical significancy in terms of both $\mathrm{Se}(\mathrm{p}=0.1708)$ and $\mathrm{Sp}(\mathrm{p}=0.6274)$, even if the AUC values revealed a higher percentage for MRI (91\%) than for CAT (85\%). 


\section{Discussion.}

Advanced image analysis procedures are used in laryngeal diagnostics with increasing frequency. Nonetheless, the image analysis procedures used are quite often limited to image visualization, for example, in indirect autofluorescence laryngoscopy. Quantification of color, texture, and shape of lesions and normal tissue could help in a more accurate categorization of lesions as well as in follow-up procedures.

\section{- White light endoscopy and stroboscopy.}

WLE together with stroboscopy remain the clinical key element for detecting and assessing vocal fold lesions, representing the essential diagnostic technique for evaluation of laryngeal mucosa and vocal fold motion biomechanics. Videostroboscopy especially, is the most practical and useful procedure for clinical assessment of viscoelastic properties of the phonatory mucosa, seems to be the reason for the superior sensitivity and specificity of WLS in glottic lesions over other diagnostic methods with continuous non stroboscopic light sources such as AFE. It realizes beneficial visualization and quantification of mucosal wave properties and phonatory oscillations, allowing reliable conclusions in terms of clinical diagnosis.

In this setting the article written by Cohen and colleagues (230) is worthy to be mentioned because, even if it did not meet all of the inclusion criteria to be enrolled for our meta-analysis, it showed a high quality score of 7 and gave the image of the actual skyline about general ear-nose-throat (ENT) practice in the U.S.A. analyzing factors influencing the common clinical practice and how this could engrave patients management.

It is well known that stroboscope is critical in the diagnosis of voice disorders, it can alter the treatment decision in $14 \%$ to $33 \%$ of cases in otolaryngology practice (86). Djukic et al. (231) evidenced that the presence of some factors at the stroboscopic analysis placed patients at higher risk of recurrence and progression of the disease.

In clinical practice, decision-making is quite often based on subjective evaluation of video data, but stroboscopy will continue to be a useful tool for the clinical evaluation of the patient with dysphonia as long as the clinician is aware of the deficiencies of stroboscopy (99). In terms of quantitative evaluation of the VLS using basic parameters this tool seems to be reliable in clinical settings with high sensitivity and specificity distinguishing healthy and pathological voice patients groups, with a low interclass correlation (ICC) value, ranging from 0.2 to 0.9 (232). Limitations of quantification of VLS variables originate from inherent restrictions of stroboscopic examination, which is basically an optical illusion made by the human eye, arising from the virtual reconstruction of adjacent phases of periodic oscillatory samples (200).

Accordingly to these limits, a combination of VLS with other diagnostic procedures is advocated. Peretti et al. (233) in their study showed $82 \%$ specificity and $100 \%$ sensitivity of VLS in diagnostic of glottal carcinoma; however, the combination of VLS with saline infusion into Reinke space raised the values of specificity up to $89 \%$ and sensitivity up to $100 \%$. Gugatschka et al. (91) demonstrated that the combination of cytology and pathological VLS allowed detection of glottic cancer with a sensitivity of $97 \%$, in contrast to $74 \%$ as found by cytology alone. 


\section{- Contact endoscopy.}

CE allows for the detection of very early pathologic areas not visible with WL or not properly interpreted with image-enhanced endoscopy (IEE) alone by targeting biopsies, which reduces the chance of under treatment. Contact endoscopy has, in the hands of an experienced examiner, a high negative predictive value (97\%); nevertheless, it must be measured against the current gold standard, the histopathological assessment of the biopsy samples.

This technique has shown a number of advantages: (1) ensures a non invasive and safe repeatable method; (2) when performed pre-operatively it provides an insight into the microscopic margins of the laryngeal mucosa lesion; (3) it can be used intra-operatively to ensure that the lesion has been completely removed; (4) it allows visualization of the microvascular net, which can assist the initial diagnosis of early laryngeal lesions. As CE supplies intraoperative information on the type of laryngeal pathology, thus significantly influencing the subsequent operative course, the criteria for malignancy are set high in order to minimize the use of unnecessary operative procedures as already demonstrated by Puxeddu et al. (102), and by Ledda et al. (111), confirming the significant role of ECE for more comprehensive staging and management of early cancer of the UADT. In fact, false negative result will only delay optimal operative or other therapeutic intervention until histopathology results are obtained, whereas an operative procedure performed on the basis of a false positive result may imply major and irreversible error. In this view, CE can be very useful to the surgeon, and the operative procedure can be more safely continued on the basis of the findings obtained.

Warnecke et al. (106), calculated the inter-rater reliability (k value) which was of 0.81 between the examiners rating contact endoscopic findings, thus showing an obvious correlation between the diagnosis of the experienced and inexperienced examiner. However, when compared to the current state-of-the-art diagnostic measure, histopathology, a stronger correlation with the otolaryngologist (kappa 0.81) than with the cytopathologist (kappa 0.61) was found. It is striking that the otorhinolaryngologist tended to overrate malignancies.

On the base of Cikojevic results (107) obtained from contact endoscopy are therefore nearly as reliable as frozen section pathology. Intraoperative contact endoscopy shall be considered as an addition to histopathology rather than as a replacement. Accordingly, the advantages of contact endoscopy are the immediate results, its instant availability, and the possibility of examining many mucosal areas in a short time and directly during surgery.

\section{- Autofluorescence.}

When imaged with an autofluorescence system, healthy laryngeal mucosa fluoresces bright green, whereas neoplastic mucosa appears red-violet (234), probably as a result of the thickened neoplastic epithelium preventing the illuminating light from reaching the basement membrane and collagen, which would normally provide fluorescence. In 2006, Arens and his colleagues (130) affirmed that it is not possible to detect the responsible endogenous fluorophore from the remitted autofluorescence light because it is a summation spectrum, and in thickened precancerous and cancerous mucosa $(>300 \mu \mathrm{m})$ the light beam is not able to penetrate. On the contrary, Wang et al. (235) described a penetration depth of blue light $(400 \mathrm{~nm})$ in mucosal tissue of $500 \mu \mathrm{m}$, whilst 
Gottschalk (236) found a penetration depth of just $300 \mu \mathrm{m}$. Therefore, normal vocal fold mucosa with a thickness of $120 \mu \mathrm{m}$ can easily be penetrated by the light beam.

Several studies have demonstrated that autofluorescence endoscopy and spectroscopy are better at identifying invasive carcinoma when compared with microlaryngoscopy alone $(116,119,204,128,129)$; but Dobre et al. (237), admitted to have problems in differentiating different grades of dysplasia, and to not be able to distinguish in situ carcinoma from invasive cancer.

In summary, despite the reported higher sensitivity value (93\%) of AFE, which compared to the white light endoscopy Se has improved in percentage showing a statistical significancy of $p=0.0229$, together with a better AUC value (94\% for AFE vs. 91\% for WLE), these mixed results of literature $(116,126,128,130,238)$ image the high grade of heterogeneity found in our study, that could be linked the high incidence of false positive results with a still rather low $\mathrm{Sp}(80 \%)$.

In fact, due to the high absorption of excitation light by haemoglobin, false positives (decreased autofluorescence with a nonmalignant histopathology) occurred more frequently in the presence of highly vascularized lesions such as telangiectasic polyps, granulation tissue, papillomas; while they were relatively common in case of scar tissue or pronounced local inflammatory reactions of the mucosa. Bacterial plaques and necrotic tissue can lead to a defect of autofluorescence that leads to false positive findings.

On the other hand, false-negative findings [negative autofluorescence despite (pre)malignant histopathology] were generally rare and resulted mostly from pronounced hyperkeratosis of the examined areas that caused optical "masking" of clinically significant lesions. A dark field in an image may be the results of a shadow from an anatomic structure over this field. In attention to avoid false positive and false negative findings, we recommended simultaneous careful comparison of white light and autofluorescent images of same view.

Both effects: (1) epithelial thickening of (pre)malignant tissue with inhibited penetration of exciting light into submucous layers and (2) the altered metabolism of tumor cells with lower concentration of NADH and FAD, are claimed to be mainly responsible for the reduced total AF in neoplastic laryngeal tissue (197).

These considerations are basic as far as concerns the application field of this method. In accordance with Delank et al. (128), we consider that AFE is not useful in the evaluation of "frankly benign" laryngeal lesions.

\section{- Narrow Band Imaging.}

The introduction of narrow band imaging (NBI, Olympus Medical System Corporation, Tokyo, Japan), already proven to be an useful screening method in other medical fields, has recently shown its potential in identifying carcinomas at an early stage in head and neck mucosal sites $(136,139,239,240)$. The NBI filter sets $(415 \mathrm{~nm}$, which is the hemoglobin absorption band, and 540 $\mathrm{nm}$ ) are selected to obtain fine images of microvascular structures of different types tissue, which are necessary for diagnosing a tumor at early stage.

$\mathrm{Ni}$ and colleagues (135) proposed a classification based on the superficial vascular patterns in laryngeal lesions enhanced with NBI, with a diagnostic accuracy of $90.4 \%$ (sensibility, specificity, and positive predictive value were respectively $88.9 \%, 93.2 \%$, and $90.9 \%$ ) in detecting malignant 
lesions from carcinoma in situ to invasive carcinoma; they correlated the superficial spots to vascular anomalies, but the technique with only NBI offers only a partial and sometimes subjective interpretation of the patterns, not allowing to recognize the deepest changes of the vasculature in the chorion. Previous studies have not described intraepithelial papillary capillary loop features in detail, and their data analysis indicated that intraepithelial papillary capillary loop features were closely related to histopathological findings with a statistical significance. Thus, the classification of intraepithelial papillary capillary loop features may facilitate the prediction of laryngeal cancer or precancerous lesions. However, the presence of necrotic tissue or a thick white patch on lesions may affect the evaluation of intraepithelial papillary capillary loop features, especially for invasive carcinoma, resulting in false negative findings.

From our results NBI showed a rise in Se in comparison to AFE (94\% vs. 93\%), together with an improvement of its $\mathrm{Sp}$ which increased of more than 10\%, but without any statistical significancy ( $\mathrm{p}=0.1543$ ). This could be explain by the high grade of $I^{2}$ for $\mathrm{Sp}(87.48 \%$ for NBI vs. $77 \%$ for AFE) which seems to be correlated to the unavoidable subjective interpretation of optical features requested by these two tools. If we compare Se of NBI and WL, a further significant result comes out $(\mathrm{p}=0.0283)$, highlighting the evident and promising improvement in sensitivity and falsenegatives incidence guaranteed by these two diagnostic techniques whose preliminary results have been first published in 1995 and 2007, respectively. Moreover, NBI showed a further increase in accuracy in comparison to AFE, reaching a value of AUC of $97 \%$.

In specific, AFE in comparison with NBI, presents a lower specificity because some benign lesions also display a loss of green fluorescence; in addition, mucosal scars and inflammation often cause false positive results (Johnson FL. Management of advanced premalignant laryngeal lesions. Curr Opin Otolaryngol Head Neck Surg 2003;11: 462-6;); in contrast, Ni et al. (135) indicated that these conditions, classified as type II or III under NBI, can be easily differentiated from type V lesions. Lukes et al. declared that their result may be influenced by very low numbers of benign lesions in their study group (150), and that some of the associated endoscopic characteristics to each single laryngeal lesions, such as the surface, the presence of multiple lesions, and the spread to both vocal cords, may be taken as the criteria used for a more accurate diagnosis.

\section{- Ultrasound.}

In the literature, there exist only a few publications on endosonography of the larynx. Most of them were purely experimental studies on cadaver specimens (210,211,213). In 1999, Arens et al. (165) published the first clinical experiences in 38 patients presenting with 23 laryngeal cancers and 15 benign lesions. Two years later, Tamura et al. (212) performed a retrospective study of 16 patients with 2 malignant and 14 benign vocal fold lesions. Both authors used $360^{\circ}$ radial scanning flexible miniprobes with a frequency of 10 to $30 \mathrm{MHz}$. Because the interpretation of endolaryngeal sonograms can be influenced strongly when the definitive histopathology is already known, the predictive value of endosonography can be depicted only if an investigation is performed in a prospective and blinded manner. To our knowledge, the study of Kraft et al. (209) is the only prospective clinical trial assessing the value of endosonography compared with CT and MRI in 
staging laryngeal cancer. For this reason, a real comparison of our results with the literature is not possible. Generally, there are no contraindications to perform endosonography of the larynx.

The two main limitations of laryngeal ultrasonography are the thyroid cartilage ossification and the air contained in the larynx (163). But modern real-time high-frequency sonography has remarkably improved the imaging resolution (241). Despite these limits, one study showed for sonography a sensitivity in detecting laryngeal cartilage invasion of $100 \%$ (242), which is a well known crucial concern for patients outcome. Rothberg et al. (243) stated that sonography was superior to unenhanced computed tomography for evaluation of cartilage invasion. Microscopic invasion of the cartilage may be missed at the ultrasonography. Several authors $(159,160,163)$ reported an accuracy rate for detection of anterior commissure involvement of $86.7 \%$ for ultrasonography, $80 \%$ for MRI and $89 \%$ for CAT, without any statistical significance $(\mathrm{p}=0.688)$; together with accuracy values on sonography of $83.3 \%, 80 \%$, and $83.3 \%$ for supraglottic, subglottic and paraglottic areas respectively, on MRI of $90 \%, 83.3 \%$, and $86.7 \%$, and on CAT of $94.4 \%, 88.9 \%, 91.7 \%$ (p>0.99).

Our results confirmed these promising prospectives showing a global Se value of $88 \%$ with a higher percentage of $\mathrm{Sp}(91 \%)$.

\section{- Computed Axial Tomography and Magnetic Resonance Imaging.}

Both CAT and MR imaging are routinely used for detection of subtle cartilage invasion, but there is still controversy about which modality can most accurately detect cartilage invasion, and both modalities have shortcomings (175).

CAT continues to be widely used for diagnosing cartilage invasion. In fact, although MRI is significantly more sensitive, it is less specific than CAT in detecting neoplasm cartilage invasion $(177,185)$. Advantages of MRI include superior soft tissue contrast and exact delineation of tumor margins $(220,244)$, where the cartilage invasions is usually overestimated for anterior commissure tumor.

Radiologic criteria for CAT diagnosis of cartilage invasion include sclerosis (increased density of the cartilage or high attenuation), lysis, erosion, and extra-laryngeal tumor spread (cartilage discontinuity with tumor extending through both the inner and outer cortices). When determining whether erosion or lysis is present or absent, differentiation from cartilage invasion may sometimes be difficult using conventional CT; some cases may be distinguished from erosion if two concurrent findings of negativity are identified: a perfect or almost continuously defined thin hypo-attenuating line between the tumor and the cartilage, and CT attenuation of non-ossifying cartilage that differs from that of the tumor. Demonstrating tumor invasion of non-ossified cartilage is problematic with CT due to similarity of the CT density (100 HU), making them almost indistinguishable, especially when the tumor is located adjacent to non-ossified cartilage. In addition, the appearance of laryngeal cartilage on $\mathrm{CT}$ varies widely according to differences in the proportions of hyaline cartilage (which ossifies with aging), cortical bone, and fatty marrow, which complicates interpretation.

On the other hand, MRI is more sensitive (sensitivity up to 96\%) in this situation since tumor is of increased T2-w signal relative to non-ossified cartilage (175). Unfortunately MRI has a tendency to overestimate cartilage involvement by tumor since it may be indistinguishable from that due to peri- 
tumoral inflammation. These changes are most commonly seen in the thyroid cartilage so specificity is lowest at this location. New criteria of moderately high T2-w signal and moderate enhancement as compared to the marked signal changes and gadolinium enhancement with inflammation, have been proposed to overcome this problem. Alternatively the lower specificity of MRI may be addressed by performing a corroborating CT scan in the presence of an MRI scan positive for cartilage invasion (245). The introduction of multi-slice CT has resulted in an increase of spatial and temporal resolution but has led to little progress in interpretation of cartilage invasion which is still sometimes overestimated. If the cartilage displays a signal intensity similar to that of the tumor, cartilage invasion should be suspected. Contrast-enhanced MR imaging is also useful when evaluations by $\mathrm{CT}$ alone are insufficient for excluding cartilage invasion, and have reached high sensitivity (96\%) and high negative predictive values of up to $96 \%$, but still maintaining a low specificity $(178,229)$. However, the MR findings suggestive of cartilage invasion are not specific, and therefore may lead to a number of false positive signs. The reason is that reactive inflammation, edema and fibrosis in the vicinity of a tumor may demonstrate diagnostic features similar to those of cartilage invasion. Inflammatory changes are most common in the thyroid cartilage, and therefore the specificity of MR imaging for detecting invasion of the thyroid cartilage is only 56-65\% (229). Furthermore, due to motion artifacts that degrade images resolution, together with lacks of thin sections and the easier incidence of overestimation of tumor extension, MRI is not a satisfactory first choice for imaging of laryngeal and hypopharyngeal cancer (175). Despite these differences in $\mathrm{Se}$ and $\mathrm{Sp}$, there is no statistical significance between CAT and MRI in cartilage invasion assessment (175).

In literature, the pretherapeutic staging accuracy of laryngeal carcinoma has been reported to be 86 and $87.5 \%$ with CAT and MRI, respectively (246); moreover, MRI is more sensitive than CAT and presents $\mathrm{h}$ a high negative predictive value for detection of neoplastic cartilage invasion $(177,215)$. On the other hand, CAT is more specific, but less sensitive method than MRI for the detection of neoplastic cartilage invasion (229). In this respect, cartilage invasion is critical not only for tumor staging, but also for outcomes (229).

Our results despite the higher accuracy value of MRI (91\%) than of CAT (85\%), no statistical significances were issued. This could be explain that further more accurate analysis on specific subgroup of patients with thyroid cartilage involvement should be carried on for both tools by using similar techniques and coils, especially for MRI.

\section{Limitations.}

All papers included in this study are retrospective works mainly from individual institutions and are prone to the bias involved with retrospective studies. While objections can be raised about the pooling of different diagnostic procedures under the same group and the high level of heterogeneity in the meta-analyses, the inclusion of over 4400 patients makes the results fairly robust. This study was unable to access non-English language papers owing to limited resources. The authors suspect that addition of this literature will not substantially alter the conclusions of this study, given the large numbers that have contributed to the results. 
In addition a substantial cohort of patients in non-English language literature would have been duplicated in English language literature as these procedures are done by select centers who have published extensively in both English and non-English language.

\section{Conclusions.}

- Limitations of laryngoscopy and clinical examination include: submucosal intralaryngeal growth of tumors and invasion of the laryngeal cartilages and of the extralaryngeal soft tissues cannot be detected and large supraglottic and glottic tumors create difficulties in evaluating portions of the larynx more caudal in location.

- The discrepancy in diagnosis between video-laryngo-stroboscopy (VLS) and suspension microlaryngoscopy (SML) highlights certain key points: (1) additional glottic lesions could be noted in less than $10 \%$ of cases; (2) SML does not alter diagnosis but might lead to undertake management changes; (3) accordingly, surgical consent must be exhaustive and specifically address the notion of "hidden" lesions, some of which may influence the extent of surgery performed. The patient age, treatment modality, and stroboscopic signs, such as abnormal amplitude of vocal fold vibration and the existence of non vibrating segment, can be considered as warning factors for recurrence and disease progression and discussion of possible contralateral vocal fold involvement is recommended.

- In our opinion, the clinical experience of the examiner regarding precise application and evaluation of the available WLS appears to be more reliable in diagnosing vocal fold lesions than AFE, which is a relatively nonspecific method for diagnostics of mucosal tissue changes. AFE can be proposed as a promising noninvasive optical diagnostic procedure in more than $85 \%$ of cases, but the interpretation of its results requires knowledge of its basis principles and pitfalls of this technique.

- Contact endoscopy offers a cost-saving, fast, repeatable, and risk-free examination that could considerably reduce the time to diagnosis or at least help to localize the region of interest to enable more specific biopsy sampling for histological evaluation. But, thus it gives information on only the three most superficial cell layers, due to the limitation in staining with methylene blue, further investigations are necessary to develop dyes that can also stain deeper cell layers.

- The AFE procedure provides a better evaluation of the horizontal extension in cancerous lesions then WLE alone. It is short, easy to perform and without complication and biopsy is not required; thus, it could significantly improve the diagnostic efficacy of laryngeal pathology, due to a more precise assessment of boundaries of lesion with AFE, and the fact that AFE could serve as a guide for taking biopsy of lesion and endoscopic laser resection of laryngeal lesions. On the other hand, an intrinsic limit of the method is the impossibility to evaluate the extension of the carcinoma in the deeper tissues and, therefore, to differentiate between carcinoma in situ and invasive carcinoma.

- NBI endoscopy can be performed during a normal daily work in outpatient clinic by pushing a fingertip control switch, without using special techniques, without drug application. The most prominent feature of this technology both in the pre- and intraoperative setting is a more accurate 
definition of the neoplastic superficial spreading with consequent improvement of the peripheral margins control and modulation of the custom tailored endoscopic treatment. It is, however, important to understand that it is not black and white reading and therefore one still needs an experienced clinician on the field and, even with an expert, a learning curve of the new technique has to be taken into account. During this period one has to be prepared for more time-consuming examination and very likely for some extra biopsies before the eye is trained for the NBI.

- US present irrefutable advantages in comparison to CAT and MRI scans, which count: a lower benefit-costs ratio, absence of swallowing artifacts due to the general anesthesia, a real-time and repeatable mode, a closer relationship between diagnostics and therapy thus the treating surgeon performs the diagnostic technique at the same time. Then, it is suggested as a noninvasive complementary modality for the detection and initial staging of glottic carcinoma. On the other hand, disadvantages such as its limited availability, the need of performing this procedure under general anesthesia, lesions smaller than $3 \mathrm{~mm}$ are not detectable (anyway, still earlier than with CAT or MRI), together with its strong dependence on the examiner's experience, make the use of this tool optional and not straightforward.

- Imaging, conventionally with computed axial tomography (CAT) and/or magnetic resonance imaging (MRI), is nearly always required as endoscopic staging alone may underestimate the extent of the tumor. Both can enable the accurate prediction of the site, size, and spread patterns of laryngeal tumors. MRI is more indicated for unilateral lesions due to its higher Se $(96 \%)$ and NPV (96\%) than CAT in identifying cartilage involvement, even if MRI presents a higher incidence of false-positives. In these cases a CAT scan could be performed in order to increase the specificity of the study. In case of bilateral vocal cords lesions, CAT seems to improve its Se and it could be proposed as first line diagnostic pre-treatment tool.

We could summarize this bullet list in the following flow-chart (Figure 19).

Figure 19. This flow-chart images the diagnostic steps that would be suggested for the clinical assessment and management of laryngeal lesions suspicious for precancerosis or cancer. Two different types of scenario are reported: 1 . Unilateral vocal cord lesion, and 1. Bilateral vocal cord lesion/s. Once white light endoscopy plus stroboscopic study represent standard office-based analysis, further following steps depends on the previous analysis result. The presence or absence of the mucosal wave at the stroboscopic test influences the management of the patient. In the first case an intraoperative phase is planned, on the other hand, the second scenario worths second level diagnostic tools, such as MRI and/or CAT. Then, an intraoperative superficial and/or threedimensional visualization of the lesions is mandatory. One tool among CE, AFE and NBI is suggested, even if the most accurate result should come out from the combination of at least two out of them tests. If US or OCT is available its use could help in the lesions' volume identification, especially for bulky neoplasms that does not reach the inner cortex without any laryngeal movement impairment. The final resection margins check could be trust in CE or NBI help. For the clinical follow-up WLE and stroboscopic exams are always mandatory, together with a further second level instrumental diagnostic tool, which usually counts CAT and/or CT/PET, so far. 


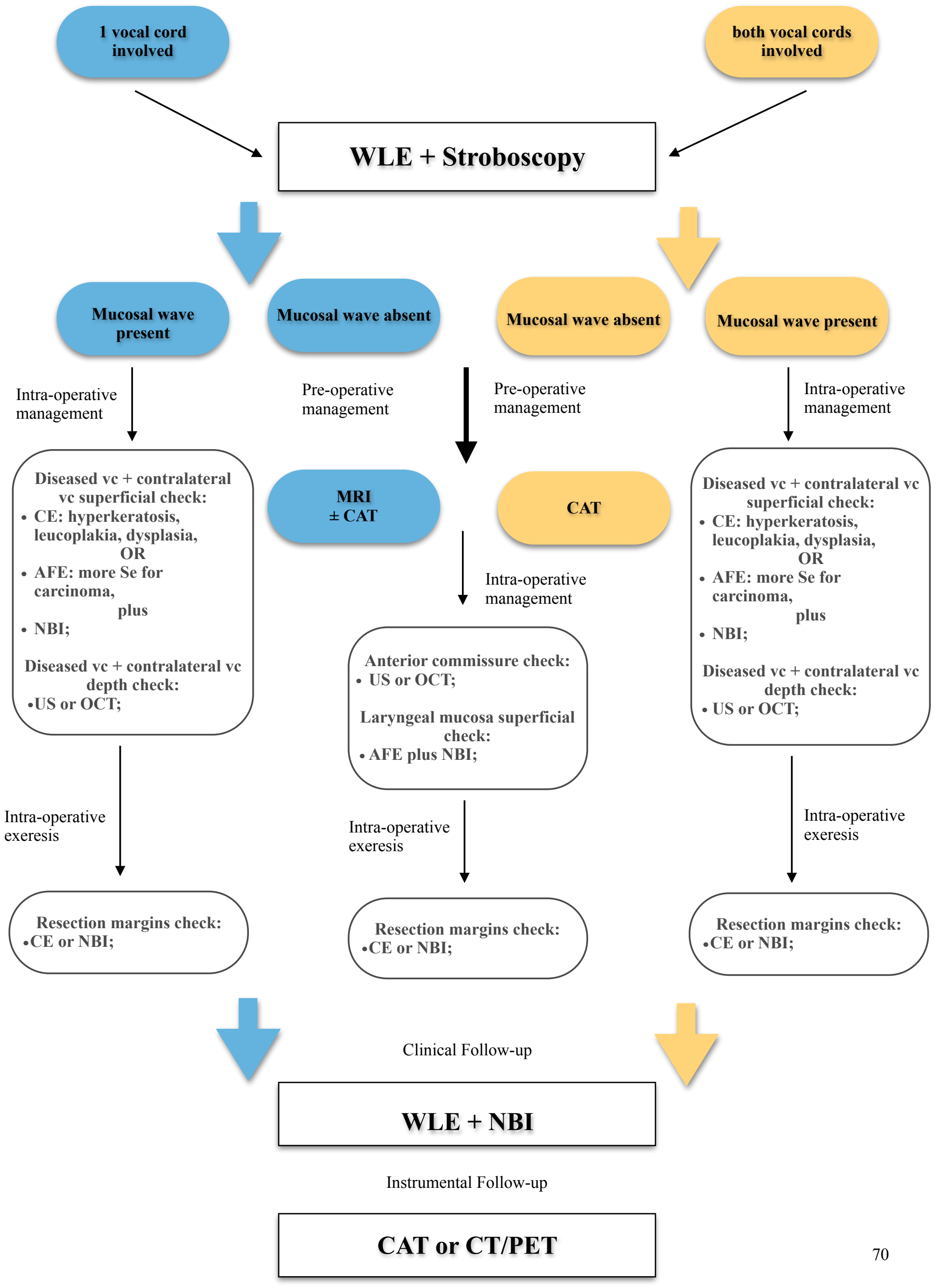




\section{Clinical implications.}

- To avoid any condition that will obstruct visualization during AFE, SAFE-3000 system and high definition white light endoscopy camera (HDTV camera) are worthy to be used. The learning curve is rather rapid, but there is the need, especially in indirect laryngoscopy, to avoid any condition in fact, anatomical sites variation, such as an omega-shaped or retrograde-positioned epiglottis could imitate AF defects at the anterior commissure and anterior third of vocal folds due to shadows from these structures. Artificial defects of the AF signal also can result from hypertrophic ventricular folds, creating shadows over the lateral part of the vocal folds.

- Compact endoscopy is the latest modification of above techniques, which combines autofluorescence and contact endoscopy together.

- The future objective should be to introduce the NBI technique into routine laryngoscopy and to use an internationally accepted endoscopic evaluation of the vascular patterns of lesions of the head and neck area. In this way, case studies can be compared based on the same classification, which can contribute to the further definition of the diagnostic validity of the technique, and to the identification of any misleading case. Despite the high benefit of NBI in distinguishing precancerous lesions from cancerous lesions, there is little benefit of NBI in identifying advanced tumors, which are readily seen in conventional WLE. Nevertheless, NBI is able to better specify their exact extension and show infiltration of neighboring structures. Additionally, NBI might detect second primaries or field cancerization in these patients (138) and it presents a potential advantage in post-treatment surveillance (247-249). Compared to AFE, NBI endoscopy has the advantage of being able to obtain the same information using both a videoendoscope coupled with a HDTV camera and rigid telescopes (137).

- Compared with CAT and MRI, US has the advantage of earlier detection of smaller tumors (T1 >3 $\mathrm{mm}$ ) as well as improvement in determining their exact size and extension at significantly lower costs. Smaller cancers (Tis, T1<3 mm) and their precursor lesions can be better assessed through optical coherence tomography (OCT), whereas advanced tumors (T2-T4) still require additional imaging techniques such as CT or MRI (250). Moreover, it permits observation of vocal cord mobility in real time $(154,160162)$. In this setting, it is a promising tool for studying the physiology of the vocal folds in phonation function. Further studies must be conducted to clarify the role of the vocal fold body in the vibration of vocal folds.

\section{Research implications.}

- Future prospective randomized clinical trials are necessary for a detailed assessment of the contribution from the most promising diagnostic tools, NBI and US, to the in vivo diagnosis of malignancies.

- In this setting, a noninvasive imaging technique that allows histologic diagnoses to be made in vivo, without taking a biopsy, will have potential significant benefits for the patient pathway. First, by focusing attention onto areas that have histologic features of malignancy this would reduce the risk of non diagnostic or unnecessary mucosal biopsies from healthy mucosa, which is particularly important for lesions on the vocal cords where preservation of the delicate superficial 
lamina propria is crucial for generating a normal voice. Second, it will potentially offer greater certainty about the position of tumor margins and facilitate decision-making during surgical tumor resection. Third, it may ultimately allow definitive histologic or molecular diagnoses to be made in the outpatient's clinic, removing the need for a general anesthesia and significantly shortening the patient's journey toward definitive treatment.

- Furthermore, primary research is required to validate other emerging techniques, such as Raman spectroscopy, and to develop their clinical applications in the larynx.

- The availability of powerful low-cost digital cameras and image-processing methods should help to standardize the analysis routines that may be used for clinical analysis. Collaboration between the clinician and the scientist in developing the software for imaging and analysis will go far in improving the knowledge base between clinical care and objective results.

- Finally, future automatic imaging analysis systems could perform the real quantification of images with diagnostic purpose. Essentially three-dimensional imaging of the superficial lamina propria is yet undeveloped. Toward that end, future evaluation about optical coherence tomography (OCT) as a new technique to evaluate the layered microstructure of the vocal fold could be implemented. OCT represents the optical analog of ultrasound and involves the detection of light backscattered off tissue boundaries (changes in refractive index). OCT has demonstrated to be potentially used intraoperatively for accurate staging of locally invasive laryngeal tumors, thanks to its spatial resolution of $<10 \mu \mathrm{m}$ (112). On the wake of promising OCT's results, the future deliver of an "optical biopsy" of the mucosa will improve the reach of a intraoperative diagnosis. Thus, OCT could be added to standard SML, allowing, in some instances, to remove the need for taking a tissue biopsy, maybe by replacing frozen section, and to guide surgeons towards more significant and representative areas of the suspect lesion. Moreover, OCT might become helpful during trans oral laser surgery since it provides a guide in tumor resection margins in three dimensions.

- Another important objective to be covered is the statistical analysis of the biomechanical parameters extracted from a large population database of voice samples, including different pathological cases as well as normal ones, taking into account side conditions such as sex, age, and habits.

\section{Disclosure}

None to declare

\section{Conflict of interest statement.}

Author declares that there is no conflict of interest, financial of otherwise.

\section{Acknowledgement.}

This manuscript is approved by the author, who has written the work, and the supervisor who has participated in correcting this work. Further, both assure that manuscript has not been published nor is under approving by other journals or editors. 


\section{References.}

1. Mehta DD, Hillman RE. Voice assessment: Updates on perceptual, acoustic, aerodynamic, and endoscopic imaging methods. Current Opinion in Otolaryngology and Head and Neck Surgery 2008;16:211-215;

2. Deliyski DD, Hillman RE. State of the art laryngeal imaging: research and clinical implications. Curr Opinion Otolaryngolg Head Neck Surg 2010;18:147-152;

3. Alberti PW. The history of laryngology: a centennial celebration. Otolaryngol Head Neck Surg 1996;114:345-354;

4. Mafee MF, Valvassori GE, Becker M. Imaging of the neck and head, 2nd edn. 2005 Thieme, Stuttgart;

5. Uloza V, Saferis V, Uloziene I. Perceptual and acoustic assessment of voice pathology and the efficacy of endolaryngeal phonomicrosurgery. J Voice 2005;19:138-145;

6. Gallivan KH, Gallivan GJ. Bilateral mixed laryngoceles: simultaneous strobovideolaryngoscopy and external video examination. J Voice 2002;16:258-266;

7. Rumboldt Z, Gordon L, Ackermann RBS. Imaging in head and neck cancer. Curr Treat Options Oncol 2006; 7:23-34;

8. Hoorweg JJ, Kruijt RH, Heijboer RJ, Eijkemans MJ, Kerrebijn JD. Reliability of interpretation of CT examination of the larynx in patients with glottic laryngeal carcinoma. Arch Otolaryngol Head Neck Surg 2006;135:129-134;

9. Rubin JS, Lee S, McGuinness J, Hore I, Hill D, Berger L. The potential role of ultrasound in differentiating solid and cystic swellings of the true vocal fold. J Voice 2004;18:231-235;

10. Schade G, Kothe C, Leuwer R. Sonography of the larynx - an alternative to laryngoscopy? HNO 2003;51:585-590;

11. Deliyski DD. Clinical Feasibility of High-Speed Videoendoscopy. Perspectives on Voice and Voice Disorders 2007;17:12-16;

12. Doellinger M. The next step in voice assessment: High-speed digital endoscopy and objective evaluation. Current Bioinformatics 2009;4:101-111;

13. Verikas A, Uloza V, Bacauskiene M, Gelzinis A, Kelertas E. Advances in laryngeal imaging. European Archives of Oto-Rhino-Laryngology 2009;266:1509-1520;

14. Oxman AD, Cook DJ, Guyatt GH. Users' guides to the medical literature. VI. How to use an overview. Evidence-Based Medicine Working Group. JAMA 1994;272:1367-71;

15. Swingler GH, Volmink J, Ioannidis JP. Number of published systematic reviews and global burden of disease: Database analysis. BMJ 2003;327:1083-4;

16. Thomas L, Wilson JA. Systematic reviews: triumph of form over substance? Clin Otolaryngol 2006;31(6):492-5;

17. Harvey RJ. Systematic review as a primer rather than endpoint for clinical research: the training perspective. Otolaryngology Head Neck Surg 2007;137:S66-S68;

18. Sacks HS, Berrier J, Reitman D, Ancona-Berk VA, Chalmers TC. Meta-analysis of randomized controlled trials. New Engl J Med 1987;316:450-5;

19. Mulrow CD. The medical review article: State of the science. Ann Intern Med 1987;106:485-8; 
20. Moher D, Cook DJ, Eastwood S, Olkin I, Rennie D, et al. for the QUOROM group. Improving the quality of reporting of meta-analysis of randomized controlled trials: The QUOROM statement. Lancet 1994;354:1896-900;

21. Callaham ML, Weber E, Young G, Wears R, Barton C. Time to publication of studies was not affected by whether results were positive. BMJ 1998;16:1536;

22. Sterne JA, Gavaghan D, Egger M. Publication and related bias in meta-analysis: power of statistical tests and prevalence in the literature. J Clin Epidemiol 2000;53:1119-29;

23. Guyatt GH, Oxman AD, Vist GE, Kunz R, Falck-Ytter Y, et al. for the GRADE Working Group. GRADE: An emerging consensus on rating quality of evidence and strength of recommendations. BMJ 2008;336:924-926;

24. Schunemann HJ, Jaeschke R, Cook DJ, Bria WF, El-Solh AA, et al. for the ATS Documents Development and Implementation Committee. An official ATS statement: Grading the quality of evidence and strength of recommendations in ATS guidelines and recommendations. Am J Respir Crit Care Med 2006;174:605-614;

25. Dickersin K. Publication bias: Recognizing the problem, understanding its origins and scope, and preventing harm. In: Rothstein HR, Sutton AJ, Borenstein M, editors. Publication bias in meta-analysis Prevention, assessment and adjustments. Chichester (UK): John Wiley \& Sons; 2005. p. 11-33;

26. Chan AW, Hrobjartsson A, Haahr MT, Gøtzsche PC, Altman DG. Empirical evidence for selective reporting of outcomes in randomized trials: Comparison of protocols to published articles. JAMA 2004;291:2457-65;

27. Chan AW, Krleza-Jeric K, Schmid I, Altman DG. Outcome reporting bias in randomized trials funded by the Canadian Institutes of Health Research. CMAJ 2004;171:735-40;

28. Higgins JPT, Altman DG, Gøtzsche PC, Jüni P, Moher D, Oxman AD, et al. The Cochrane Collaboration's tool for assessing risk of bias in randomised trials. BMJ 2011;343:d5928;

29. Viswanathan M, Ansari MT, Berkman ND, Chang S, Hartling L, McPheeters M, et al. Assessing the risk of bias of individual studies in systematic reviews of health care interventions. AHRQ Methods for Effective Health Care 2012 Mar 08;

30. Hartling L, Hamm MP, Milne A, Vandermeer B, Santaguida PL, Ansari M, et al. Testing 162 the risk of bias tool showed low reliability between individual reviewers and across consensus assessments of reviewer pairs. J Clin Epidemiol 2013;66:973-81;

31. Jadad AR, Moore RA, Carroll D, Jenkinson C, Reynolds DJ, Gavaghan DJ, et al. Assessing the quality of reports of randomized clinical trials: Is blinding necessary? Control Clin Trials 1996; 17:1-12;

32. Berlin JA. Does blinding of readers affect the results of meta-analyses? Lancet 1997;350:185-6;

33. Moja LP, Telaro E, D'Amico R, Moschetti I, Coe L, Liberati A. Assessment of methodological quality of primary studies by systematic reviews: Results of the meta-quality cross sectional study. BMJ 2005;330:1053; 
34. Sanderson S, Tatt ID, Higgins JPT. Tools for assessing quality and susceptibility to bias in observational studies in epidemiology: A systematic review and annotated bibliography. Int $\mathbf{J}$ Epidemiol 2007;36:666-76;

35. Dechartres, A. Charles, P. Hopewell, S. Ravaud, P. Altman, D.G. Reviews assessing the quality or the reporting of randomized controlled trials are increasing over time but raised questions about how quality is assessed. J Clin Epidemiol 2011;64:136-44;

36. Jüni P, Witschi A, Bloch R, Egger M. The hazards of scoring the quality of clinical trials for meta-analysis. JAMA 1999;282:1054-60;

37. Goodman S, Dickersin K. Metabias: A challenge for comparative effectiveness research. Ann Intern Med 2011;155:61-2;

38. Dickersin K. The existence of publication bias and risk factors for its occurrence. JAMA 1990;263:1385-9.; Begg CB. Publication bias: A problem in interpreting medical data. J R Stat Soc A 1988;151:419-63;

39. Dwan K, Gamble C, Williamson PR, Kirkham JJ. Systematic review of the empirical evidence of study publication bias and outcome reporting bias-An updated review. PloS One 2013;8:e66844;

40. Song F, Parekh S, Hooper L, Loke Y, Ryder J, Sutton A, et al. Dissemination and publication of research findings: an updated review of related biases . Prepress Projects, 2010;

41. Hopewell S, Loudon K, Clarke MJ, Oxman AD, Dickersin K. Publication bias in clinical trials due to statistical significance or direction of trial results. Cochrane Database Syst Rev 2009; (1):MR000006;

42. Dickersin K, Chalmers I. Recognizing, investigating and dealing with incomplete and biased reporting of clinical research: from Francis Bacon to the WHO. J R Soc Med 2011;104:532-8;

43. Williamson PR, Gamble C, Altman DG, Hutton JL. Outcome selection bias in meta-analysis. Stat Methods Med Res 2005;14:515-24;

44. Dwan K, Altman DG, Cresswell L, Blundell M, Gamble CL, Williamson PR. Comparison of protocols and registry entries to published reports for randomized controlled trials. Cochrane Database Syst Rev 2011;(1):MR000031;

45. Sterne JAC, Sutton AJ, Ioannidis JPA, Terrin N, Jones DR, Lau J, et al. Recommendations for examining and interpreting funnel plot asymmetry in meta-analyses of randomized controlled trials. BMJ 2011;343:302;

46. Dwan K, Gamble C, Kolamunnage-Dona R, Mohammed S, Powell C, Williamson P. 150 Assessing the potential for outcome reporting bias in a review: A tutorial. Trials 2010;11:52;

47. Kirkham JJ, Dwan KM, Altman DG, Gamble C, Dodd S, Smyth R, et al. The impact of outcome reporting bias in randomized controlled trials on a cohort of systematic reviews. BMJ 2010;340:c365;

48. Moher D, Tetzlaff J, Tricco AC, Sampson M, Altman DG. Epidemiology and reporting characteristics of systematic reviews. PLoS Med 2007;4:e78; 
49. Moher D, Liberati A, Tetzlaff J, Altman DG, The PRISMA Group. Methods of systematic review and meta-analysis. Preferred reporting items for systematic reviews and meta-analyses: The PRISMA Statement. J Clin Epidemiol 2009:62:1006-1012;

50. Abaid LN, Grimes DA, Schulz KF. Reducing publication bias of prospective clinical trials through trial registration. Contraception 2007;76:339-341;

51. Shamseer L, Moher D, Clarke M, Ghersi D, Liberati A et al. Preferred reporting items for systematc review and meta-analysis protocols (PRISMA-P) 2015: elaboration and explanation. BMJ 2014;349:g7647;

52. Crissman JD, Zarbo RJ. Dysplasia, in situ carcinoma, and progression to invasive squamous cell carcinoma of the upper aerodigestive tract. Am J Surg Pathol 13 Suppl 1989;1:5-16;

53. Bouquot JE, Kurland LT, Weiland LH. Laryngeal keratosis and carcinoma in the Rochester, MN, population 1935- 1984. Cancer Detect Prev 1991;15:83-91;

54. Kambic V, Gale N. Epithelial hyperplastic lesions of the larynx. Elsevier: Amsterdam,1995;

55. Fiorella R, Di Nicola V, Resta L. Epidemiological and clinical relief on hyperplastic lesions of the larynx. Acta Otolaryngol Suppl 1997;527:77-81;

56. Blackwell KE, Calcaterra TC, Fu YS. Laryngeal dysplasia: epidemiology and treatment outcome. Ann Otol Rhinol Laryngol 1995;104:596-602;

57. Maier H, Dietz A, Gewelke U, Heller WD, Weidauer H. Tobacco and alco- hol and the risk of head and neck cancer. Clin Investig 1992;70:320-327;

58. Cavani A, Negri E, Franceschi S, Barbone F, La Vecchia C. Attributable risk for laryngeal cancer in northern Italy. Cancer Epidemiol Biomarkers Prev 1994;3:121-125;

59. Kambic V, Radsel Z, Prezelj J, Zargi M. The role of testosterone in laryngeal carcinogenesis. Am J Otolaryngol 1984;5:344-349;

60. Maier H, Tisch M. Epidemiology of laryngeal cancer: results of the Heidelberg case-control study. Acta Otolaryngol Suppl 1997;527:160-164;

61. Parnes SM. Asbestos and cancer of the larynx: is there a relation- ship? Laryngoscope 1990;100:254-261;

62. Smith EM, Summersgill KF, Allen J, Hoffman HT, McCulloch T, Turek LP, Haugen TH. Human papillomavirus and risk of laryngeal cancer. Ann Otol Rhinol Laryngol 2000;109:1069-1076;

63. Kleinsasser O. Tumors of the Larynx and Hypopharynx. Georg Thieme Pub: Stuttgart,1988;

64. Wenig BM. Squamous cell carcinoma of the upper aerodigestive tract: precursors and problematic variants. Mod Pathol 2002;15:229-254;

65. Crissman JD, Visscher DW, Sakr W. Premalignant lesions of the upper aerodigestive tract: pathologic classification. J Cell Biochem Suppl 1993;17F: 49-56;

66. Hellquist H, Cardesa A, Gale N, Kambic V, Michaels L. Criteria for grading in the Ljubljana classification of epithelial hyperplastic laryngeal lesions. A study by members of the Working Group on Epithelial Hyperplastic Laryngeal Lesions of the European Society of Pathology. Histopathology 1999;34: 226-233; 
67. Barnes C, Sexton M, Sizeland A, Tiedemann K, Berkowitz RG, Waters K. Laryngo-pharyngeal carcinoma in childhood. Int J Pediatr Otorhinolaryngol 2001;61:83-86;

68. Ohlms LA, McGill T, Healy GB. Malignant laryngeal tumors in children: a 15-year experience with four patients. Ann Otol Rhinol Laryngol 1994;103:686-692;

69. Cattaruzza MS, Maisonneuve P, Boyle P. Epidemiology of laryngeal cancer. Eur J Cancer B Oral Oncol 1996;32B:293-305;

70. Rafferty MA, Fenton JE, Jones A. The history, aetiology and epidemiology of laryngeal carcinoma. Clin Otolaryngol 2001;26:442-446;

71. DeRienzo DP, Greenberg SD, Fraire AE. Carcinoma of the larynx. Changing incidence in women. Arch Otolaryngol Head Neck Surg 1991;117:681-684;

72. Patrikiou A, Sepheriadou- Mavropoulou T, Zambelis G. Bilateral traumatic bone cyst of the mandible. A case report. Oral Surg Oral Med Oral Pathol 1981;51:131-133;

73. Patternson K, Kapur S, Chandra RS. "Nasal gliomas" and related brain heteropias: a pathologist's perspective. Pediatr Pathol 1986;5:353-362;

74. Parkin DM, Whelan SL, Ferlay J, Teppo L, Thomas DB. Cancer Incidence in Five Continents, Vol. VIII. IARC 2001;Press: Lyon;

75. Olsen J, Sabreo S, Fasting U. Interaction of alcohol and tobacco as risk factors in cancer of the laryngeal region. J Epidemiol Community Health 1985;39:165-168;

76. Tuyns AJ, Esteve J, Raymond L, Berrino F, Benhamou E, Blanchet F, Boffetta P, Crosignani P, del Moral A, Lehmann W, Merletti F, Pequignot G, Riboli E, Sancho-Garnier H, Terracini B, Zubiri A, Zubiri L. Cancer of the larynx/hypopharynx, tobacco and alcohol: IARC international case-control study in Turin and Varese (Italy), Zaragoza and Navarra (Spain), Geneva (Switzerland) and Calvados (France). Int J Cancer 1988;41:483-491;

77. Sancho-Garnier H, Theobald S. Black (air-cured) and blond (flue- cured) tobacco and cancer risk II: Pharynx and larynx cancer. Eur J Cancer 1993;29A:273- 276;

78. Hedberg K, Vaughan TL, White E, Davis S, Thomas DB. Alcoholism and cancer of the larynx: a case-control study in western Washington (United States). Cancer Causes Control 1994;5:3-8;

79. Bosetti C, Talamini R, Levi F, Negri E, Franceschi S, Airoldi L, La Vecchia C. Fried foods: a risk factor for laryngeal cancer? Br J Cancer 2002;87:1230-1233;

80. Browne K, Gee JB. Asbestos exposure and laryngeal cancer. Ann Occup Hyg 2000;44:239-250;

81. Notani PN, Jayant K. Role of diet in upper aerodigestive tract cancers. Nutr Cancer 1987;10:103-113;

82. World Cancer Research Fund (WCRF). Lung. In: Food, Nutrition and the Prevention of Cancer: A Global Perspective (Part II, Cancers, nutrition and food), Food, Nutrition and the Prevention of Cancer: A Global Perspective (Part II, Cancers, nutrition and food), American Institute for Cancer research: Washington, D.C. 1997;pp. 130-147;

83. Ward PH, Hanson DG. Reflux as an etiological factor of carcinoma of the laryngopharynx. Laryngoscope 1998;98:1195-1199; Morrison MD. Is chronic gastroesophageal reflux a causative factor in glottic carcinoma? Otolaryngol Head Neck Surg 1988;99:370-373; 
84. Freije JE, Beatty TW, Campbell BH, Woodson BT, Schultz CJ, Toohill RJ. Carcinoma of the larynx in patients with gastroesophageal reflux. Am J Otolaryngol 1996;17:386-390;

85. Lichtenstein L, Jaffee HL. Chondrosarcoma of Bone. Am J Pathol 1943;553-589;

86. Paul BC, Chen S, Sridharan S, Fang Y, Amin MR, Branski RC. Diagnostic accuracy of history, laryngoscopy, and stroboscopy. Laryngoscope 2013; 123:215-219;

87. Ferlito A, Doglioni C, Rinaldo A, Devaney KO, What is the earliest non-invasive malignant lesion of the larynx? ORL J Otorhinolaryngol Relat Spec. 2000;62(2):57-59;

88. Oertel M. Das laryngo-stroboskop und die Laryngo-Stro-boskpische Untersuchung. Archiv Laryngologie Rhinologie. 1895;3:1-16;

89. Zeitels SM, Franco RA Jr, Dailey SH, Burns JA, Hillman RE, Anderson RR. Office-based treatment of glottal dysplasia and papillomatosis with the $585-\mathrm{nm}$ pulsed dye laser and local anesthesia. Ann Otol Rhinol Laryngol. 2004;113:265-276;

90. Krausert CR, Olszewski AE, Taylor LN, McMurray JS, Dailey SH, Jiang JJ. Mucosal wave measurement and visualization techniques. J Voice 2011;25:395-405;

91. Gugatschka M, Kiesler K, Beham A, Rechenmacher J, Friedrich G. Hyperplastic epithelial lesions of the vocal folds: combined use of exfoliative cytology and laryngostroboscopy in differential diagnosis. Eur Arch Otorhinolaryngol 2008;265:797-801;

92. Hirano M. Bless DM. Videostroboscopic examination of the larynx. San Diego, CA: Singular publishing group inc;1993;

93. Kendall, KA.; Leonard, R., editors. Laryngeal evaluation: Indirect laryngoscopy to high-speed digital imaging. New York, NY: Thieme Medical Publishers, Inc; 2010. (This comprehensive, full-color reference provides a thorough overview of the most recent advances in endoscopic laryngeal imaging technology combined with the information needed to interpret findings and successfully manage patients with voice disorders);

94. Moore P, von Leden H. Dynamic variation of the vibratory pattern in the normal larynx. Folia Phoniatr (Basel) 1958;10:205-238;

95. Timcke R, von Leden H, Moore P. Laryngeal vibrations: measurements of the glottic wave. Part 1. The normal vibratory cycle. Arch Otolaryngol. 1958;68:1-19;

96. Timcke R, von Leden H, Moore P. Laryngeal vibrations: II. Physiologic vibrations. Arch Otolaryngol. 1959;69:438-444; von Leden HV, Moore P,

97. Timcke R. Laryngeal vibrations: III. The patho- logic larynx. Arch Otol, Rhinol Laryngol. 1960;71:1232-1250;

98. Deguchi S, Ishimaru Y, Washio S. Preliminary evaluation of stroboscopy system using multiple light sources for obser- vation of pathological vocal fold oscillatory pattern. Ann Otol Rhinol Laryngol 2007;116:687-694;

99. Woo P. Objective measure of laryngeal imaging: what have we learned since Dr. Paul Moore. J Voice 2014; 28:69-81;

100.Dailey SH, Spanou K, Zeitels SM. The evaluation of benign glottic lesions: rigid telescopic stroboscope versus suspension microlaryngoscopy. J Voice 2007;21:112-118; 
101.Hamou JE. Microendoscopy and contact endoscopy. Brevet Francais 79, 04168 Paris, 1979. International patent PCT/FR 80/0024 Paris, 1980. US patent 4,385,810, Washington, DC;

102.Puxeddu R, Sionis S, Gerosa C, Carta F. Enhanced contact endoscopy for the detection of neoangiogenesis in tumors of the larynx and hypopharynx. Laryngoscope 2015 [Epub ahead of print];

103.Andrea M, Dias O, Santos A. Contact endoscopy during microlaryngeal surgery. A new technique for endoscopic examination of the larynx. Ann Otol Rhinol Laryngol 1995;104:3339;

104.Andrea M, Dias O, Santos A. Contact endoscopy of the vocal cord. Normal and pathology patterns. Acta Otolaryngol (Stockh) 1995;115:314-16;

105.Arens C, Glanz H, Dreyer T, Malzahn K. Compact endoscopy of the larynx. Ann Otol Rhinol Laryngol 2003;112: 113-119;

106. Warnecke A, Averbeck T, Leinung M, Soudah B, Wenzel G, Kreipe HH, Lenarz T, Stover T. Contact endoscopy for the evaluation of the pharyngeal and laryngeal mucosa. Laryngoscope 2010;120:253-258;

107.Cikojevic D, Gluncic I, Pesutic-Pisac V. Comparison of contact endoscopy and frozen section histopathology in the intra-operative diagnosis of laryngeal pathology. J Laryngol Otol 2008;122:836-839;

108.Cikojevic' D. Contact Endoscopy in the Intraoperative Diagnosis of Laryngeal Pathology. Doctoral Dissertation [in Croatian]. Zagreb: School of Medicine, University of Zagreb, 2005;60-3;

109. Wardrop PJ, Sim S, McLaren K. Contact endoscopy of the larynx: a quantitative study. J Laryngol Otol 2000;114:437-40;

110.Carriero E, Galli J, Fadda G, Di Girolamo S, Ottaviani F, Paludetti G. Preliminary experiences with contact endoscopy of the larynx. Eur Arch Otorhinolaryngol 2000;257: 68-71;

111.Ledda GP, Puxeddu R. Carbon dioxide laser microsurgery for early glottic carcinoma. Otolaryngol Head Neck Surg 2006;134:911-915;

112.Hughes OR, Stone N, Kraft M, Arns C, Birchall MA. Optical and molecular techniques to identify tumor margins within the larynx. Head Neck 2010;32:1544-1553;

113.Richards-Kortum R, Sevick-Muraca E. Quantitative optical spectroscopy for tissue diagnosis. Annu Rev Phys Chem 1996;47:555-606;

114.Schwartz JP, Passonneau JV, Johnson GS, Pastan I. The effect of growth conditions on NADp and NADH concentrations and the NADp:NADH ratio in normal and transformed fibroblasts. J Biol Chem 1974;249:4138-4143;

115.Gillenwater A, Jacob R, Richards-Kortum R. Fluorescence spectroscopy: a technique with potential to improve the early detection of aerodigestive tract neoplasia. Head Neck 1998;20:556-562;

116.Malzahn K, Dreyer T, Glanz H, Arens C. Autofluorescence endoscopy in the diagnosis of early laryngeal cancer and its precursor lesions. Laryngoscope 2002;112: 488-493; 
117.Wagnieres GA, Star WM, Wilson BC. In vivo fluorescence spectroscopy and imaging for oncological applications. Photochem Photobiol 1998;68: 603-632;

118.Uppal A, Gupta PK. Measurement of NADH concentration in normal and malignant human tissues from breast and oral cavity. Biotechnol Appl Biochem 2003;37(pt 1):45-50;

119.Baletic N, Petrovic Z, Pendjer I, Malicevic H. Autofluorescent diagnostics in laryngeal pathology. Eur Arch Otorhinolaryngol 2004;261:233-237;

120.Jacobson MC, deVere White R, Demos SG. In vivo testing of a prototype system providing simultaneous white light and near infrared autofluorescence image acquisition for detection of bladder cancer. J Biomed Opt 2012;17:036011;

121.Sieron-Stoltny K, Kwiatek S, Latos W, et al. Autofluorescence endoscopy with "real-time" digital image processing in differential diagnostics of selected benign and malignant lesions in the oesophagus. Photodiagnosis Photodyn Ther 2012;9:5-10;

122.Policard A, Etude sur les aspects offerts par des tumeurs expérimentales examinées à la lumière de Wood, C R Soc Biol (Paris), 1924, 91:1423-1424;

123.Alfano R, Tata D, Cordero J, Tomashefsky P, Longo F, Alfano M, Laser induced fluorescence spectroscopy from native cancerous and normal tissue, IEEE J Quant Electron, 1984, 20(12): 1507-1511;

124.Harries ML, Lam S, MacAulay C, Qu J, Palcic B. Diagnostic imaging of the larynx: autofluorescence of laryngeal tumours using the helium-cadmium laser. J Laryngol Otol 1995;109:108-110;

125.Chissov VI, Sokolov VV, Filonenko EV, Menenkov VD, Zharkova NN, Kozlov DN. Clinical fluorescent diagnosis of tumors using photosensitizer photogem. Khirurgiia (Mosk) 1995;5:3741 ;

126.Zargi M, Fajdiga I, Smid L. Autofluorescence imaging in the diagnosis of laryngeal cancer. Eur Arch Otorhinolaryngol 2000;257:17-23;

127.Arens C, Malzahn K, Dias O, Andrea M, Glanz H. Endoscopic imaging techniques in the diagnosis of laryngeal carcinoma and its precursor lesions. Laryngorhinootologie 1999;78:685691;

128.Delank W, Khanavkar B, Nakhosteen J, Stoll W. A pilot study of autofluorescence endoscopy for the in vivo detection of laryngeal cancer. Laryngoscope 2000;110:368-373;

129.Mostafa BE, Shafik AG, Fawaz S. The role of flexible autofluorescence laryngoscopy in the diagnosis of malignant lesions of the larynx. Acta Otolaryngol 2007;127: 175-179;

130.Arens C, Reussner D, Neubacher H, Woenckhaus J, Glanz H. Spectrometric measurement in laryngeal can- cer. Eur Arch Otorhinolaryngol 2006;263:1001-1007;

131.Y. Sano, M. Kobayashi, and Y. Hamamoto. New diagnostic method based on color imaging using narrowband imaging (NBI) endoscopy system for gastrointestinal tract. Gastrointestinal Endoscopy, 2001;53:AB125;

132.Irjala H, Matar N, Remacle M, Georges L. Pharyngo-laryngeal examination with the narrow band imaging technology: early experience. Eur Arch Otorhinolaryngol 2011;268:801-806; 
133.C. Piazza, O. Dessouky, G. Peretti, D. Cocco, L. De Benedetto, and P. Nicolai. Narrow-band imaging: a new tool for evaluation of head and neck squamous cell carcinomas. Review of the literature. Acta Otorhinolaryngol Ital 2008;28:49-54;

134.Kumagai Y, Inoue H, Nagai K, Kawano T, Iwai T. Magnifying endoscopy, stereoscopic microscopy, and the microvascular architecture of superficial esophageal carcinoma. Endoscopy 2002;34:369-75;

135.Ni XG, He S, Xu ZG, et al. Endoscopic diagnosis of laryngeal cancer and precancerous lesions by narrow band imaging. J Laryngol Otol 2011; 125:288-296;

136. Watanabe A, Taniguchi M, Tsujie H, Hosokawa M, Fujita M, Sasaki S. The value of narrow band imaging for early detection of laryngeal cancer. Eur Arch Otorhinolaryngol 2009;266:1017-1023;

137.Piazza C, Cocco D, De Benedetto L, Del Bon F, Nicolai P, Peretti G. Narrow band imaging and high definition television in the assessment of laryngeal cancer: a prosepctive study on 279 paztients. Eur Arch Otorhinolaryngol 2010;267:409-414;

138. Bertino G, Cacciola S, Fernandes WB Jr, et al. Effectiveness of narrow band imaging in the detection of premalignant and malignant lesions of the larynx: validation of a new endoscopic clinical classification. Head Neck 2015. [Epub ahead of print];

139.Masaki T, Katada C, Nakayama M, Takeda M, Miyamoto S, Seino Y, Koizumi W, Tanabe S, Horiguchi S, Okamoto M. Narrow band imaging in the diagnosis of intra-epithelial and invasive laryngeal squamous cell carcinoma: a preliminary report of two cases. Auris Nasus Larynx 2009;36:712-716;

140.Piazza C, Del Bon F, Peretti G, Nicolai P. Narrow band imaging in endoscopic evaluation of the larynx. Curr Opin Otolaryngol Head Neck Surg 2012;20:472-476;

141.Tjon Pian Gi RE, Halmos GB, van Hemel BM, et al. Narrow band imaging is a new technique in visualization of recurrent respiratory papillomatosis. Laryngoscope 2012;122:1826-1830;

142.Imaizumi M, Okano W, Tada Y, Omori K. Surgical treatment of laryngeal papillomatosis using narrow band imaging. Otolaryngol Head Neck Surg 2012;147:522-524;

143. Watanabe A, Taniguchi M, Tsujie H, Hosokawa M, Fujita M, Sasaki S. The value of narrow band imaging endoscope for early head and neck cancers. Otolaryngol Head Neck Surg 2008;138:446-451;

144.Ugumori T, Muto M, Hayashi R, Hayashi T, Kishimoto S. Prospective study of early detection of pharyngeal superficial carcinoma with the narrowband imaging laryngoscope. Head Neck 2009;31:189-194;

145.Lin YC, Watanabe A, Chen WC, Lee KF, Lee IL, Wang WH. Narrowband imaging for early detection of malignant tumors and radiation effect after treatment of head and neck cancer. Arch Otolaryngol Head Neck Surg 2010;136:234-239;

146.Piazza C, Cocco D, Del Bon F, Mangili S, Nicolai P, Peretti G. Narrow band imaging and high definition television in the endoscopic evaluation of upper aero-digestive tract cancer. Acta Otorhinolaryngol Ital 2011;31:70-75; 
147.Shinozaki T, Hayashi R, Ebihara M, et al. Narrow band imaging endoscopy for unknown primary tumor sites of the neck. Head Neck 2012;34:826-829;

148.Lin YC, Wang WH, Lee KF, Tsai WC, Weng HH. Value of narrow band imaging endoscopy in early mucosal head and neck cancer. Head Neck 2012;34:1574-1579;

149.Yang SW, Lee YS, Chang LC, et al. Diagnostic significance of narrow-band imaging for detecting high-grade dysplasia, carcinoma in situ, and carcinoma in oral leukoplakia. Laryngoscope 2012;122:2754-2761;

150.Lukes P, Zabrodsky M, Lukesova E, Chovanec M, Astl J, Betka J and Plzak J. The role of NBI HDTV magnifying endoscopy in the pre histologic diagnosis of laryngeal papillomatosis and spinocellular cancer. Biomed Res Int 2014;2014:285486;

151.Ahuja AT, Ying M, Ho SY, et al. Ultrasound of malignant cervical lymph nodes. Cancer Imaging 2008; 8:48-56;

152.Chevallier P, Marcy PY, Arens C, Raffalli C, Padovani B, Bruneton JN. Larynx and Hypopharynx. In: Bruneton JN. Applications of sonography in head and neck pathology. New York: Springer 2002;165-191;

153.Kuribayashi S, Miyashita T, Nakamizo M, Yagi T, Kumita S. Utility of sonography for evaluation of clinical T1 and T2 glottic carcinoma. J Ultrasound Med 2009;28:1429-1440;

154.Desai AA, Pandya VK, Bhalani DB, Desai S, Parikh BD. Value of ultrasonography in laryngeal and laryngopharyngeal cancers. Indian J Otolaryngol Head Neck Surg 2004;56:191-195;

155.Gritzmann N, Traxler M, Grasl M, Pavelka R. Advanced laryngeal cancer: sonographic assessment. Radiology 1989;171:171-175;

156.Rubin JS, Lee S, McGuinness J, Hore I, Hill D, Berger L. The potential role of ultrasound in differentiating solid and cystic swellings of the true vocal fold. J Voice 2004;18:231-235;

157.Vats A, Worley GA, de Bruyn R, Porter H, Albert DM, Bailey CM. Laryngeal ultrasound to assess vocal fold paralysis in children. J Laryngol Otol 2004;118:429-431;

158.Ooi LL, Chan HS, Soo KC. Color Doppler imaging for vocal cord palsy. Head Neck 1995;17:20-23;

159.Loveday EJ, Bleach NR, Van Hasselt CA, Metreweli C. Ultrasound imaging in laryngeal cancer: a preliminary study. Clin Radiol 1994;49:676-682;

160.Hu Q, Luo F, Zhu SY, Zhang Z, Mao YP, Guan Hui Guan X. Staging of laryngeal carcinoma: comparison of high-frequency sonography and contrast-enhanced computed tomography. Clin Radiol 2012;67:140-147;

161.Loveday EJ. Ultrasound of the larynx. Imaging 2003;15:109-14;

162.Xia CX, Zhu Q, Zhao HX, Yan F, Li SL, Zhang SM. Usefulness of ultrasonography in assessment of laryngeal carcinoma. Br J Radiol 2013;86:20130343;

163.Hu Q, Zhu SY, Zhang Z, Luo F, Mao YP, Guan XH. Assessment of glottic squamous cell carcinoma. Copmarison of sonography and non-contrast-enhanced magnetic resonance imaging. J Ultrasound Med 2011;30:1467-1474;

164.Kraft M; Mende S, Arnoux A, Arens C. Anatomical landmarks for endosonography of the larynx. Head Neck 2010;32:326-332; 
165.Arens C, Glanz H. Endoscopic high-frequency ultrasound of the larynx. Eur Arch Otorhinolaryngol 1999;256:316-322;

166.Hsiao TY, Wang CL, Chen CN, Hsieh FJ, Shau YW. Noninvasive assessment of laryngeal phonation function using color Doppler ultrasound imaging. Ultrasound Med Biol 2001;27:1035-1040;

167.Hsiao TY, Wang CL, Chen CN, Hsieh FJ, Shau YW. Elasticity of human vocal folds measured in vivo using color Doppler imaging. Ultrasound Med Biol 2002;28:1145-1152;

168.Hirano M. Morphological structure of the vocal cord as a vibrator and its variations. Folia Phoniatr 1974;26:89-94;

169.Hirano M. Phonosurgery: Basic and clinical investigations. Otologia (Fukuoka) 1975;21(Suppl. 1):239-262;

170.Tsai CG, Chen JH, Shau YW, Hsiao TY. Dynamic B.mode ultrasound imaging of vocal fold vibration during phonation. Ultrasound Med Biol 2009;35:1812-1818;

171.Yeager VL, Lawson C, Archer CR. Ossification of the laryngeal cartilages as it relates to computed tomography. Invest Radiol 1982;17:11-19;

172.Gregor RT, Hammond K. Framework invasion by laryngeal carcinoma. Am J Surg 1987;154:452-8;

173.Gallo A, Mocetti P, De Vincentiis M, Simonelli M, Ciampini S, Bianco P et al. Neoplastic infiltration of laryngeal cartilages: histocytochemical study. Laryngoscope 1992;102:891- 5;

174.Becker M. Neoplastic invasion of laryngeal cartilage: radiologic diagnosis and therapeutic implications. Eur J Radiol 2000;33:216-29;

175.Kuno H, Onaya H, Fuji S, Ojiri H, Otani K, Satake M. Primary staging of laryngeal and hypo pharyngeal cancer: CT, MR imaging and dual-energy CT. Eur J Radiol 2014;83:e23-25;

176.Sulfaro S, Barzan L, Querin F, et al. T staging of the laryngohypopharyngeal carcinoma. A 7year multidisciplinary experience. Arch Otolaryngol Head Neck Surg 1989;115(5):613-20;

177.Zbaren P, Becker M, Lang H. Pretherapeutic staging of laryngeal carcinoma. Clinical findings, computed tomography, and magnetic resonance imaging compared with histopathology. Cancer 1996;77:1263-73;

178.Becker M, Zbaren P, Laeng H, Stoupis C, Porcellini B, Vock P. Neoplastic invasion of the laryngeal cartilage: comparison of MR imaging with CT with histopathologic correlation. Radiology 1995; 194: 661-9;

179.Becker M, Zbaren P, Delavelle J, et al. Neoplastic invasion of the laryngeal cartilage: reassessment of criteria for diagnosis at CT. Radiology 1997;203(2):521-32;

180.Beitler JJ, Muller S, Grist WJ, Corey A; Klein AM, Johns MM, Perkins CL, Davis LW, Udayasanker U, Landry JC, Shin DM, Hudguns PA. Prognostic accuracy of computed tomography findings for patients with laryngeal cancer undergoing laryngectomy. J Clin Oncol 2010;28:2318-2322;

181.Barbosa MM, Araujo Jr VJ, Boasquevisque E, et al. Anterior vocal commissure invasion in laryngeal carcinoma diagnosis. Laryngoscope 2005; 115: 724-30;

182.Hermans R. Head and neck cancer imaging. Berlin: Springer; 2006. pp 31-80; 
183.Baum U, Greess H, Lell M, Nomayr A, Lenz M. Imaging of head and neck tumors - methods: CT, spiral CT, multislice spiral CT. Eur J Radiol 2000;33:153 - 60;

184.Zbaren P, Becker M, Lang H. Pretherapeutic staging of hypopharyngeal carcinoma. Clinical findings. Computed tomography, and magnetic resonance imaging compared with histopathological evaluation. Arch Otolaryngol Head Neck Surg 1997;123:908-13;

185.Zbaren P, Becker M, Lang H. Staging of laryngeal cancer: endoscopy, computed tomography and magnetic resonance imaging versus histopathology. Eur Arch Otorhinolaryngol Suppl 1997;1:S117-22;

186.Declercq A, Van den Hauwe L, Van Marck E, Van de Heyning PH, Spanoghe M, De Schepper AM. Patterns of framework invasion in patients with laryngeal cancer: correlation of in vitro magnetic resonance imaging and pathological findings. Acta Otolaryngol 1998;118:892-5;

187.Selbie WS, Gewalt SL, Ludlow CL. Developing an anatomical model of the human laryngeal cartilages from magnetic resonance imaging. J Acoust Soc Am 2002;112:1077-1090;

188.Hu A, Wilson T, Ladak H, Haase P, Fung K. Three-dimensional educational computer model of the larynx: voicing a new direction. Arch Otolaryngol Head Neck Surg 2009;135:677-681;

189.Herrera VL, Viereck JC, Lopez-Guerra G, Kumai Y, Kobler J, Karajanagi S, Park H, Hillman R, Zeitels SM. 11.7 Tesla magnetic resonance microimaging of laryngeal tissue architecture. Laryngoscope 2009;119(11):2187-2194. (This study utilized MR microimaging of excised larynges allowing high-resolution imaging of laryngeal tissue microstructure. Histology confirmed the utility of MR microimaging of modeled scar, implant residence time, and tissue responses.);

190.Ahmad M, Dargaud J, Morin A, Cotton F. Dynamic MRI of larynx and vocal fold vibrations in normal phonation. Journal of Voice 2009;23(2):235-239. (This study used dynamic MRI of the larynx and vocal folds during phonation for measuring the vertical laryngeal movements and the glottal angle of the vocal folds opening and closing in dynamic phase.);

191.Gordin A, Daitzchman M, Dowek I, Yefremov N, Golz A, Keidar Z, Bar-Shalom R, Kuten A, Israel O. Fluorodeoxyglucose-Positron emission tomography/computed tomography imaging in patients with carcinoma of the larnx: diagnostic accuracy and impact on clinical management. Laryngoscope 2006;116:237-278;

192.Kresnik E, Mikosch P, Gallowitsch HJ, et al. Evaluation of head and neck cancer with ${ }^{18}$ FFDG PET: a comparison with conventional methods. Eur J Nucl Med 2001;28: 816-821;

193.Jeong HS, Chung MK, Beak CH, Choi JY, Son YI, Kim HJ, Hong SD, Bok KH. Combined 18F-FDG PET/CT imaging for the initial evaluation of glottic cancer. Clin Exp Otorhinolaryngol 2008;1:35-40;

194.Reitsma JB, Glas AS, Rutjes AW, et al. Bivariate analysis of sensitivity and specificity produces informative summary measures in diagnostic reviews. J Clin Epidemiol 2005;58:982-990;

195.Higgins JP, Thompson SG, Deeks JJ, Altman DG. Measuring inconsistency in meta-analysis. BMJ 2003;327:557-560; 
196.Caffier PP, Schmidt B, Gross M, Karnetzky K, Nawka T, Rotter A, Seipelt M, Sedlmaier B. A comparison of white light laryngostroboscopy versus autofluorescence endoscopy in the evaluation of vocal fold patholohy. Laryngoscope 2013;123:1729-1734;

197.Arens C, Glanz H, Wonckhaus J, Hersemeyer K, Kraft M. Histologic assessment of epithelial thickness in early laryngeal cancer or precursor lesions and its impact on endoscopic imaging. Eur Arch Otorhinolaryngol 2007;264:645-649;

198.Kraft M, Betz CS, Leunig A, Arens C. Value of fluorescence endoscopy for the early diagnosis of laryngeal cancer and its precursor lesions. Head Neck 2011;33:941-948;

199.Crosetti E, Pilolli F, Succo G. A new strategy for endoscopic staging of laryngeal carcinoma: multistep endoscopy. Acta Otorhinolaryngl Ital 2012;32:175-181;

200.Uloza V, Vegiene A, Pribuisiene R, Saferis V. Quantitative evaluation of video laryngostroboscopy: reliability of the basic parameters. J Voice 2013;27:361-368;

201.Succo G, Garofalo P, Fantini M, Monticone V, Abbona GC, Crosetti E. Direct autofluorescence during $\mathrm{CO} 2$ laser surgery of the larynx: can it really help the surgeon? Acta Otorhinolaryngol Ital 2014;34:174-183;

202.Lucioni M, Bertolin A, D’Ascanio L, et al. Margin photocoagulation in laser surgery for early glottic cancer: impact on disease local control. Otolaryngol Head Neck Surg 2012;146:600-5;

203.Baletic N, Malicevic H, Petrovic Z, Marinkovic-Eric J, Peric A. Advantages and limitations of the autofluorescent diagnostics of the laryngeal cancer and precancerosis. Eur Arch Otorhinolaryngol 2010;267:925-931;

204.Rydell R, Eker C, Andersson_engels S, Krogdahi A, Wahlberg P, Svanberg K. Fluorescence investigations to classify malignant laryngeal lesions in vivo. Head Neck 2008;30:419-426;

205.Saetti R, Derosas F, Silvestrini M, Narne S. Efficacy of autofluorescence videoendoscopy in the diagnosis of laryngeal lesions. Acta Otolaryngol Ital 2007;27:181-185;

206.Kraft M, Fostiropoulos K, Gurtler N, Arnoux A, Davaris N, Arens C. Value of narrow band imaging in the early diagnosis of laryngeal cancer. Head Neck 2014; [Epub ahead of print];

207.Arens C, Reussner D, Woenkhaus J, Leunig A, Betz CS, Glanz H. Inderect fluorescence laryngoscopy in the diagnosis of precancerous and cancerous laryngeal lesions. Eur Arch Otorhinolaryngol 2007;264:621-626;

208.Zabrodsky M, Lukes P, Lukesova E, Boucek J, Plaz J. The role of narrow band imaging in the detection of recurrent laryngeal and hypo pharyngeal cancer after curative radiotherapy. Biomed Res Int 2014;2014:175398. doi: 10.1155/2014/175398. Epub 2014 Jun 30;

209.Kraft M, Bruns N, Hugens-Penzel M, Arens C. Clinicl value of endosonography in the assessment of laryngeal cancer. Head Neck 2013;35:195-200;

210.Zech M, Scheer M, Maier H, Heppt W. Endosonography of the larynx. Eur Arch Otorhinolaryngol 1994;251:480-481;

211.Arens C, Eistert B, Glanz H, Waas W. Endolaryngeal high-frequency ultrasound. Eur Arch Otorhinolaryngol 1998;255:250-255;

212.Tamura E, Kitahara S, Kohno N. Clinical assessment of intralaryngeal ultrasonography. Laryngoscope 2001;111:1767-1770; 
213. Tamura E, Kitahara S, Kohno N. Intralaryngeal application of a miniaturized ultrasonic probe. Acta Otolaryngol 2002;122:92-95;

214.Tsui PH, Huang CC, Sun L, Dailey SH, Shung KK. Med Phys 2011;38:2019-2026;

215.Castelijns JA, Gerritsen GJ, Kaiser MC, Valk J, van Zanten TE, Golding RG et al. Invasion of laryngeal cartilage by cancer: comparison of CT and MR imaging. Radiology 1988;167:199206;

216.Sulfaro S, Querin F, Barzan L, Lutman M, Comoretto R, Volpe R et al. Pathologic assessment of computerized tomography accuracy for the evaluation of the laryngeal cartilaginous framework in laryngopharyngeal carcinomas. Tumori 1989;75:156-62;

217.Amilibia E, Juan A, Nogues J, Manos M, Monfort JL, Dicenta M. Neoplastic invasion of laryngeal cartilage: diagnosis by computed tomography [in Spanish]. Acta Otorrinolaringol Esp 2001;52:207-10;

218.Allegra E, Ferrise P, trapasso S, Trapuzzano O, Barca A, Tamburrini S, Garozzo A. Early glottic cancer: role of MRI in the preoperative staging. Biomed Res Int 2014;2014:890385;

219.Han MW, Kim SA, Cho KJ, Lee JH, Lim HK, Roh JL, Choi SH, Kim SY, Nam SY. Diagnostic accuracy of computed tomography findings for patients undegoing salvage total laryngectomy. Acta Otolaryngol 2013;133:620-625;

220.Hartl DM, Landry G, Bidault F, Hans S, Julieron M, Mamelle G, Janot F, Brasnu DF. CT-scan prediction of thyroid cartilage invasion for early laryngeal squamous cell carcinoma. Eur Arch otorhinolaryngol 2013;270:287-291;

221.Celebi I, Basak M; Ucgul A, Yildirim H, Oz A, Vural C. Functional imaging of larynx via 256slice multi-detector computed tomography in patients with laryngeal tumors: a faster, better and more reliable pre-therapeutic evaluation. Eur J Radiol 2012;81:e541-547;

222.Beser M, Gultekin E, Yener M, Zeybek ME, Oner B, Topcu V. Detection of laryngeal tumors and tumoral extension by multislice computed tomography virtual laryngoscopy (MSCT-VL). Eur Arch Otorhinolaryngol 2009;266:1953-1958;

223.Fernandes R, Gopalan P, Spyridakou C, Joseph G, Kumar M. Predictive indicators for thyroid cartilage involvement in carcinoma of the larynx seen on spiral computed tomography scans. JLO 2006;120:857-860;

224.Banko B, Djukic V, Milovanovic J, Kovac J, Novakovic Z, Maksimovic R. MRI in evaluation of neoplastic invasion into preepiglottic and paraglottic space. Auris Nasus Larynx 2014;41:471-474;

225.Shang DS, Ruan LX, Zhou SH, Bao YY, Cheng KJ, Wang QY. Differentiating laryngeal carcinomas from precursor lesions by diffusion-weighted magnetic resonance at $3.0 \mathrm{~T}$ : a preliminary study. PLoS One 2013;8:e68622;

226.Kinshuck AJ, Goodyear PW, Lancaster J, Roland NJ, Jackson S, Hanlon R, Lewi-Jones H, Sheard J, Jones TM. Accuracy of magnetic resonance imaging in diagnosing thyroid cartilage and thyroid gland invasion by squamous cell carcinoma in laryngectomy patients. J Laryngol Otol 2012;126:302-306; 
227.Banko B, Djukic V, Milovanovic J, Kovac J, Artiko V, Maksimovic R. Diagnostic significance of magnetic resonance imaging in preoperative evaluation of patients with laryngeal tumors. Eau Arch Otorhinolaryngol 2011;268:1617-23;

228.Lim JY, Park IS, Park SW, Kim JW, Kim YM. Potential pitfalls and therapeutic implications of pretherapeutic radiologic staging in glottic cancer. Acta Otolaryngol 2011;131:869-875;

229.Becker M, Zbaren P, Casselman JW, Kohler R, Dulduerov P, Becker CD. Neoplastic invasion of laryngeal cartilage: reassessment of criteria for diagnosis at MR imaging. Radiology 2008;249:255;

230.Cohen SM, Thomas S, Roy N, Kim J, Courey M. Frequency and factors associated with use of videolaryngostroboscopy in voice disorders assessment. Laryngoscope 2014;124:2118-2124;

231.Djukic V, Milovanovic J, Jotic AD, Vukasinovic M. Stroboscopy in detection of laryngeal dysplasia effectiveness and limitations. J Voice 2014;28:262.e13-262.e21;

232.Rosen CA. Stroboscopy as a research instrument: development of a perceptual evaluation tool. Laryngoscope. 2005;115:423-428;

233.Peretti G, Piazza C, Berlucchi M, Cappiello J, Giudice M, Nicolai P. Pre- and intraoperative assessment of midcord erythroleukoplakias: a prospective study on 52 patients. Eur Arch Otorhinolaryngol. 2003;260:525-528;

234.Arens C, Dreyer T, Glanz H, Malzahn K. Indirect auto- fluorescence laryngoscopy in the diagnosis of laryngeal cancer and its precursor lesions. Eur Arch Otorhinolaryngol 2004;261:71-76;

235.Wang TD, van Dam J, Crawford JM, Preisinger EA, Wang Y, Feld M. Fluorescence endoscopic imaging of human colonic adenomas. Gastroenterology 1996;111:1182-1191;

236.Gottschalk W. Ein Messverfahren zur Bestimmung der optischen Parameter biologischer Gewebe in vitro, Inaugural Dissertation, Universität Karlsruhe;1993;

237.Dobre M, Poenaru M, Balica NC, Doros CI. Detection of early laryngeal cancer and its precursor lesions by real-time autofluorescence imaging system. Rom J Morphol Embryol 2014;55:1377-1381;

238.Raap M, Koop N, Brandt R, Birngruber R, Hüttmann G, Schönwi- eler R (2003) Quantitative AutoXuoreszenzlaryngoscopie zur Frühkennung maligner Stimmlippenveränderungen. Wissenschaftlichen Jahrestagung der Deutsche Geselschaft für Phoniatrie und Pädaudiologie, Rostock, Poster Presentation;

239. Muto M, Sano Y, Fuji S, Ochiai A, Yoshida S. endoscopic diagnosis of intraepithelial squamous neoplasia in head and neck esophageal mucosal sites. Dig Endoscop 2006;18:2-5;

240.Kara MA, Freme PP, Fockens P, Kate FW, Bergman PD. Endoscopic video-autofluorescence imaging followed by narrow band imaging for detecting early neoplasia in Barrett's esophagus. Gastrointest Endosc 2006;64:176-178;

241.Bozzato A, Zenk J, Gottwald F, Koch M, Iro H. Influence of thyroid cartilage ossification in laryngeal ultrasound [in German]. Laryngorhinootologie 2007;86:276-281;

242.Erkan M, Tolu I, Aslan T, Güney E. Ultrasonography in laryngeal cancers. J Laryngol Otol 1993;107:65-68; 
243.Rothberg R, Noyek AM, Freeman JL, Steinhardt ML, Stoll S, Goldfinger M. Thyroid cartilage imaging with diagnostic ultrasound: correlative studies. Arch Otolaryngol Head Neck Surg 1986;112:503-515;

244.Ljumanović R, Langendijk JA, Hoekstra OS, Knol DL, Leemans CR, Castelijns JA. Pre- and post-radiotherapy MRI results as a predictive model for response in laryngeal carcinoma. Eur Radiol 2008; 18:2231-2240;

245.Connor S. Laryngeal cancer: how does the radiologist help? Cancer Imaging 2007;7:93-103;

246.Gilbert K, Dalley RW, Maronian N, et al. Staging of laryngeal cancer using 64-channel multidetector row CT: comparison of standard neck CT with dedicated breath-maneuver laryngeal CT. AJNR Am J Neuroradiol 2010;31:251-6;

247.Muto M, Nakane M, Katada C, et al. Squamous cell carcinoma in situ at oropharyngeal and hypopharyngeal mucosal sites. Cancer 2004;101:1375-81;

248.Lin YC, Wang WH. Narrow-band imaging for detecting early recurrent nasopharyngeal carcinoma. head neck 2011;33:591-4;

249.Piazza C, Cocco D, de Benedetto L, et al. Role of narrow-band imaging and high-definition television in the surveillance of head and neck squamous cell cancer after chemo- and/or radiotherapy. Eur Arch Otorhinolaryngol 2010;267:1423-8;

250.Kraft M, Glanz H, Wisweh H, Lubatschowski H, Arens C. Clinical value of optical coherence tomography in laryngology. Head Neck 2008;30: 1628-1635. 